\title{
(1)
}

UNIVERSIDAD PERUANA DE CIENCIAS APLICADAS

FACULTAD DE INGENIERÍA

CARRERA DE INGENIERÍA CIVIL

\section{PROPUESTA DE MEJORA DEL NIVEL DE \\ SERVICIO MEDIANTE LA REVERSIBILIDAD DEL SENTIDO DE TRÁNSITO EN HORA PICO EN LA \\ PANAMERICANA NORTE ENTRE LA AVENIDA \\ TOMAS VALLE Y EL PUENTE PEATONAL CONTROL ZARUMILLA}

\author{
Tesis \\ Para optar el título de Ingeniero Civil
}

AUTOR:

JESUS CRISTIAN BAUTISTA ÑAUPARI (0000-0003-4132-8347)

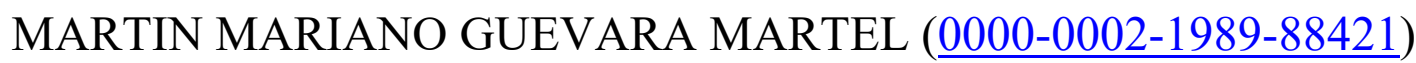

ASESOR:

ING. ALDO RAFAEL BRAVO LIZANO (ㅁ000-0003-4835-795X) 


\section{Jesus Bautista Ñaupari}

El presente trabajo es un esfuerzo mutuo entre mis familiares $y$ amistades: Mi familia que siempre estuvo apoyándome en cada paso que doy y mis compañeros que siempre creyeron en mis capacidades.

\section{Martín Guevara Martel}

El agradecimiento de este proyecto va dirigido primero a Dios ya que sin la bendición y su amor todo hubiera sido un total fracaso, también para mí docente Abel Bravo que gracias a su conocimiento y ayuda pude concluir con éxito este proyecto, a mis padres Maria y César y hermanos Francisco, Marco y Paulo, que estuvieron siempre apoyándome en mis metas. 


\section{RESUMEN}

La presente investigación ligada a la ingeniería de transportes pretende aplicar la gestión de carril reversible, en una determinada hora, en el tramo norte - sur de la Panamericana Norte entre la avenida Tomas Valle y el puente peatonal Control Zarumilla, esto ubicado en el distrito de San Martín de Porras. Esta aplicación de reversibilidad puede generar de manera positiva el tránsito de dicho caso de estudio. Se sabe que San Martín de Porres es uno de los distritos más afectados por el tráfico vehicular y la Panamericana Norte es uno de sus puntos más críticos en lo que se refiere a congestión. Por eso, través de un estudio de estados de tráfico a nivel mesoscópico y con la ayuda del HCM 2010 y el Software Aimsun verificaremos los niveles de servicios actuales y los de la gestión de reversibilidad del caso de estudio para así compararlos y ver las consecuencias favorables que tiene el implementar un carril adicional al tramo más congestionado.

Palabras clave: Niveles de servicio, carril reversible, hora pico, grado de saturación, microscópico, mesoscópico, macroscópico, flujos ininterrumpidos, autopista básica, velocidad de flujo libre, holgura lateral, aforo vehicular. 


\begin{abstract}
The present investigation linked to the engineering of transports intends to apply the management of reversible lane, in a determined hour, in the north - south section of the Panamericana Norte between the avenue Tomas Valle and the pedestrian control Zarumilla bridge, this located in the district of San Martín de Porras This application of reversibility can positively generate the transit of said case study. It is known that San Martin de Porres is one of the districts most affected by vehicular traffic and the PanAmerican Highway is one of its most critical points when it comes to congestion. Therefore, through a study of traffic states at the mesoscopic level and with the help of the Highway Capacity Manual (HCM 2010) and the Aimsun Software, we will verify the current service levels and the reversibility management of the case study. compare them and see the favorable consequences of implementing an additional lane to the most congested section.
\end{abstract}

Key words: Service levels, reversible lane, peak hour, degree of saturation, microscopic, mesoscopic, macroscopic, uninterrupted flows, basic highway, free flow velocity, lateral clearance, vehicular capacity. 


\section{INDICE DE CONTENIDO}

RESUMEN .................................................................................................... III

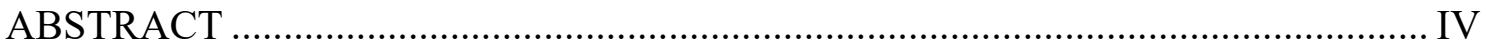

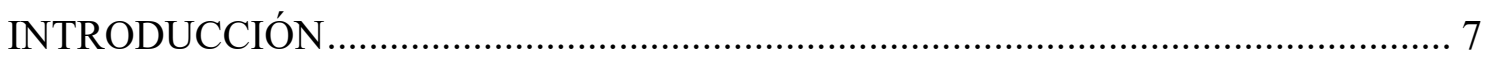

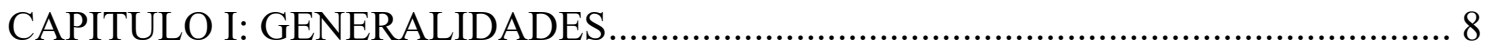

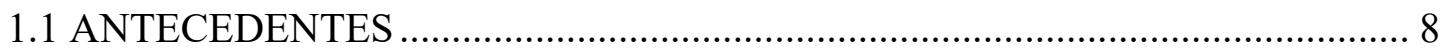

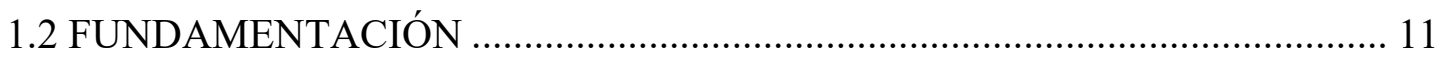

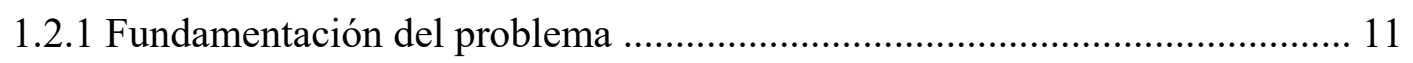

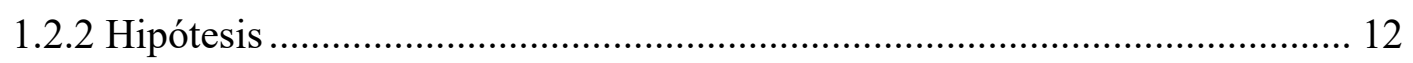

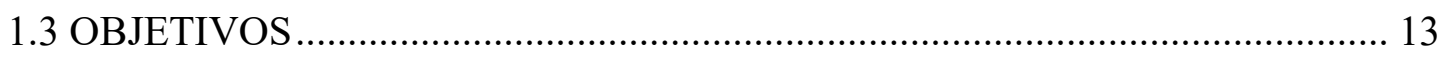

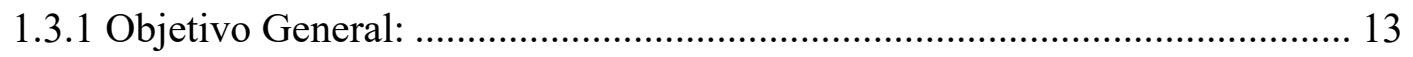

1.3.2 Objetivos Específicos e Indicadores de logro ........................................... 14

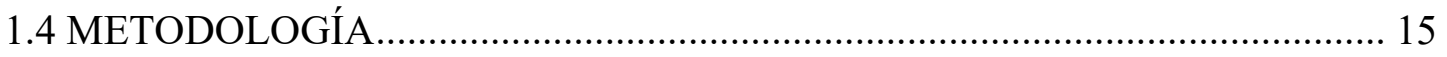

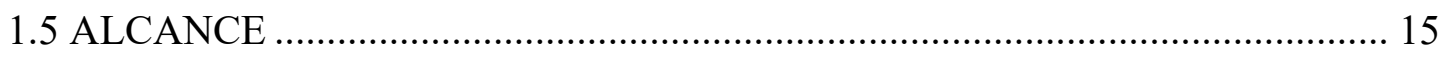

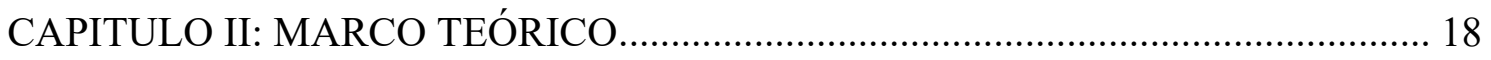

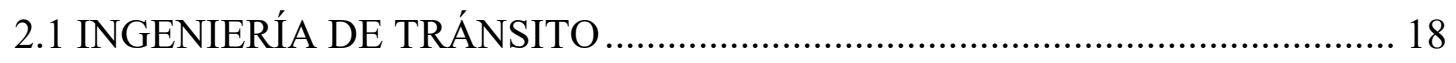

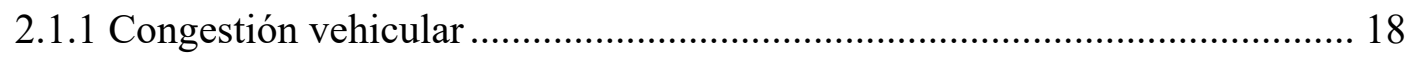

2.1.2 Tipos de Modelación del tráfico................................................................ 19

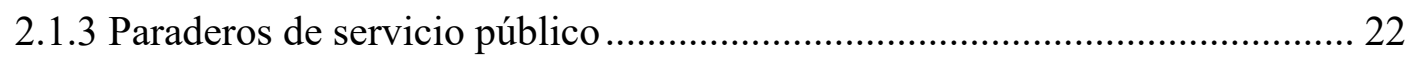

2.2 CONCEPTO DEL MANUAL HCM 2010 ......................................................... 22

2.2.1 Guía de Uso del Manual HCM 2010 ........................................................... 22

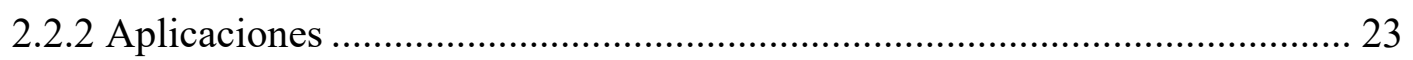

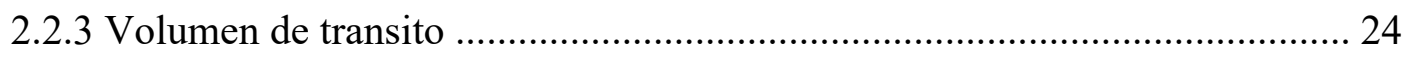

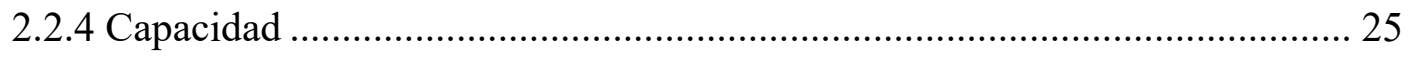

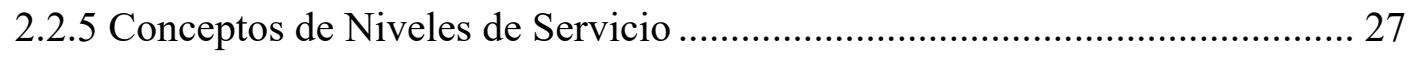

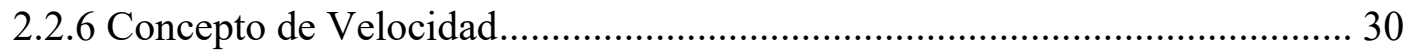

2.3 FLUJOS ININTERRUMPIDOS PARA AUTOPISTAS DE SEGMENTOS

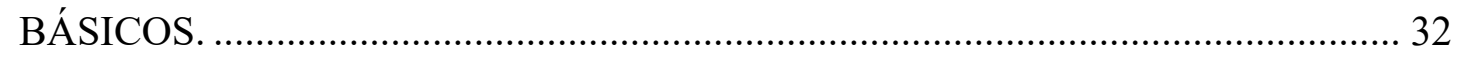

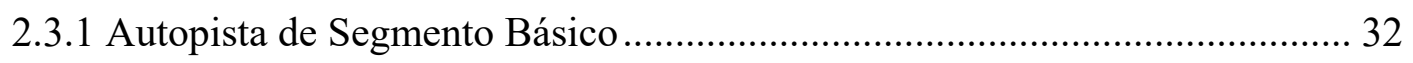

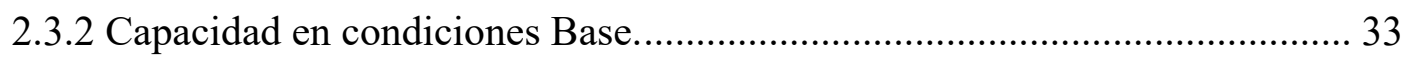

2.3.3 Niveles de servicio para Autopistas de segmentos Básicos ......................... 34 


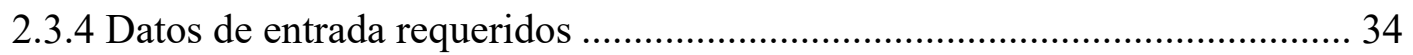

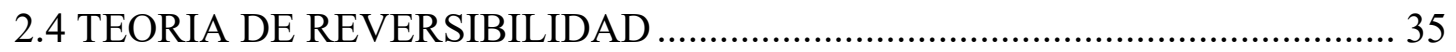

2.5 TEORIA DE AFOROS VEHICULARES......................................................... 36

2.6 FACTORES DE CONVERSIÓN A UNIDAD DE COCHE PATRÓN (UCP)... 37

2.7 DISPOSITIVOS DE CONTROL VEHICULAR ............................................... 38

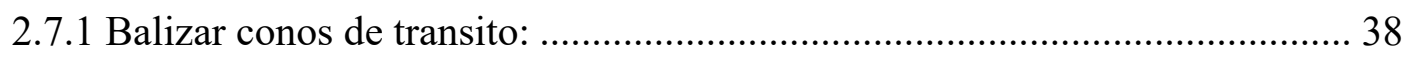

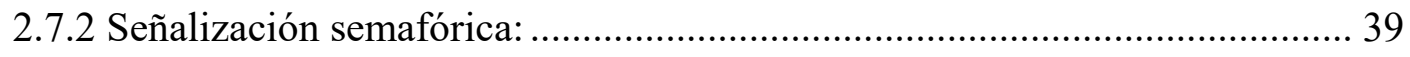

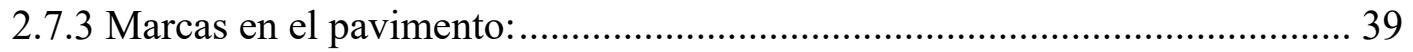

2.7.4 Pantallas de mensajes variables:.............................................................. 41

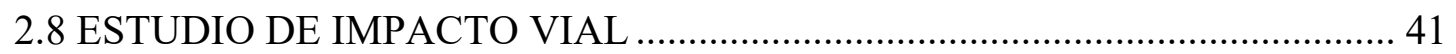

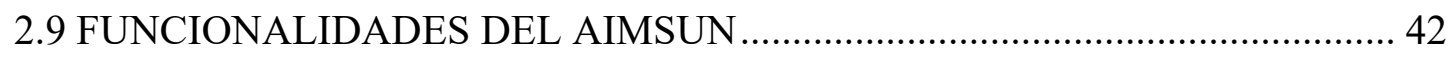

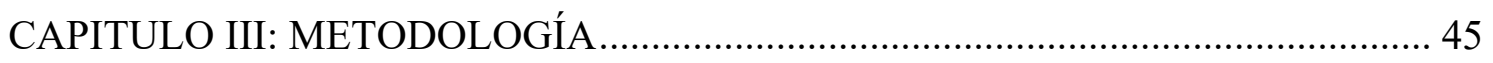

3.1 AFOROS VEHICULARES PARA EL CASO DE ESTUDIO ............................. 45

3.2 SEGMENTO DE AUTOPISTA BÁSICA …..................................................... 46

3.2.1 Descripción y procedimiento de la metodología ........................................... 47

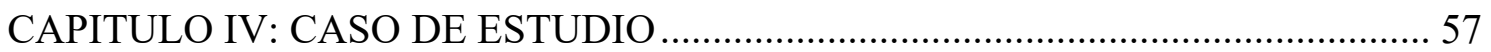

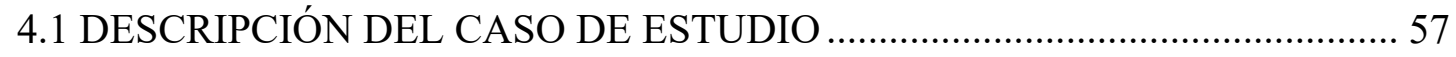

4.2 CLASIFICACIÓN DEL SEGMENTO DE AUTOPISTA EN CASO DE ESTUDIO 58

4.3 AFORO VEHICULARES DEL CASO DE ESTUDIO ....................................... 60

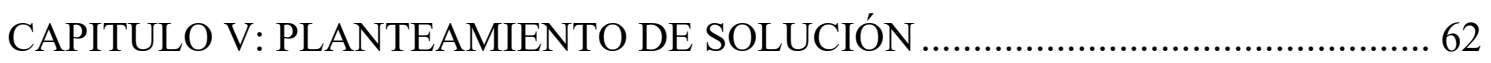

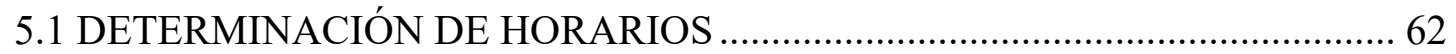

5.2 PROTOCOLO DE IMPLEMENTACIÓN DE LA GESTIÓN DEL CARRIL

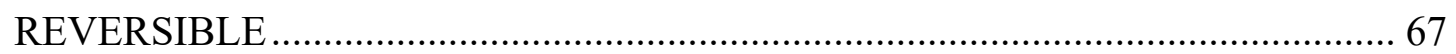

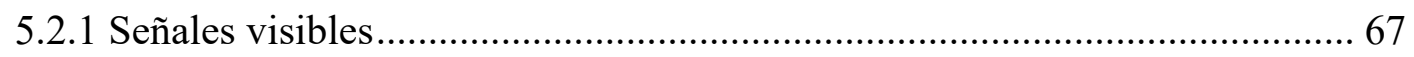

5.3 ANALISIS DE PROYECCIÓ FUTURA DEL TRANSITO. .............................. 71

5.4 PLANTEAMIENTO DE NIVELES DE SERVICIO_........................................ 72

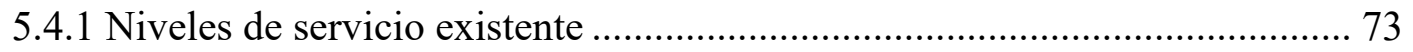

5.4.2 Niveles de servicio planteando la solución.................................................... 75

5.5 ANÁLISIS DEL PROCESAMIENTO DE DATOS …....................................... 78

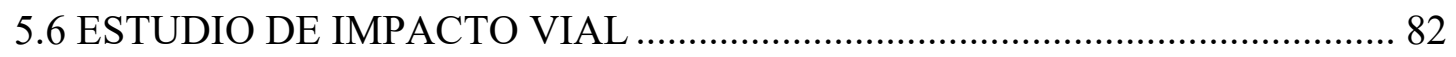

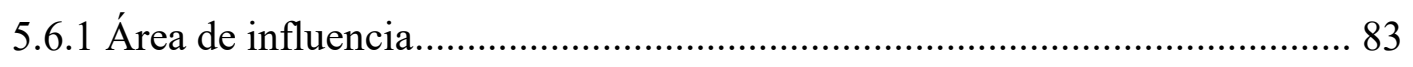

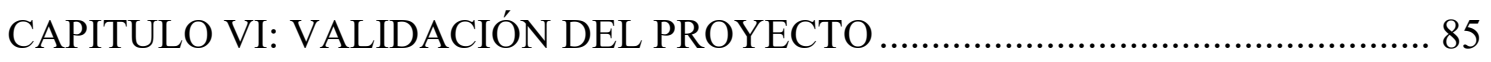

6.1 CREACIÓN DE LA RED VIAL DEL CASO DE ESTUDIO............................. 85 
6.1.1 Importar base cartográfica desde Open Street Maps .................................. 85

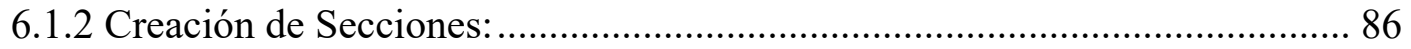

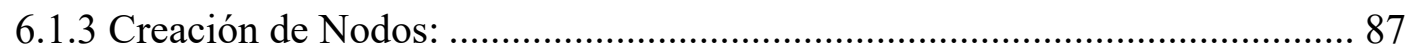

6.1.4 Jerarquización Vial, ancho de carriles, número de carriles y velocidades: ... 88

6.2 ASIGNACIÓN DEL TRAFICO .................................................................. 90

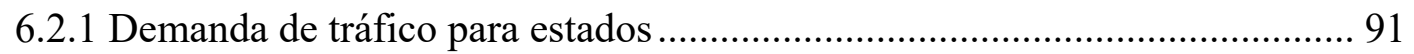

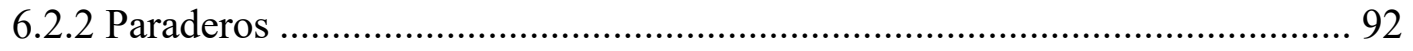

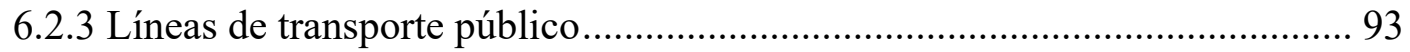

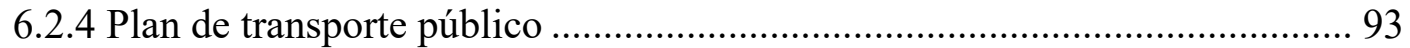

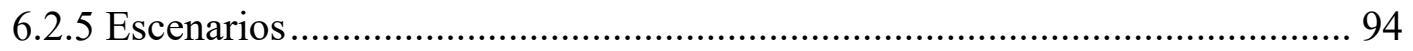

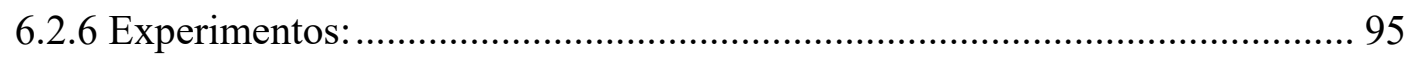

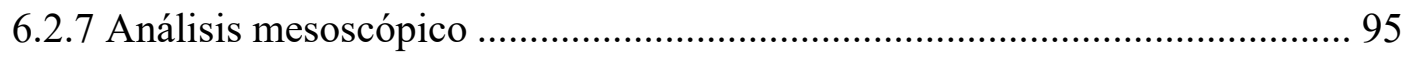

6.2.8 Número de Replicaciones ........................................................................ 96

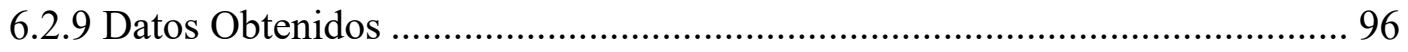

6.3 ANALISIS Y COMPARACION DE LOS RESULTADOS CON LOS DATOS DE

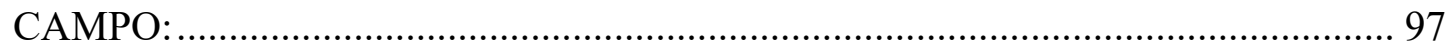

6.4 CALIBRACION DEL MODELO CON DATOS DE CAMPO: ......................... 100

CAPITULO VII: CONCLUSIONES Y RECOMENDACIONES.............................. 103

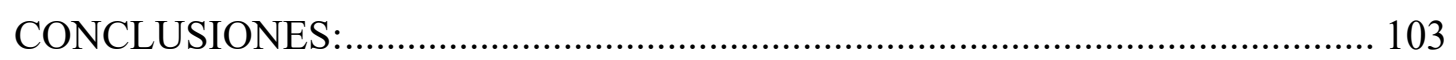

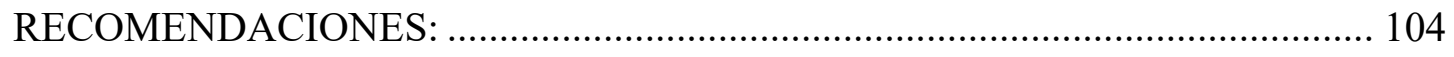

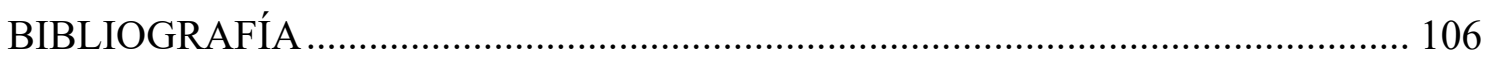




\section{INDICE DE IMÁGENES}

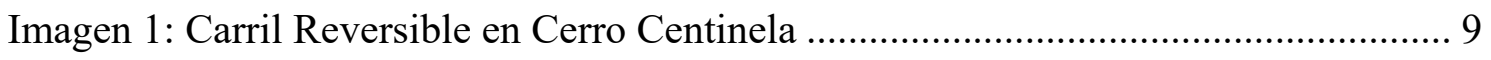

Imagen 2: Plan Verano "Cambio de Sentido" ............................................................. 10

Imagen 3: Diseño de la zona de trabajo en la primera fase de construcción................... 10

Imagen 4: Cuello de Botella de 6 a 3 carriles, cerca de la municipalidad SMP............. 12

Imagen 5: Ilustración de los carriles de ida y regreso de Norte y Sur............................ 13

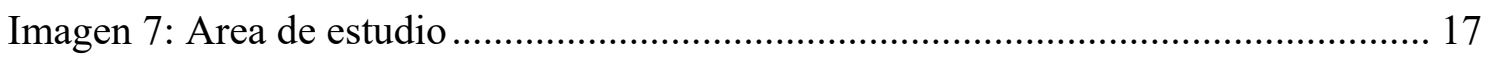

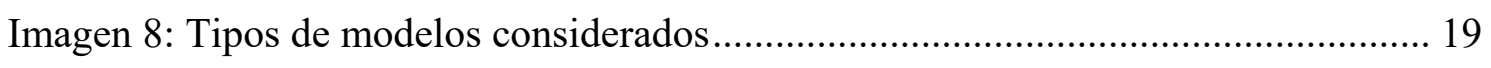

Imagen 9: Principales estrategias de simulación del tráfico....................................... 21

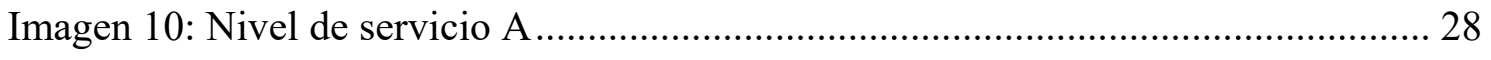

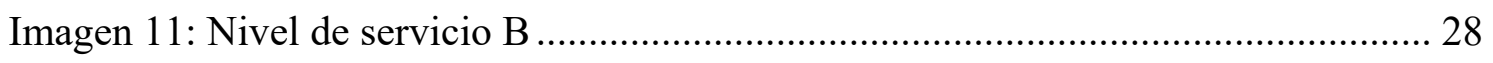

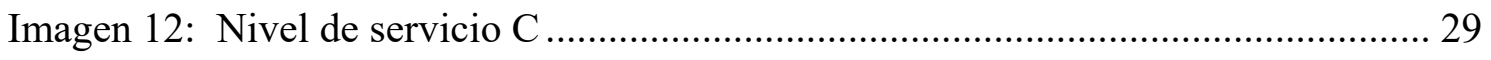

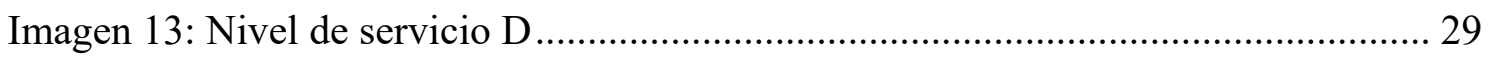

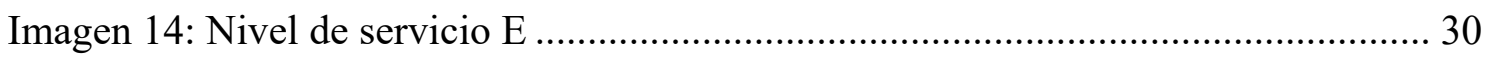

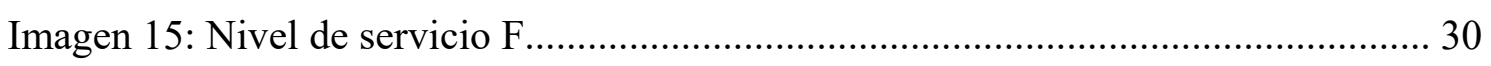

Imagen 16: Grafico Velocidad de Flujo Libre vs Capacidad ...................................... 33

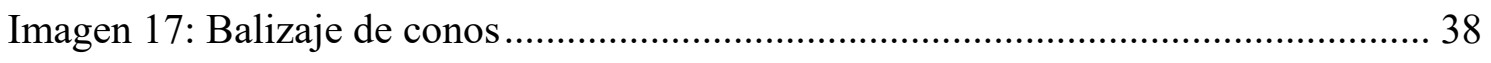

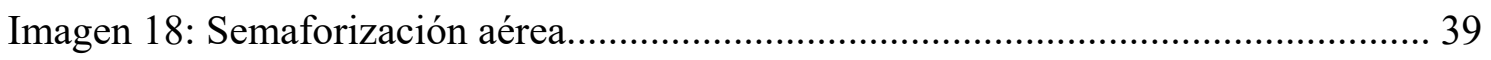

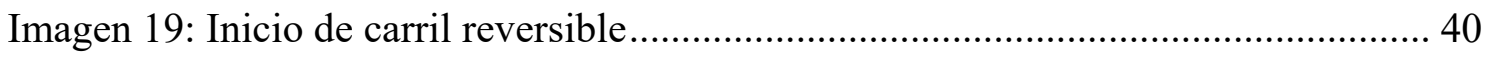

Imagen 20: Tramo de transición de carril reversible ....................................................... 40

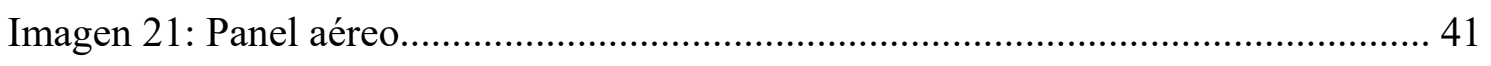

Imagen 22: Flujograma del Segmento de Autopista Básica........................................ 47

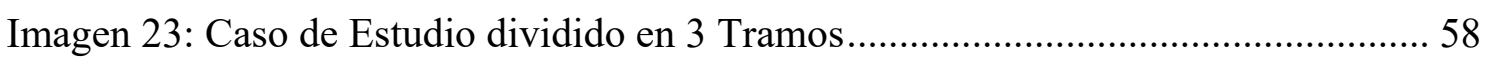

Imagen 24: Segmento A - Intercambio Vial Tomas Valle .......................................... 59

Imagen 25: Segmento B - Intercambio Vial Eduardo de Habich .................................. 59

Imagen 26: Sección C - Segmento de Autopista Básica ............................................... 59

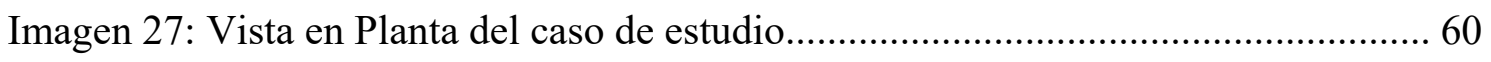

Imagen 28: Puntos de aforos vehiculares (Fuente: Red Vial del distrito de SMP) ........ 61

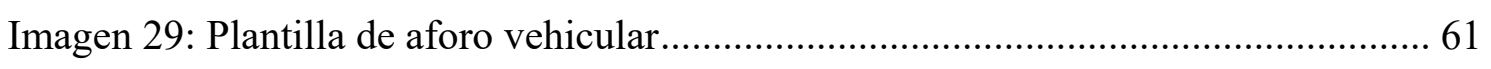

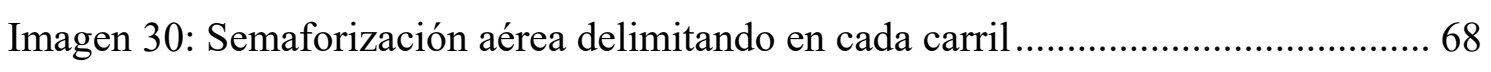

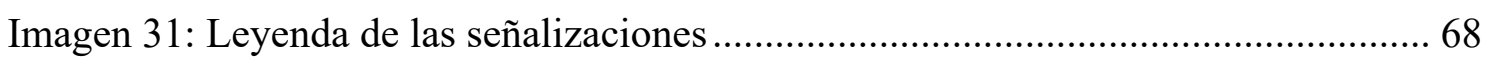


Imagen 32: Marca en el pavimento en la entrada y salida de carril reversible. 69

Imagen 33: Referencia de señales preventivas....

Imagen 34: Delimitacion de conos en la vía reversible ................................................... 70

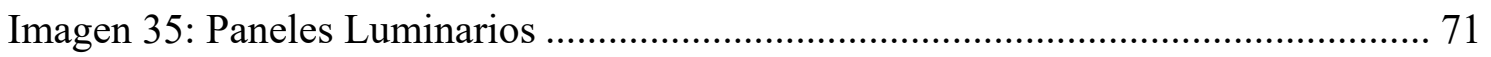

Imagen 36: Proyección del Parque Automotor ...................................................... 72

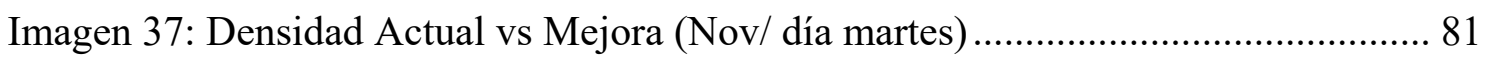

Imagen 38: Densidad Actual vs Mejora (Nov/ día jueves) ............................................ 81

Imagen 39: Densidad Mejorada vs Densidad Proyectada (Nov/ día martes) ................. 81

Imagen 40: Densidad Mejorada vs Densidad Proyectada (Nov/ día jueves) .................. 82

Imagen 41: Rutas alternas a la vía donde se aplicará la reversibilidad de carril ............ 84

Imagen 42: Red Vial del distrito de San Martín de Porres en OSM .............................. 86

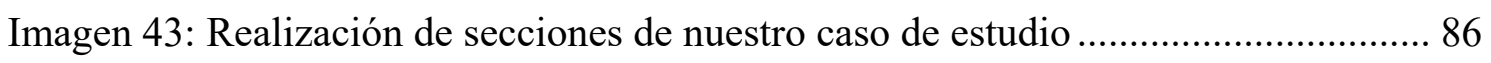

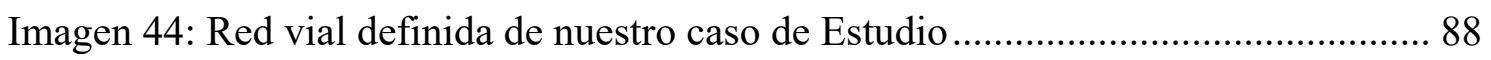

Imagen 45: Definiendo jerarquías de vía en nuestro caso de estudio............................. 89

Imagen 46: Definiendo ancho de carril en las vías de nuestro caso de estudio............... 89

Imagen 47: Definiendo velocidades a las vías de nuestro caso de estudio..................... 90

Imagen 48: Estado del tráfico de acuerdo al tipo de vehículo...................................... 91

Imagen 49: Demanda de tráfico para nuestro caso de estudio ...................................... 92

Imagen 50: Ubicación de paraderos del transporte público en nuestro caso de estudio 93

Imagen 51: modelación de las líneas de transporte público ......................................... 93

Imagen 52: Generar Plan de Transporte Público............................................................ 94

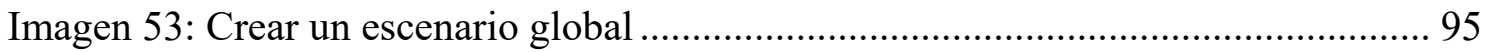

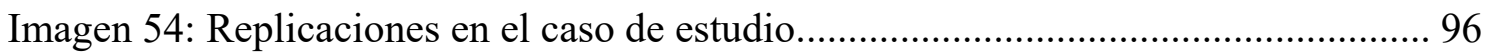

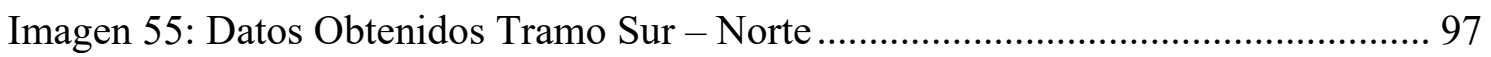

Imagen 56: Datos obtenidos en el Tramo Norte - Sur ............................................... 97

Imagen 57: Tramo Eduardo de Habich, diseñado con la gestión de reversibilidad ........ 98

Imagen 58: Tramo cerca de Tomas Valle aplicando el diseño de reversibilidad........... 99

Imagen 59: Tramo Norte - Sur con gestión de reversibilidad........................................ 99

Imagen 60: Tramo Sur - Norte con gestión de reversibilidad...................................... 100

Imagen 61: Datos de Flujograma en un punto de aforo (Pte, Control Zarumilla)........ 101

Imagen 62: Resultados de Campo (Pte, Control Zarumilla) ....................................... 101

Imagen 63: Comparativo de datos (Pte, Control Zarumilla) ....................................... 102 


\section{INDICE DE TABLAS}

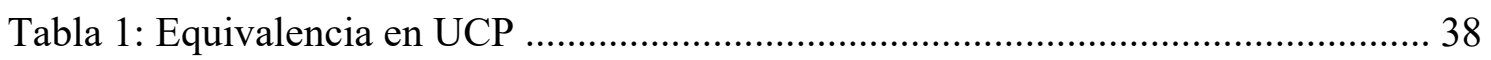

Tabla 2: Ajuste de ancho de carril en relación al FFS .................................................. 49

Tabla 3: Adaptación del FFS debido a la obstrucción del carril .................................... 50

Tabla 4: PCE para camiones, autobuses y vehículos recreativos ................................. 52

Tabla 5: Ajuste de volumen de la demanda para vehículos pesados............................. 53

Tabla 6: Ajuste de volumen de la demanda para vehículos RVs .................................. 54

Tabla 7: Valores de los ET para diversas situaciones. ................................................ 54

Tabla 8: Niveles de servicio con respecto a la densidad ............................................. 56

Tabla 9: Resumen del día martes del mes de noviembre del 2016............................... 63

Tabla 10: Resumen del día jueves del mes de noviembre del 2016 .............................. 63

Tabla 11: Resumen del día martes del mes de febrero del 2017................................... 64

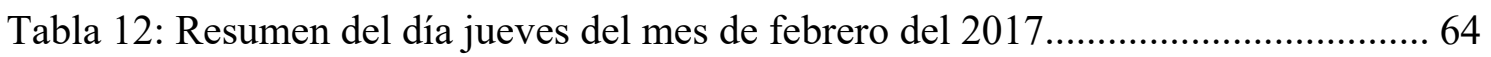

Tabla 13: Resumen del día martes del mes de noviembre del 2016............................. 65

Tabla 14: Resumen del día jueves del mes de noviembre del 2016 .............................. 65

Tabla 15: Resumen del día martes del mes de noviembre del 2016............................ 66

Tabla 16: Resumen del día jueves del mes de noviembre del 2016 ............................. 66

Tabla 17: Parque Automotor Lima y Callao ................................................................ 72

Tabla 18:Resumen Niveles de Servicio Actual (Nov 2016)......................................... 78

Tabla 19: Resumen de Niveles de Servicio con Reversibilidad ..................................... 79

Tabla 20: Resumen de Niveles de servicio con Reversibilidad a Proyección Futura .... 79

Tabla 21: Porcentaje de incremento o decremento de la densidad del tránsito con los

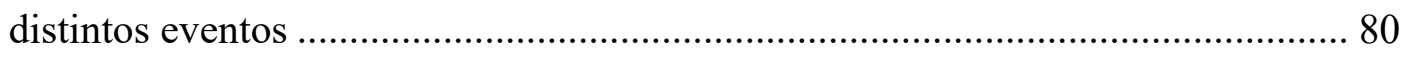




\section{INTRODUCCIÓN}

En la actualidad las grandes urbes del mundo se enfrentan diariamente al problema de movilizar a sus habitantes a sus diferentes destinos. Estos desplazamientos implican un tiempo de recorrido que se incrementa con el transcurso de los años ya que el parque automotor crece a un ritmo exponencial que se encuentra con el crecimiento y desarrollo de la infraestructura vial de las ciudades.

Si bien existen proyectos de transporte público masivo de pasajeros, estos aún no se adaptan a las necesidades y la demanda por parte de los ciudadanos como se manifiesta en caso del Metropolitano y La línea 1 del Tren Eléctrico para la ciudad del Lima-Perú.

En este proyecto de investigación se aborda la problemática de la congestión vehicular, que se puede definir como una condición de un flujo saturado con consecuencias negativas en los conductores como incrementos en los tiempos de viaje, estrés, violencia vial e incluso accidentalidad, desde un enfoque en el que se busca incentivar el uso de una mejora en la gestión vial mediante intervención y reglamentación al comportamiento de los conductores.

Si bien la congestión vehicular se debe en gran medida a la insuficiente red vial y al mal estado en que se encuentran las vías, a la mala sincronización de los semáforos, a la falta de educación vial, a los accidentes, a los vehículos con problemas mecánicos y al uso inadecuado de los carriles por parte de los automovilistas lo cual agrava la situación. Atacar dicha problemática teniendo en cuenta lo anterior puede generar alternativas como es el caso del cerro centinela, en la cual optan por un carril reversible en hora pico de la mañana.

El estudio pretende establecer, desde los fundamentos teóricos de la ingeniería de tránsito y transporte, estadística y modelación, un diseño en la vía de evitamiento, en el tramo de tomas valle hasta el puente control zarumilla, el cual está enfocada al correcto manejo de los carriles como propuesta de mejoramiento del flujo vehicular, manifestándose en la disminución del tiempo de viaje. En la investigación se hará una simulación de la situación actual de movilidad de la vía en estudio y optando por utilizar una estrategia de mejora del flujo vehicular, como lo es el carril reversible. 


\section{CAPITULO I: GENERALIDADES}

\subsection{ANTECEDENTES}

La congestión del tránsito vehicular ha ido en aumento en gran parte de la ciudad de Lima, y todo indica que seguirá agravándose, constituyendo un peligro para la calidad de vida de los limeños. Actualmente, la ciudad de Lima está tomando medidas para solucionar el problema del tráfico vehicular, tal es el caso del distrito de La Molina donde tienen como principal solución la reversibilidad del tránsito en avenidas principales a horas pico. Una de las que se implementaron fue exactamente a lo largo del Cerro Centinela de lunes a viernes, durante las 6:30am hasta las 8:30am, habilitando un tercer carril que es aprovechado por la poca utilidad de la vía aledaña como se muestra en la Imagen 1. Como resultado se da mejor fluidez vehicular a una de las dos salidas que tiene este distrito, acortando el tiempo de cruce en dicha zona. Otro caso similar de carril reversible se da en la temporada de verano a lo largo de la Panamericana Sur entre la Playa Santa María y el Puente Atocongo, como se muestra en la imagen 2, es una extensión de más de 40 kilómetros en donde se aplica la reversibilidad de carril los domingos y feriados de 4pm a $8 \mathrm{pm}$, esto con la finalidad de evitar la congestión de vehículos que retornan de las playas del sur.

Esta aplicación de reversibilidad de carril no es ajena en otros países, de hecho, nació en los Estados Unidos en los años 70, a través del Transportation Demand Management (TDM). Según el paper “Traffic Flow at a Freeway Work Zone with Reversible Median Lane”, Alemania usó esta gestión de reversibilidad en una de sus autopistas que estaba siendo refaccionada como se evidencia en la Imagen 3. A pesar de que el motivo de esta gestión fue por obras en la autopista, el objetivo fue reducir el tráfico vehicular al igual que en nuestro caso de estudio. La investigación demostró que la gestión de carril reversible fue un instrumento útil, seguro y de sistema inteligente del tráfico para zonas de trabajo en autopistas con altas fluctuaciones en hora pico.

"En la zona de trabajo analizada, se podrían ahorrar pérdidas de tiempo de viaje estimadas en 400.000 veh-hora durante dos meses de obras viales por la aplicación del carril reversible en comparación con una sola reducción de carril en una dirección.” (Helen Waleczek, Justin Geistefeldt, Dijana Cindric-Middendorf, y Gerd Riegelhuth ,2016) 
También, según el paper "Study on Flow Direction Changing Method of Reversible Lanes on Urban Arterial Roadways in China", en Beijing se aplicó esta gestión de reversibilidad en autopistas urbanas de flujos interrumpidos, que tiene intersecciones, pases peatonales y semaforizaciones. Un caso mucho más complejo que necesita la coordinación de un sistema integrado de transporte para que la reversibilidad pueda funcionar de forma segura.

Cabe resaltar que la propuesta de la reversibilidad del tránsito en avenidas principales y autopistas es una solución que se adapta al uso de un recurso del sistema de transporte, que sin grandes inversiones e infraestructura se reduce la congestión del tráfico vehicular siendo el conocimiento de la ingeniería de tránsito el principal requisito.

La autopista principal en estudio, ubicada en la Panamericana Norte, entre la avenida Tomas Valle y el puente peatonal Control Zarumilla en el distrito de San Martin de Porras, es una de las zonas en donde el mayor tráfico vehicular existe en los días de semana, debido a esto, es de suma importancia proponer una solución para mitigar la congestión mediante un análisis de los niveles de servicio en ambas vías, este análisis pretende dar como propuesta de solución la reversibilidad del sentido de tránsito en las horas pico.

\section{Imagen 1: Carril Reversible en Cerro Centinela}

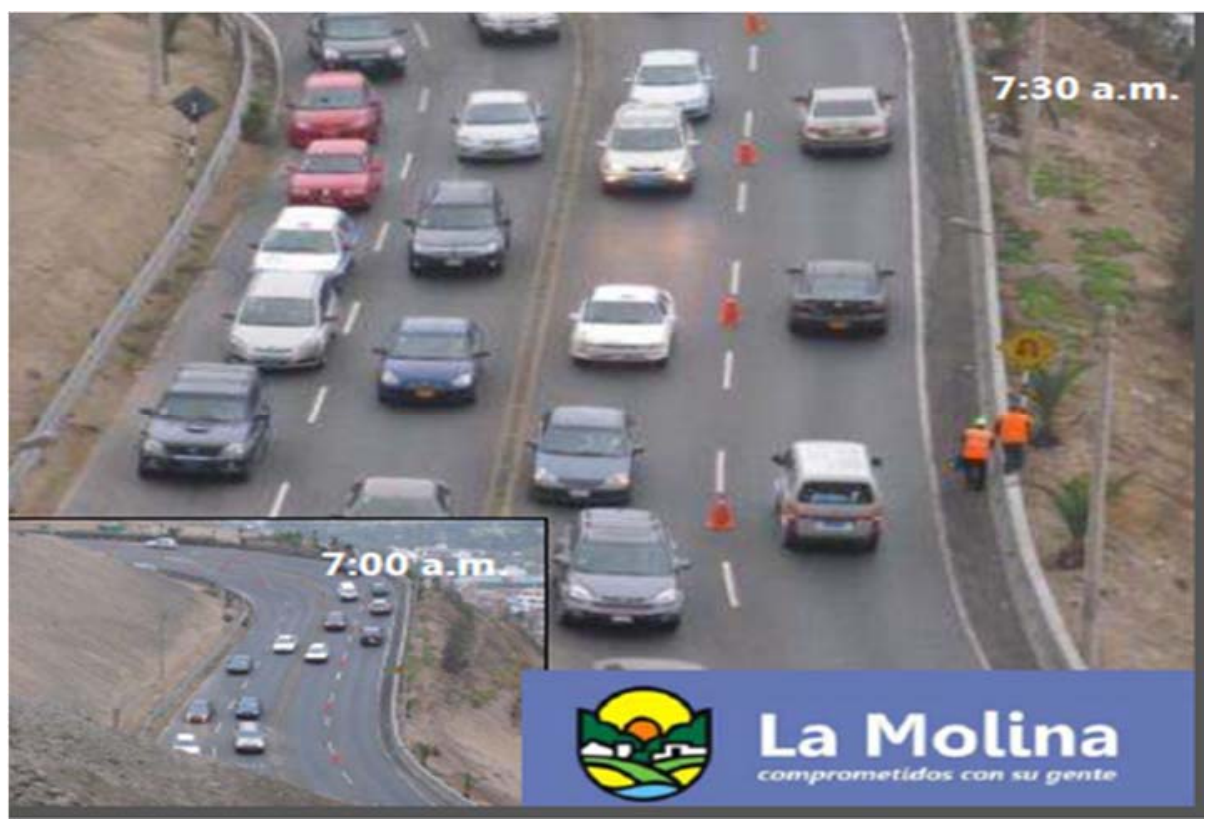

Fuente: Municipalidad de la Molina 
Imagen 2: Plan Verano "Cambio de Sentido"

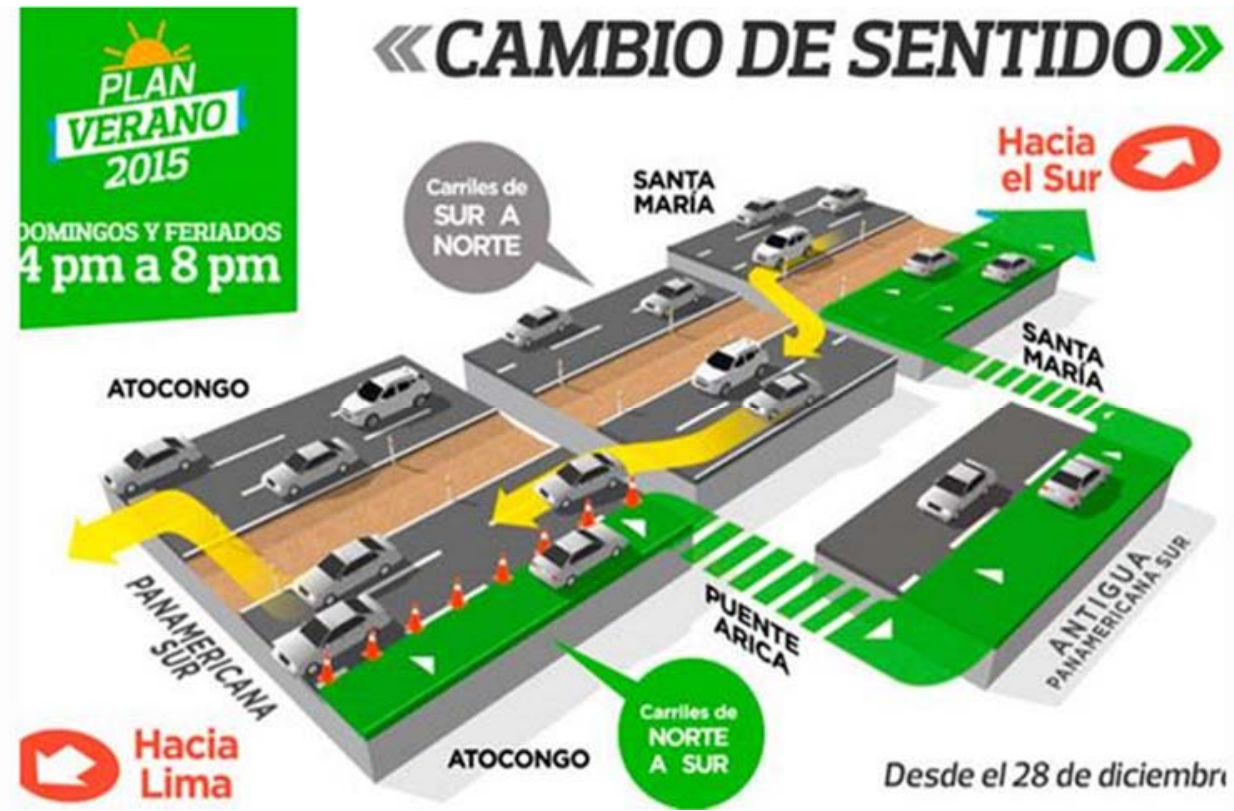

Fuente: Municipalidad Metropolitana de Lima

Imagen 3: Diseño de la zona de trabajo en la primera fase de construcción

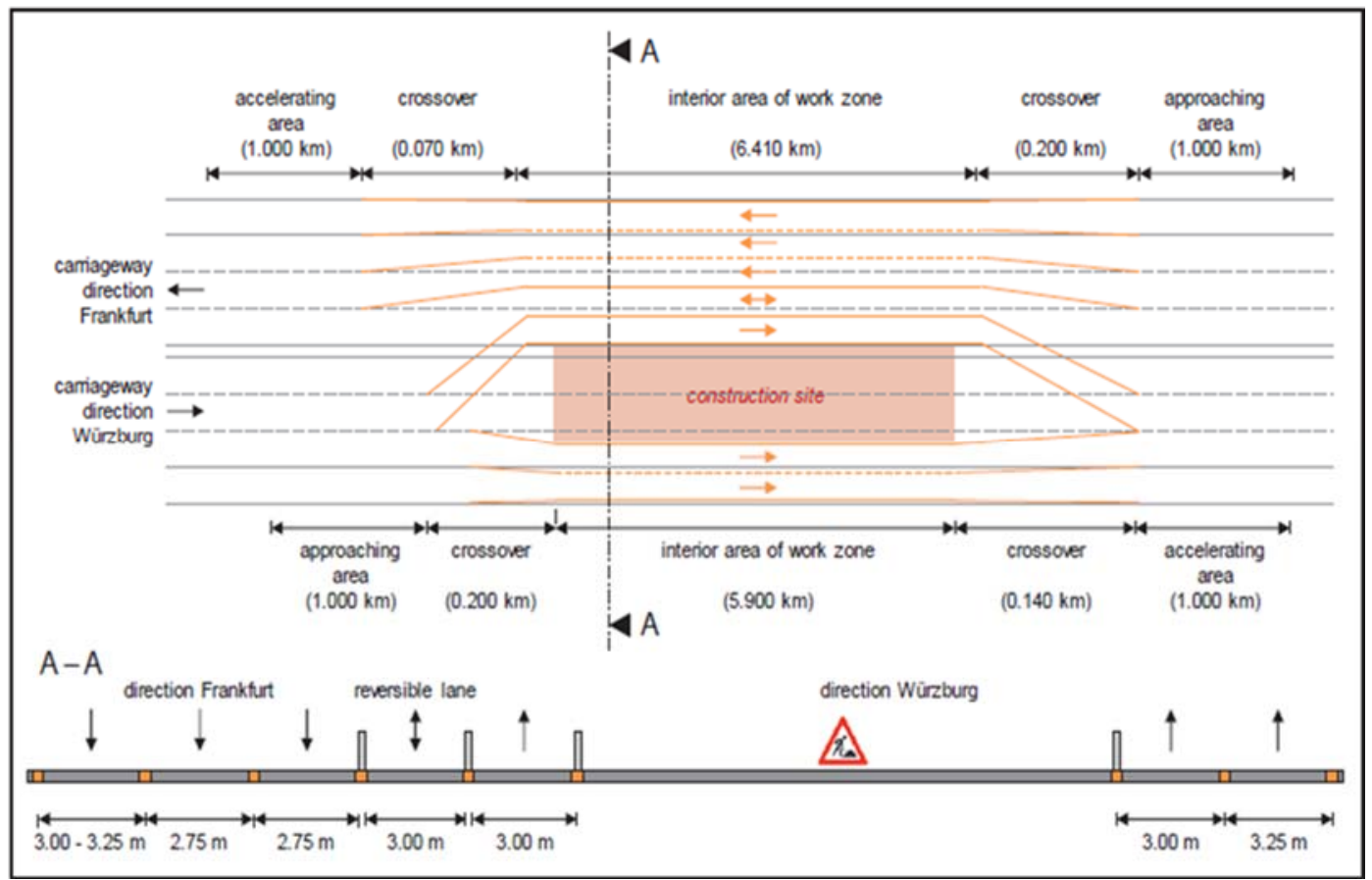

Fuente: Traffic Flow at a Freeway Work Zone with Reversible Median Lane 


\subsection{FUNDAMENTACIÓN}

\subsubsection{Fundamentación del problema}

En los últimos años la congestión vehicular en la ciudad de Lima se ha incrementado desproporcionadamente, constituyendo un peligro para los usuarios, dando como consecuencia el consumo de combustible, polución atmosférica y una progresiva reducción de velocidades, que significa la incrementación del tiempo de viaje a un elevado costo. Según una encuesta elaborada el año 2015 por Ipsos Perú ${ }^{1}$, las personas de la capital pasan en promedio 1 hora 45 minutos diario en la congestión del tránsito, esto quiere decir que en un año perdieron un mes de sus vidas en medio del insoportable tráfico capitalino.

Estos datos son preocupantes, pues los efectos perjudiciales de la congestión recaen directamente sobre los conductores que a la vez lo sufren los pasajeros de transporte masivo. Son muchas las deficiencias que presenta la vialidad urbana hoy en día en la ciudad de Lima, pero la situación se agrava más debido a problemas de diseño, defectuosa información sobre las condiciones de tránsito y la gestión inapropiada de las autoridades competentes. Un caso particular tenemos en Lima Norte, en donde al menos 40 minutos se tarda en cruzar en hora pico los 3.4 kilómetros de Panamericana Norte que hay entre los intercambios viales Tomas Valle y Eduardo de Habich, cuatro veces más de lo que en el año 2008, la Municipalidad de Lima prometió que demoraría hacer todo el trayecto entre Cercado de Lima y Lima Norte.

"Al abrirse esta plataforma elevada (en Habich) el recorrido entre el centro de la ciudad y el cono norte se reduce de 40 minutos a menos de 10 minutos", dijo en ese entonces Carola Cuadros, jefa de Proyectos de la segunda gestión de Luis Castañeda (El Comercio, 01/05/2008).

El ingeniero de tránsito David Fairlie explica que el problema es el diseño de los intercambios viales Eduardo de Habich y Tomas Valle que costó 67 millones y 120 millones de soles respectivamente. Debido a que se trata de una autopista, el alto flujo de vehículos sí ameritaba una obra de esta magnitud, pero cuando se construyeron estos intercambios fusionaron seis carriles a solo tres, generando un cuello de botella que perjudica el tránsito desde varios kilómetros antes. (Ver Imagen 4)

\footnotetext{
1 Web RPP, fuente IPSOS,.
} 
"Eso no tiene sentido. El manual de diseño del Ministerio de Transportes establece que cuando se juntan dos vías tienen que continuar con un número de carriles igual a la sumatoria o, en el peor de los casos, la suma menos uno. Se necesitaría como mínimo cinco carriles”, explicó David Fraile.

Por ello a partir de la investigación de esta situación se propone reorganizar el sistema de vías para el eficiente flujo de transporte público y privado mediante la reversibilidad del sentido de tránsito en horas pico de la Panamericana Norte, entre la avenida Tomas Valle y Puente Control Zarumilla en el distrito de San Martin de Porras.Según la unidad de transporte CEPAL en su libro Congestión del Transito menciona "Esta medida puede traer un importante alivio a la congestión y son en general de bajo costo, siendo el conocimiento de la ingeniería de transito el principal requisito".

Imagen 4: Cuello de Botella de 6 a 3 carriles, cerca de la municipalidad SMP

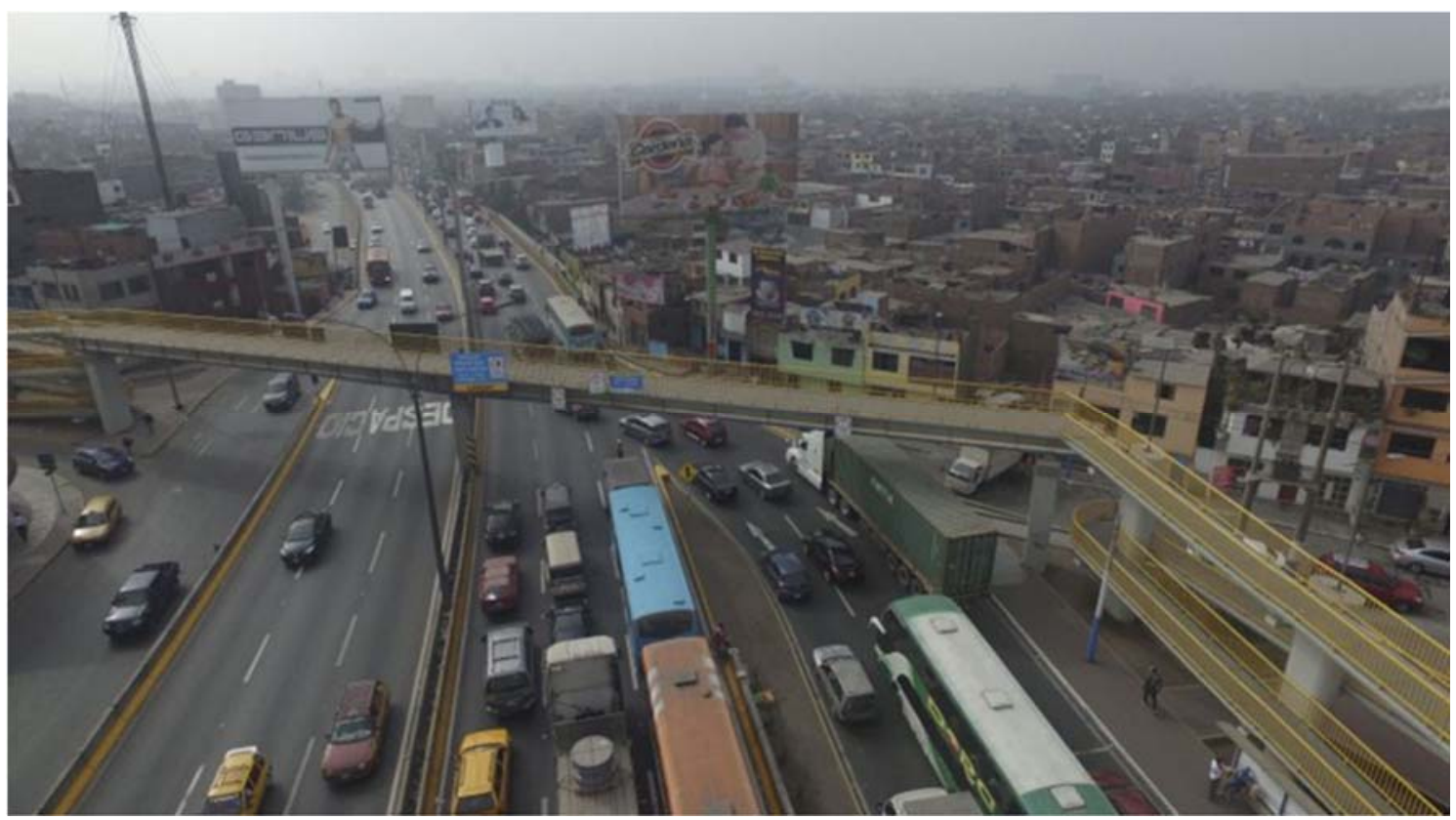

Fuente: El Comercio

\subsubsection{Hipótesis}

La reversibilidad de carril de la autopista de Norte - Sur en la Panamericana Norte entre los tramos del intercambio vial Tomas Valle y el puente peatonal control Zarumilla, mejorará los niveles de servicio de un $\mathrm{F}$ a $\mathrm{D}$ en horas pico de la mañana. Además de balancear los niveles de servicio en ambos sentidos ya que la autopista de Sur - Norte los niveles de servicio están entre un B y C. (Ver Imagen 5). 
Debido a esta explicación tentativa se debe verificar las vías mencionadas balanceando los niveles de servicios con la propuesta de reversibilidad de una de las vías con menor grado de saturación en la hora pico de las mañanas.

Imagen 5: Ilustración de los carriles de ida y regreso de Norte y Sur.

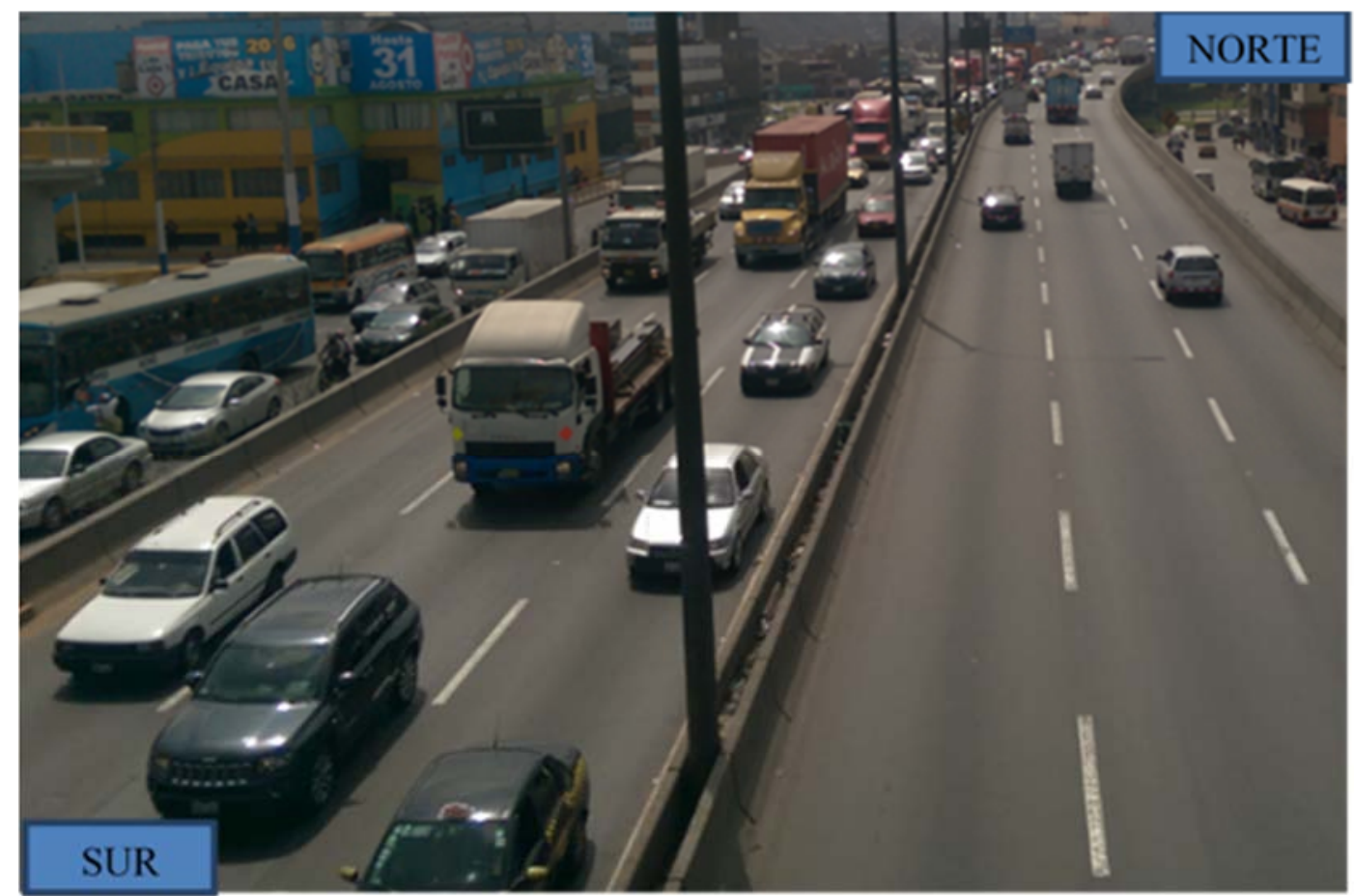

Fuente: Propia

\subsection{OBJETIVOS}

\subsubsection{Objetivo General:}

El objetivo del presente estudio propone mejorar los niveles de servicio en la Panamericana Norte, entre la avenida Tomas Valle y el puente Control Zarumilla en el distrito de San Martin de Porras mediante la reversibilidad de sentido del tránsito mitigando el congestionamiento vehicular en hora pico de la mañana, utilizando el manual de capacidad de carreteras HCM 2010. 


\subsubsection{Objetivos Específicos e Indicadores de logro}

\begin{tabular}{|c|c|}
\hline Objetivos específicos & Indicadores de Logro \\
\hline $\begin{array}{l}\text { a. Recopilar información y complementarla } \\
\text { conjuntamente con el manual de dispositivos } \\
\text { viales de Perú y otras normativas o manuales } \\
\text { extranjeros. }\end{array}$ & $\begin{array}{l}\text { Desarrollar una base de datos con la } \\
\text { información recopilada a través de las } \\
\text { entrevistas a las entidades encargadas } \\
\text { del diseño, construcción y operación } \\
\text { de la vía en estudio. }\end{array}$ \\
\hline $\begin{array}{l}\text { b. Recolectar datos de campo en la Panamericana } \\
\text { Norte, entre la avenida Tomas Valle y puente } \\
\text { Control Zarumilla para evaluar y proponer el } \\
\text { carril reversible en hora pico. }\end{array}$ & $\begin{array}{l}\text { Realizar un trabajo de campo } \\
\text { mediante aforos vehiculares y } \\
\text { geometría de la vía en estudio usando } \\
\text { formato de aforos. }\end{array}$ \\
\hline $\begin{array}{l}\text { c. Calcular el nivel de servicio actual de las vías de } \\
\text { los dos sentidos (Norte-Sur, Sur - Norte) } \\
\text { mediante el manual del HCM } 2010 \text { para } \\
\text { describir una categoría operativa que tiene la vía } \\
\text { en estudio. }\end{array}$ & $\begin{array}{l}\text { Clasificar el nivel de servicio de las } \\
\text { vías de estudio, tanto para Sur-Norte } \\
\text { y Norte-Sur }\end{array}$ \\
\hline $\begin{array}{l}\text { d. Identificar puntos críticos en las intersecciones } \\
\text { de mayor congestión de la arteria principal } \\
\text { (Tramo Tomas Valle }- \text { puente Control } \\
\text { Zarumilla, Panamericana Norte) }\end{array}$ & $\begin{array}{l}\text { Evaluar rutas de entradas y salidas } \\
\text { vehiculares hacia la vía de estudio en } \\
\text { ambos sentidos. }\end{array}$ \\
\hline $\begin{array}{l}\text { e. Aplicar los niveles de servicio con la gestión } \\
\text { actual y de reversibilidad de carriles en hora pico } \\
\text { aplicando el manual de capacidad de carreteras } \\
\text { HCM } 2010\end{array}$ & $\begin{array}{l}\text { Generar una validación de } \\
\text { propuesta mediante el manual } \mathrm{HCM} \\
2010 \text { en gabinete. }\end{array}$ \\
\hline $\begin{array}{l}\text { f. Modelar el caso de estudio a través del Software } \\
\text { de simulación Aimsun 8, aplicando la gestión de } \\
\text { reversibilidad a nivel mesoscópico }\end{array}$ & $\begin{array}{l}\text { Evaluar los resultados más } \\
\text { importantes del software, tanto como } \\
\text { densidad, velocidad y comparar los } \\
\text { resultados con la metodología de } \\
\text { flujos ininterrumpidos del HCM } \\
2010 \text {. }\end{array}$ \\
\hline
\end{tabular}




\subsection{METODOLOGÍA}

Para lograr todos los objetivos de la tesis, este trabajo pasará por distintas etapas descritas a continuación.

Como primer paso, se procederá con la inspección visual del estado actual al entorno de la vía congestionada y la vía libre que esta paralela a esta, con la finalidad de satisfacer los requisitos necesarios para el caso de estudio, tales como:

- Características geométricas de la vía.

- Flujos de entrada y salida vehicular.

- Evaluación de la vía en hora pico.

- Validación de datos del reporte de tráfico.

El segundo paso es obtener los datos necesarios a recolectar en el campo, como la longitud de colas, los aforos vehiculares, tiempo de viajes, entre otros. Para la recolección de datos se emplean herramientas de trabajos como las cámaras filmadoras, cintas métricas, cronómetros y otros elementos necesarios.

Finalizado el registro, se continuará con el procesamiento de datos para construir el modelo mesoscópico a través del HCM 2010 (Highway Capacity Manual) y se verificará los niveles de servicio que tienen ambos sentidos tanto en la gestión actual como en la de carril reversible en hora pico. Además, se comparará los resultados con el software Aimsun, una herramienta de modelación mesoscópico que permite simular operaciones de red que pueden influir de manera más realista posible en cualquier tipo de gestión de tráfico.

\subsection{ALCANCE}

El alcance del área de estudio tendrá lugar en la vía de la Panamericana Norte, entre el intercambio vial Tomas Valle y puente Control Zarumilla en el distrito de San Martin de Porras como se puede mostrar en la imagen 6. 


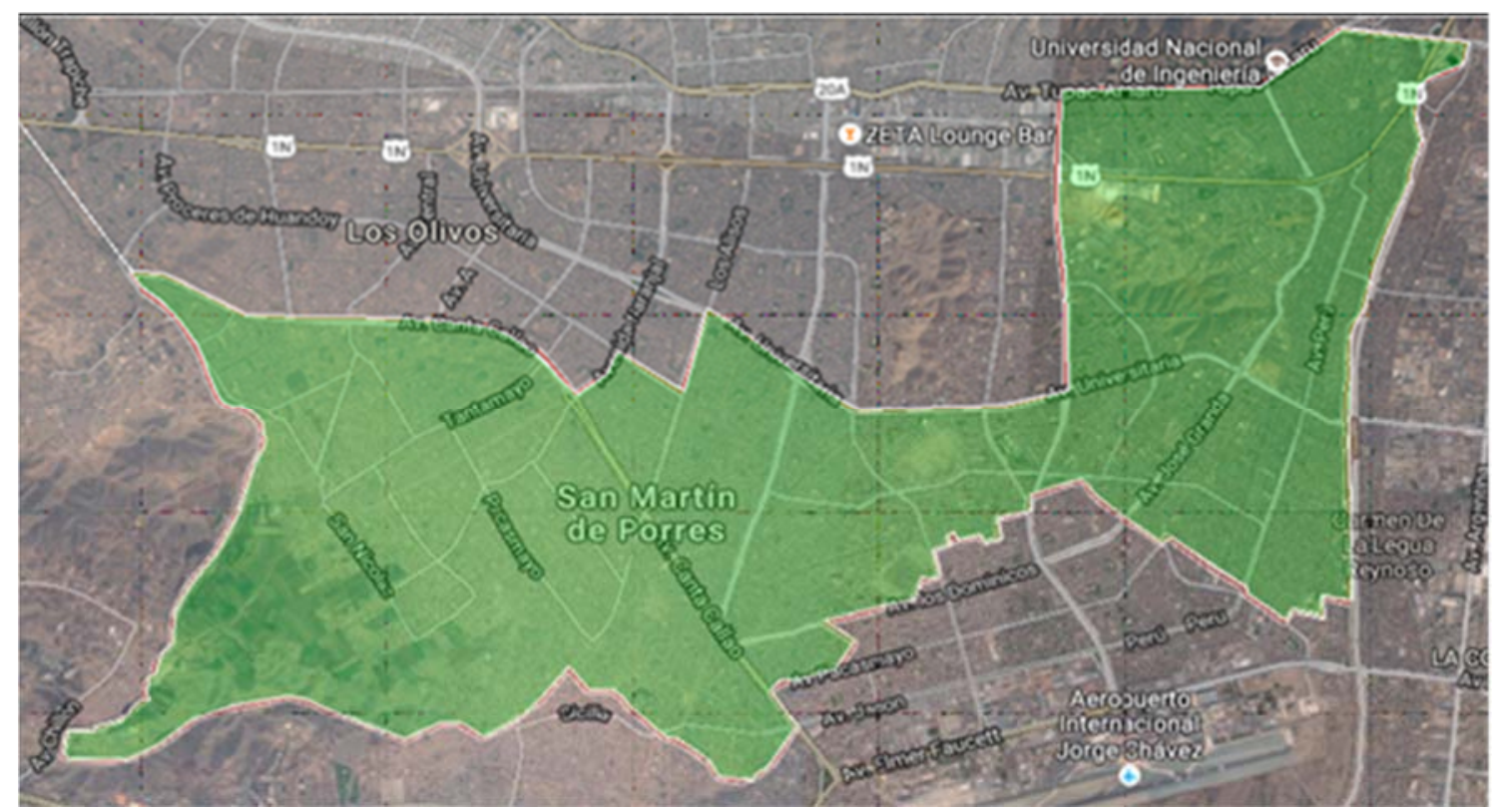

Fuente: Google maps

Según la Policía de Tránsito existen 134 puntos críticos de congestión vehicular identificados en 33 distritos de Lima Metropolitana. La mayoría de estos se concentran en cinco distritos: Cercado, San Martín de Porres, San Isidro, Rímac y La Victoria.

Manejar o viajar por la Panamericana Norte en hora 'pico' es como entrar a la boca del lobo. Los autos privados, camiones, buses, cústeres y combis compiten por avanzar al menos una cuadra. La congestión más densa ocurre entre las 7 y 10 de la mañana, menciona Jesica León, redactora del diario La República. (Fuente: http://larepublica.pe/07-03-2015/congestion-vehicular-se-concentra-en-134-puntoscriticos-de-lima)

El mayor congestionamiento vehicular en el distrito de San Martín de Porras, se da en el tramo de la Panamericana Norte desde el puente control Zarumilla y la Av. Tomas valle, como se puede apreciar en la imagen 7. 


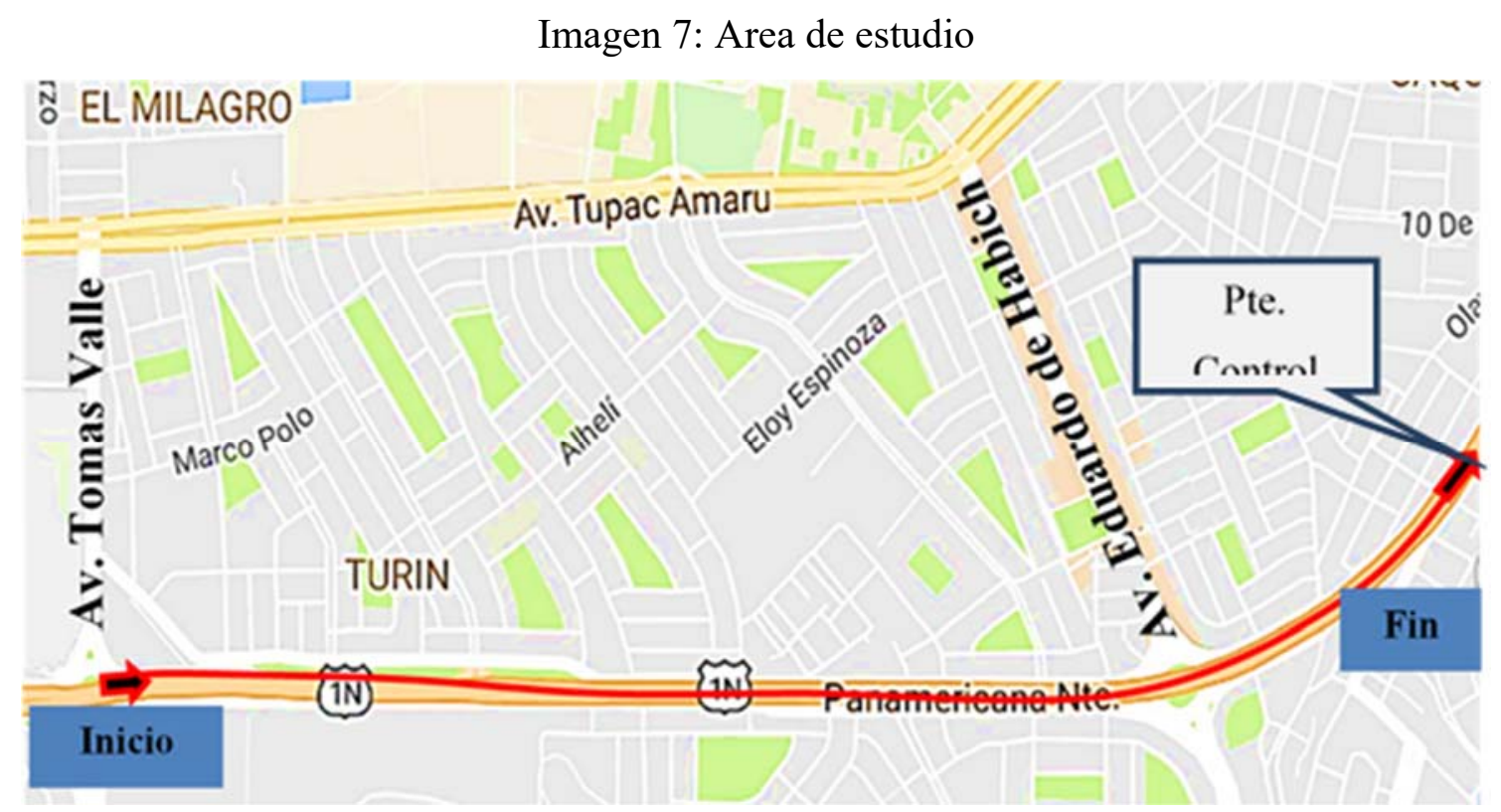

Fuente: Google maps 


\section{CAPITULO II: MARCO TEÓRICO}

\subsection{INGENIERÍA DE TRÁNSITO}

\subsubsection{Congestión vehicular}

La palabra "congestión" es utilizada frecuentemente en el contexto del tránsito vehicular, tanto por técnicos como por los ciudadanos en general. El Diccionario de la Lengua Española ${ }^{2}$ la define "congestionar" a "obstruir o entorpecer el paso, la circulación o el movimiento de algo" que, en nuestro caso, es el tránsito vehicular.

Habitualmente se entiende como la condición en que existen muchos vehículos circulando y cada uno de ellos avanza lenta e irregularmente. Estas definiciones son de carácter subjetivo y no conllevan una precisión suficiente.

La congestión se presenta por diversos motivos, las causas más relevantes son las siguientes:

Características del transporte urbano que provocan la congestión: La demanda de transporte es "derivada", es decir, pocas veces los viajes se producen por un deseo intrínseco de desplazarse; generalmente, obedecen a la necesidad de acceder a los sitios en que se llevan a cabo las distintas actividades: trabajo, compras, estudio, recreación, descanso, y otros, todas las cuales se realizan en lugares diferentes.

El problema es creado principalmente por los automóviles: La existencia de un número excesivo de vehículos, especialmente los de transporte público contribuyen a agravar la congestión. En el área del transporte urbano de pasajeros, una desregulación amplia normalmente se traduce en una acentuada expansión de las flotas de buses y taxis y un deterioro del orden y la disciplina asociadas con su operación.

La condición de las vías y las prácticas de conducción contribuyen a la congestión: La conducción imprudente de algunos conductores y la mala o inadecuada orientación de las señales de tránsito, producen accidentes que conllevan al congestionamiento de la vía.

2 (Real Academia Española - RAE, 2001) 


\subsubsection{Tipos de Modelación del tráfico}

Los modelos empleados para los estudios de los proyectos se pueden clasificar de diferentes formas dependiendo del nivel de análisis y detalles que se quiera lograr. En la imagen 8 , al lado izquierdo se presenta la clasificación debido a su naturaleza, tales como el modelo continuo y discreto. En la sección central se divide según el nivel de detalle. Y al lado derecho; según la dependencia al tiempo y probabilidad (modelo estocástico y determinístico).

Imagen 8: Tipos de modelos considerados

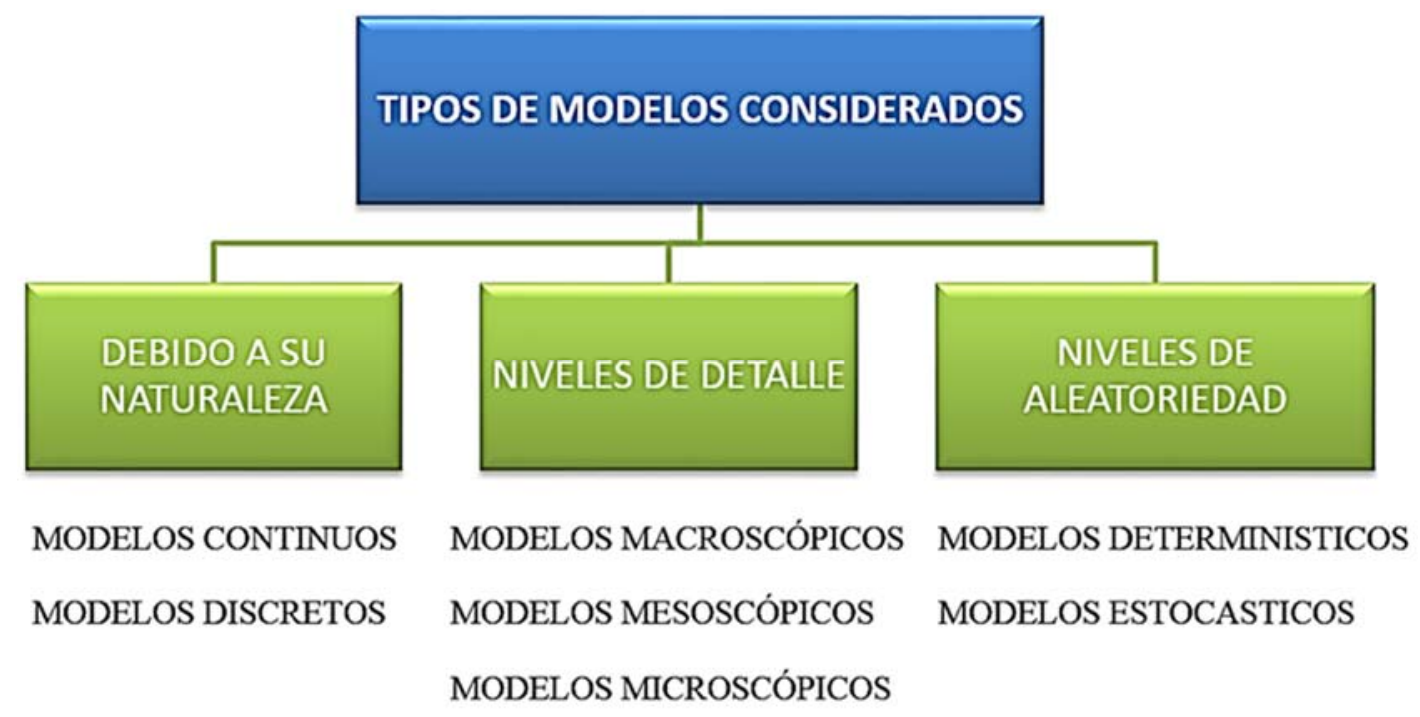

Fuente: Elaboración Propia

\section{a-1 Modelos Discretos}

Estos modelos se caracterizan por considerar la simulación del proyecto, una variación instantánea de los parámetros de estudio para cada intervalo de tiempo (Carvalho, 2015). Por ejemplo, el número de vehículos en una determinada hora del día. Asimismo, este modelo toma en cuenta el comportamiento de los conductores que sufren cambios en intervalos de tiempo. Ellos deciden qué acción tomar frente a cada situación en que se encuentren (Arrieta, 2013). 


\section{a-2 Modelos Continuos}

Estos se basan en la variación continua de los parámetros a lo largo del tiempo de estudio. Cambian constantemente en relación al tiempo. Por ejemplo, los vehículos durante su circulación dentro del tráfico cambian de velocidad, posición, etc. (Aguirre, 2015).

Luego de haber presentado los dos primeros modelos, se debe indicar que las simulaciones realizadas podrán tener distinta naturaleza (discreta o continua), en función a lo que se desea estudiar y se complementarán de manera simultánea según el objetivo del proyecto. Los modelos, también se pueden clasificar por el tipo de evento o aleatoriedad de sus variables, tales como los modelos determinísticos y estocásticos.

\section{b-1 Modelos Macroscópicos}

La principal característica de estos modelos es la representación del flujo vehicular en forma genérica. Entre las consideraciones a tener están la velocidad espacial al flujo y la densidad del tráfico. Si bien es cierto que en este tipo de modelo el nivel de detalle no es muy profundo, los resultados obtenidos podrían ser beneficiosos cuando se trata de proyectos de suma importancia en cuanto a eficiencia, ya que implica un menor tiempo de análisis.

\section{b-2 Modelos Microscópicos}

Representar la realidad mediante una interacción vehicular, a través de asignaciones individuales a los vehículos y peatones, es una característica de este modelo (Mathew \& Krishna, 2006). Por tal motivo, se pueden obtener resultados con mayor exactitud mediante un correcto estudio de los datos de campo y una adecuada estimación del tiempo invertido. Por lo general se requiere de un mayor tiempo que el empleado en un modelo macroscópico (Suárez, 2007).

\section{b-3 Modelos Mesoscópicos}

Este modelo abarca algunas características de los modelos macroscópicos y microscópicos. Sigue el patrón de considerar pelotones de vehículos como una unidad, mientras que los movimientos de giro, tiempo de entrada y salida son determinados de manera singular según el mecanismo simulado (Arrieta, 2013). En la imagen 9 se 
observan las consideraciones pertenecientes a cada modelo, según el nivel de detalle de la simulación.

\section{c-1 Modelos Determinísticos}

Están basados en un estudio analítico, el cual se rige bajo ecuaciones matemáticas que no varían en relación al tiempo; es decir, no dependen de la incertidumbre. Por ello, se obtienen los mismos resultados para los mismos datos de entrada dentro del análisis del proyecto (Starfield, 2005). SYNCHRO es el software más común que sigue el análisis determinístico.

\section{c-2Modelos Estocásticos}

A diferencia del modelo determinístico, el estocástico si presenta variabilidad den las respuestas obtenidas de acuerdo a los datos de entrada y al tiempo de análisis del proyecto. Vissim es uno de los softwares que cumple con estas condiciones (Howard \& Karlin, 1998). Por ello, las simulaciones desarrolladas por los softwares que se basan en configuraciones matemáticas se asocian a los modelos estocásticos. Estos permiten obtener resultados más cercanos a la realidad con un cierto nivel de aleatoriedad según los datos de ingreso, mientras que los modelos determinísticos brindan resultados en base a ecuaciones matemáticas que a veces no se asemejan a la realidad pues desarrollan una única respuesta.

Imagen 6: Principales estrategias de simulación del tráfico

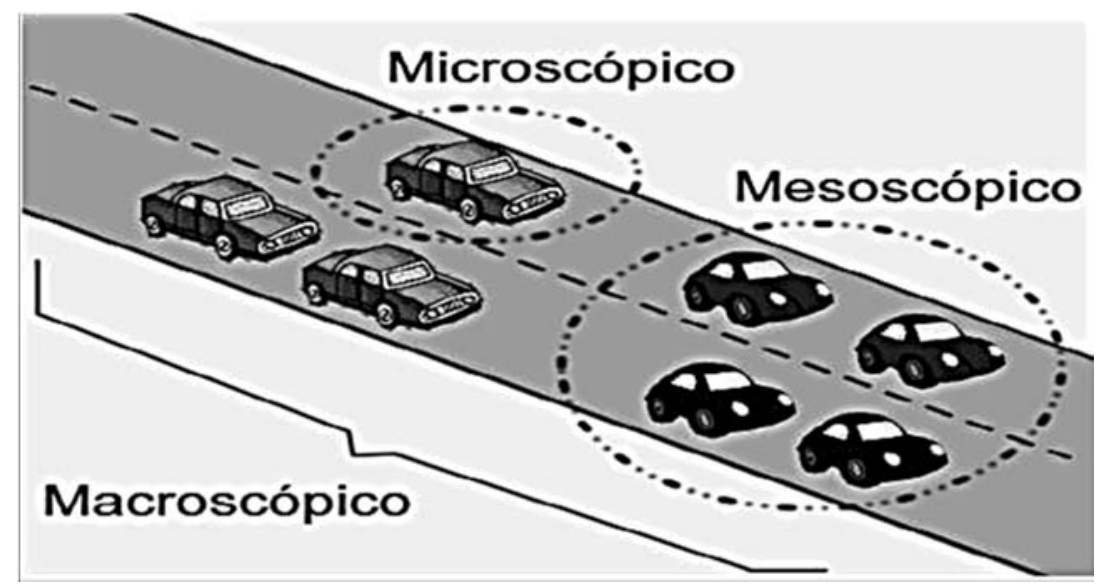

Fuente: Micro simulación del tráfico en intercesiones PUCP 


\subsubsection{Paraderos de servicio público}

Es el espacio de la vía pública técnicamente calificado, autorizado y adecuado por la municipalidad distrital, con el objeto de que la persona jurídica preste el servicio especial, estacionándose temporalmente a la espera de pasajeros. Los paraderos autorizados del servicio especial serán considerados como zonas rígidas para todos aquellos vehículos que no se encuentren autorizados para su uso.

\subsection{CONCEPTO DEL MANUAL HCM 2010}

\subsubsection{Guía de Uso del Manual HCM 2010}

El Manual de Capacidad de Carreteras 2010 es la quinta edición de este documento de referencia fundamental que viene desarrollándose desde el año 1950. Al igual que sus predecesores, el HCM 2010 ha sido revisado significativamente para incorporar las últimas investigaciones sobre la capacidad de las carreteras y la calidad de sus servicios. Esta nueva edición se reorganizó con la finalidad de hacerlo más comprensible y práctico de acuerdo a las necesidades de los usuarios y a los nuevos proyectos presentes hoy en día.

El HCM 2010 ha añadido elementos nuevos a partir de proyectos de investigación finalizados desde la publicación del HCM 2010 además está destinada a favorecer a los analistas a considerar toda una amplia gama de medidas de rendimiento y optimización al momento de evaluar las capacidades de las carreteras. Los principales cambios que se han hecho con respecto al HCM 2010 están en las diferentes metodologías para medir los rendimientos de las carreteras.

La guía es el punto de partida para aprender a utilizar de manera adecuada esta edición del HCM 2010, pues su propósito es proporcionar un conjunto de metodologías y asociarlos a los procedimientos de solicitud que se quiere, todo esto con la finalidad de evaluar el desempeño de la autopista en estudio y mejorar la calidad de las vías bajo el concepto de niveles de servicio. Esta calidad se describe que tan bien opera un servicio de transporte desde la perspectiva del viajero o usuario.

Los objetivos que tiene este Manual del HCM 2010 son: 
Definir las medidas de rendimientos y describir los métodos claves para las características del tráfico de la vía en estudio.

Proporcionar metodologías para estimar y predecir las medidas de rendimiento.

Explicar las metodologías a un nivel de detalle que permita a los lectores comprender los factores que afectan la operación multimodal.

A pesar de que el HCM 2010 no establece una norma legal para el diseño y construcción de carreteras, esta presenta las mejores técnicas disponibles en el momento de la publicación para determinar la capacidad y los niveles de servicio (LOS).

\subsubsection{Aplicaciones}

El manual de capacidad de carreteras (HCM 2010) se puede aplicar al área de transporte que van desde el más alto nivel de detalle hasta la muy generalizada, o a elementos del sistema de caminos que van desde puntos individuales hasta a un total sistema de transporte.

Para este caso de estudio el HCM 2010 se verá aplicado en el nivel operativo que tiene ya la autopista construida, bajo el concepto de los niveles de servicio, que va ser el resultado de un conjunto dado de insumos que determinaran las características necesarias para lograr un buen flujo vehicular en el caso de estudio.

Los modos de desplazamiento cubiertos por el HCM 2010 en el caso de estudio son el automóvil incluyendo otros vehículos como los camiones, vehículos de recreación, autobuses, buses interurbanos, camiones de 2 o más ejes, así como los vehículos de transporte público. Cualquier aplicación del HCM 2010 puede ser analizado en diferentes niveles de detalles, dependiendo la finalidad que tiene el caso de estudio y la cantidad de información que se dispone.

El HCM 2010 define en tres niveles principales de análisis:

- Análisis Operativo.

- Análisis de Diseño.

- Planificación y análisis de ingeniería preliminar. 
En el presente caso de estudio nos centraremos en el análisis operativo, que por lo general se centra en las condiciones actuales o de corto plazo, además de la participación de entradas detalladas de los procedimientos del HCM 2010, con o sin un uso mínimo de valores predeterminados.

El objetivo del análisis operativo es aportar información para las decisiones sobre si hay una necesidad de mejoras en un punto existente que se requiera implementar medidas de solución. Ocasionalmente, se realiza un análisis para determinar si se necesita un estudio más amplio en la planificación. En el caso de que falle o no se cumpla con un nivel de servicio deseado, el HCM 2010 analiza alternativas que ayudan a los analistas a tener un flujo adecuado para la vía en estudio, estas alternativas implican a menudo un análisis de un carril reversible, dispositivos de tráfico alternativo, temporización de las señales de tránsito o su eliminación gradual, espaciamiento y ubicación de las paradas de autobús y la frecuencia de autobuses.

\subsubsection{Volumen de transito}

El volumen de tránsito es definido como el número de vehículos que pasan en un determinado punto durante un intervalo de tiempo. La unidad para el volumen es simplemente "vehículos" o "vehículos por unidad de tiempo". Un intervalo común de tiempo para el volumen es un día, descrito como vehículos por día. Los volúmenes diarios frecuentemente son usados como base para la planificación de las carreteras.

Para los análisis operacionales, se usan los volúmenes horarios, ya que el volumen varía considerablemente durante el curso de las 24 horas del día. La hora del día que tiene el volumen horario más alto es llamada "hora pico". Los volúmenes de hora pico son usados como la base para el diseño de carreteras y para varios tipos de análisis operacionales.

Para períodos menores a una hora, generalmente el volumen se expresa como un equivalente horario de las razones de flujo. Por ejemplo, 1,000 vehículo observado en un período de 15 minutos se puede expresar como:

$$
1,000 \mathrm{veh} / 0.25 \mathrm{hr}=4,000 \mathrm{veh} / \mathrm{hr}(\text { Ecu. } 1)
$$

La razón de flujo (v) es 4,000 veh/hr en un intervalo de 15 minutos, en el cual fueron observados 1,000 vehículos. 
La relación entre el volumen horario y la máxima razón de flujo se define como el factor de hora pico (FHP):

$$
\text { FHP = volumen horario / máxima razón de flujo (Ecu. 2) }
$$

Para períodos de 15 minutos, la ecuación se convierte en:

$$
\mathrm{FHP}=\mathrm{V} /(4 \mathrm{X} \mathrm{V} \text { 15) (Ecu. 3) }
$$

Dónde:

$\mathrm{V}=$ Volumen horario $(\mathrm{veh} / \mathrm{hr})$

$\mathrm{V}_{15}=$ Volumen máximo en 15 minutos de la hora (veh)

Según el HCM 2010, el valor del FHP para áreas rurales es de 0.88 y el valor para áreas urbanas es de 0.92. Regularmente para el análisis de carreteras y autopistas se usa el valor de 0.90 , cuando no se dispone de la información de campo para obtener el valor del FHP.

\subsubsection{Capacidad}

Un objetivo principal del análisis de la capacidad es estimar el número máximo de vehículos que una carretera puede acomodar con razonable seguridad durante un período específico de tiempo. Sin embargo, las carreteras generalmente operan pobremente o cerca de la capacidad; son raras las planificadas que operan en el rango correcto. En consecuencia, el análisis de capacidad también estima el aumento de tránsito que una carretera puede acomodar mientras mantiene su nivel de operación prescrito.

\subsubsection{Definición de Capacidad.}

La capacidad es el máximo número de vehículos que pueden circular en un punto dado durante un período específico de tiempo, bajo condiciones prevalecientes de la carretera y el tránsito. Asumiendo que no hay influencia del tránsito más adelante, dentro del punto en análisis.

Las condiciones prevalecientes de la carretera se refieren a características geométricas como el número y uso de carriles, distancia lateral derecha, configuración de carriles y el alineamiento horizontal y vertical. El flujo máximo del tránsito de una carretera es su capacidad, que ocurre cuando se alcanza la densidad crítica y el tránsito se mueve a la 
velocidad crítica. Esto regularmente ocurre en la hora pico del volumen del tránsito, la hora pico es el período más crítico. La capacidad frecuentemente se mide en vehículos por hora (veh/hr).

\subsubsection{Valores de la capacidad para condiciones ideales}

Para autopistas de dos o más carriles los valores básicos de la capacidad bajo condiciones ideales son descritos como sigue:

Condiciones ideales:

- Flujo ininterrumpido.

- $\quad 3.60$ metros de ancho de carril.

- $\quad 1.80$ metros de distancia mínima lateral (lado derecho)

- Sin vehículos pesados.

- Velocidad de diseño $\geq 80 \mathrm{Km} . / \mathrm{hr}$.

- Carencia de restricciones en la distancia de visibilidad de rebase.

- Sin interferencia de paso de peatones.

- En ambas direcciones el tránsito debe ser igual (50/50).

La capacidad de autopistas de tres carriles, según el HCM 2010 es de 4200 (veh/hr/carril) en ambas direcciones. El tránsito puede operar idealmente solo si el ancho de los carriles y de la distancia mínima lateral derecha tiene los valores descritos arriba, de no ser así la velocidad se reduce y aumenta el porcentaje de tiempo utilizado en seguir un vehículo.

\subsubsection{El ratio v/c y su uso}

Un factor crítico en cualquier análisis de capacidad es la proporción de la capacidad de la carretera siendo utilizada como proyección del tránsito. Este valor es el ratio de proporción de flujo para la capacidad de la carretera.

$$
\mathrm{v} / \mathrm{c}=\text { tasa de flujo / capacidad (Ecu. 4) }
$$

Este ratio es usado como una medida de la suficiencia de capacidad existente o propuesta. En concepto un ratio mayor a 1.00 puede existir cuando un flujo de demanda pronosticado es usado para comparar una capacidad existente o estimada. La tasa de flujo nunca puede 
sea mayor que su capacidad. En la misma situación, el ratio v/c mayor a 1.00 predice que la carretera falló, siendo incapaz de descargar la demanda que llega a la sección en servicio. En otras palabras, un valor del ratio v/c mayor igual a 1.00 implica congestionamiento.

\subsubsection{Conceptos de Niveles de Servicio}

En el documento actualizado HCM 2010 se establecen seis niveles de servicio las cuales se encuentran identificados subjetivamente por las letras desde la A hasta la F, donde al nivel de servicio A logra un flujo vehicular completamente libre, mientras que al nivel $\mathrm{F}$ alcanza el flujo forzado, estas reflejan condiciones de utilización a plena capacidad de la vía. Cabe resaltar que al hablar de congestionamiento en una autopista no es hablar de paralización de todo el movimiento del flujo vehicular.

Para el diseño se debe escoger, entre dichos extremos, el nivel de servicio que mejor se adecua a la realidad del proyecto a desarrollar. Como criterio de análisis, se expresa que el flujo vehicular de servicio para diseño debe ser mayor que el flujo de tránsito durante el período de 15 minutos de mayor demanda durante la hora de diseño.

Definición de niveles de servicio: Un nivel de servicio es una designación que describe un rango operativo sobre un tipo particular de una carretera. Las condiciones generales de operación para los niveles de servicio (de A al F), se describen de la siguiente manera.

\section{Nivel de Servicio A}

En la Imagen 10 se aprecia un flujo libre de vehículos, bajos volúmenes de tránsito y relativamente altas velocidades de operación $(90 \mathrm{~km} / \mathrm{hr}$ o más). La demora de los conductores no es mayor al 35\% del total del tiempo de viaje y la razón de flujo total para ambas direcciones es de $490 \mathrm{veh} / \mathrm{hr}$. 
Imagen 10: Nivel de servicio A

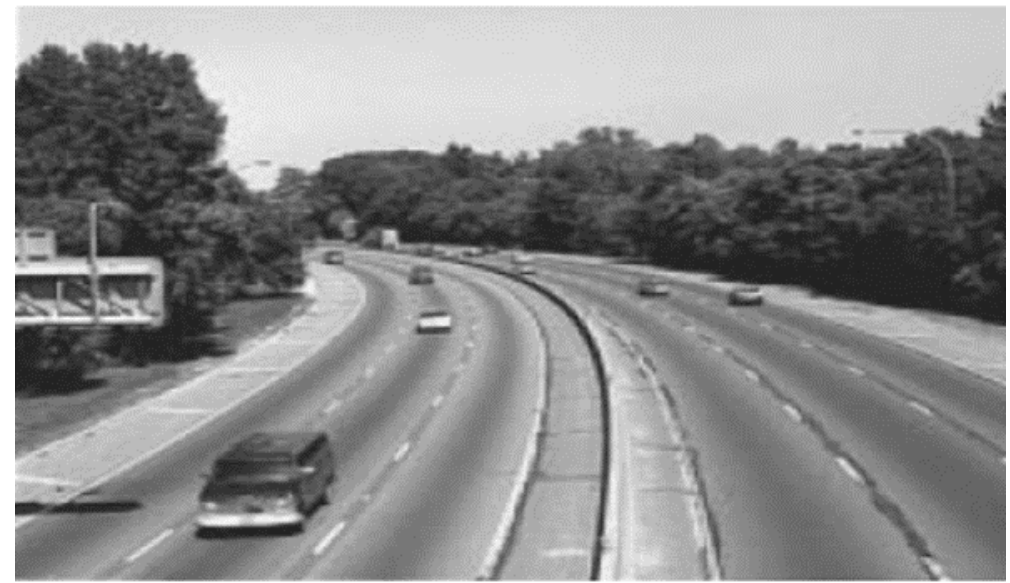

Fuente: HCM 2010

\section{Nivel de Servicio B}

En la imagen 11 se ve un flujo libre razonable, pero la velocidad empieza a ser restringida por las condiciones del tránsito $(80 \mathrm{~km} / \mathrm{hr})$. La demora de los conductores no es mayor al $50 \%$ del total del tiempo de viaje y la razón de flujo total para ambas direcciones es de $780 \mathrm{veh} / \mathrm{hr}$.

\section{Imagen 11: Nivel de servicio B}

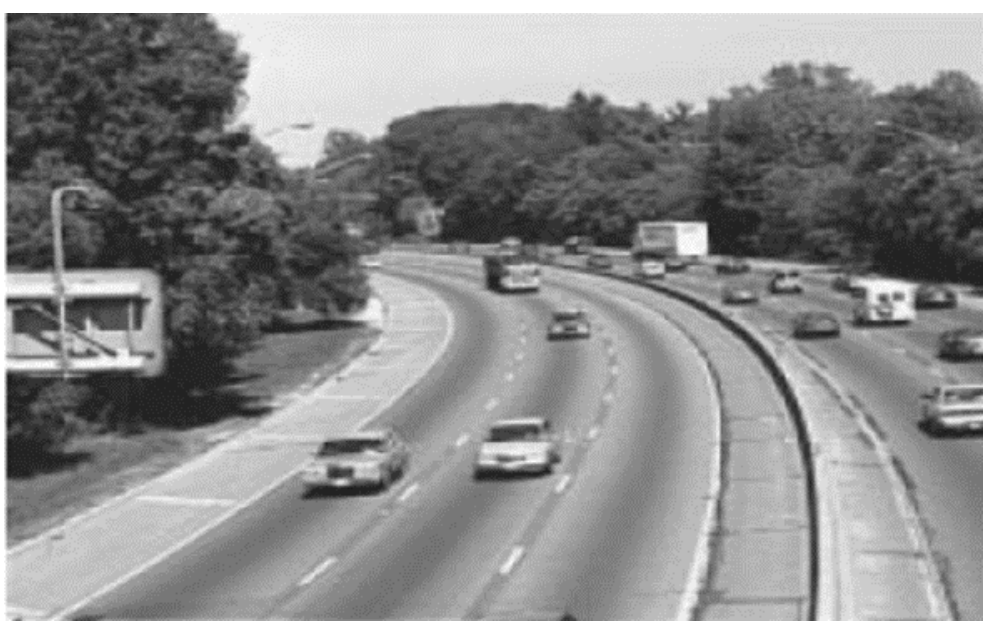

Fuente: HCM 2010

3. Nivel de Servicio C

En la imagen 12 los vehículos se mantienen en zona estable, pero muchos conductores empiezan a sentir restricciones en su libertad para seleccionar su propia velocidad (70 $\mathrm{Km} . / \mathrm{hr}$ ). La demora de los conductores alcanza el 65\% del total del tiempo de viaje y la razón de flujo total para ambas direcciones es de 1,190 veh/hr. 
Imagen 12: Nivel de servicio C

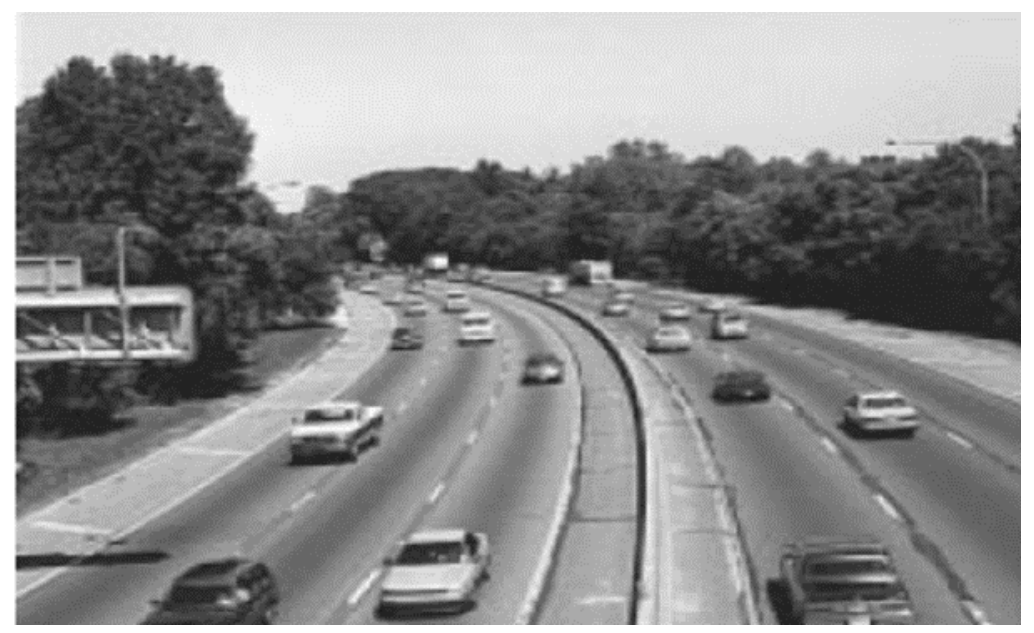

Fuente: HCM 2010

\section{Nivel de Servicio D}

En la Imagen 13 los vehículos se acercan a un flujo inestable, los conductores tienen poca libertad para maniobrar. La velocidad se mantiene alrededor de los $60 \mathrm{~km} / \mathrm{hr}$. La demora de los conductores es cercana al $80 \%$ del total del tiempo de viaje y la razón de flujo total para ambas direcciones es de $1,830 \mathrm{veh} / \mathrm{hr}$.

Imagen 13: Nivel de servicio D

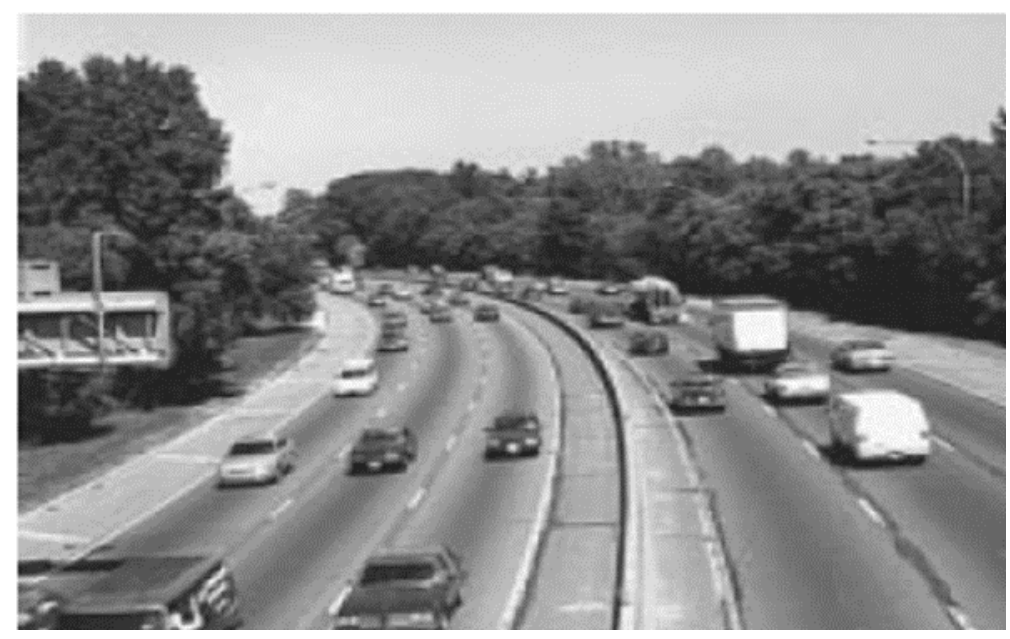

Fuente: HCM 2010

\section{Nivel de Servicio E}

En la imagen 14 los vehículos tienen un flujo inestable, suceden pequeños congestionamientos. La velocidad cae hasta $40 \mathrm{~km} / \mathrm{hr}$. La demora de los conductores es mayor al $80 \%$ del total del tiempo de viaje. 
Imagen 14: Nivel de servicio E

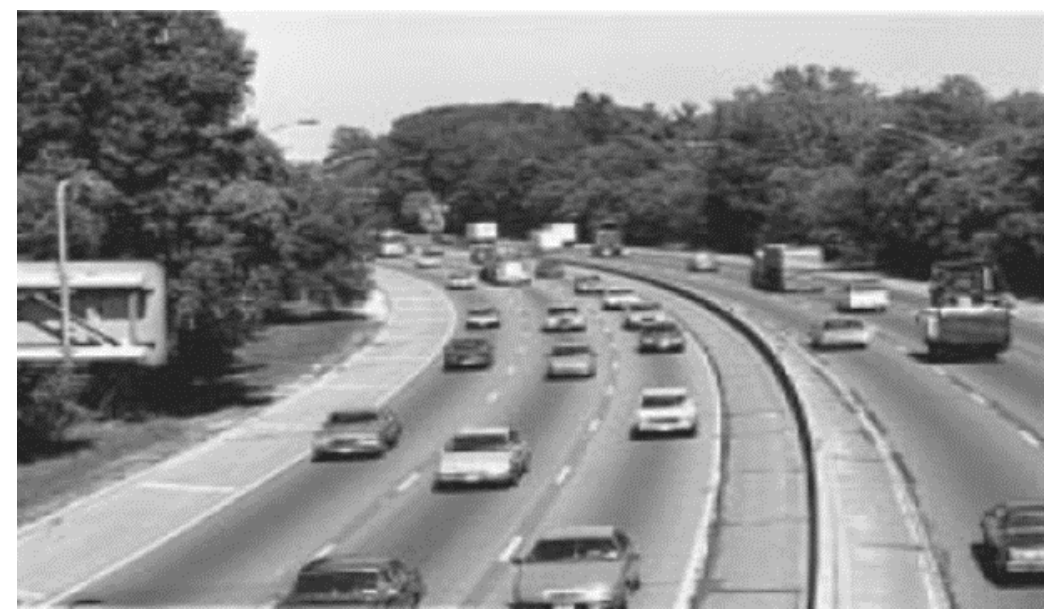

Fuente: HCM 2010

\section{Nivel de Servicio F}

En la imagen 15 se aprecia un Flujo forzado de los vehículos, condiciones de "pare y siga”, congestión de tránsito.

Imagen 15: Nivel de servicio F

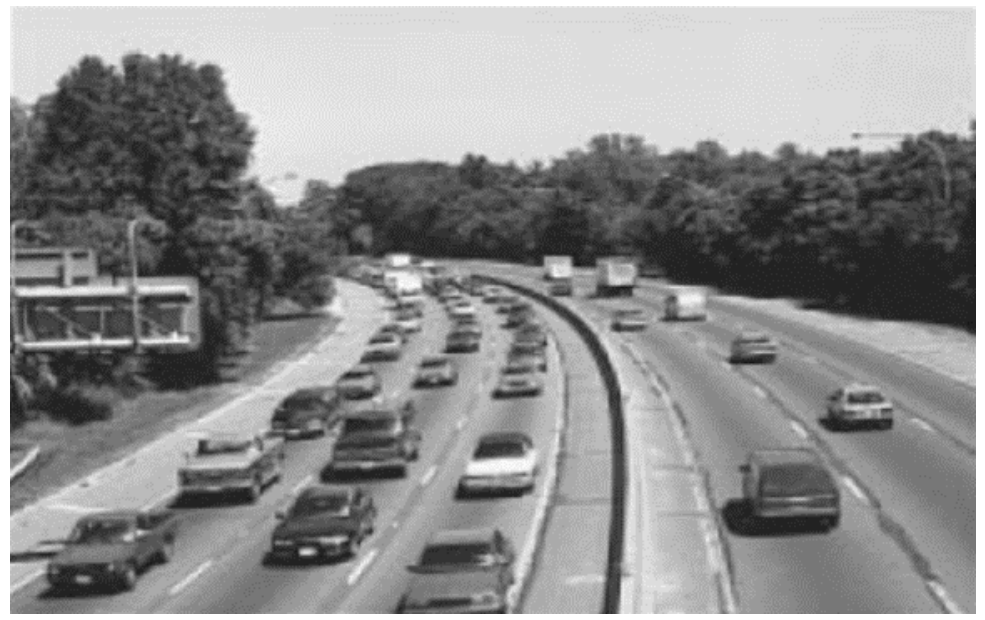

Fuente: HCM 2010

\subsubsection{Concepto de Velocidad}

Aunque los volúmenes de tráfico proporcionan un método para cuantificar los valores de la capacidad, la velocidad es una medida importante de la calidad del servicio de tráfíco proporcionado al usuario. Se define como una velocidad de movimiento expresada como la distancia por unidad de tiempo, generalmente como kilómetros por hora $(\mathrm{km} / \mathrm{h})$. Para caracterizar la velocidad de una corriente de tráfico, debe usarse un valor representativo, 
porque una amplia distribución de velocidades individuales es observable en la corriente de tráfico. Se pueden aplicar varios parámetros de velocidad a un flujo de tráfico. Entre ellos se encuentran los siguientes:

Velocidad de desplazamiento promedio: Una medida de flujo de tráfico basada en el tiempo de viaje observado en una longitud conocida de la carretera. Es la longitud del segmento dividido por el tiempo medio de desplazamiento de los vehículos que atraviesan el segmento, incluyendo todos los tiempos de retardo detenidos. También es igual a la velocidad media espacial.

Velocidad media espacial: Término estadístico que indica una velocidad media basada en el tiempo medio de viaje de los vehículos para recorrer una longitud de carretera. Se denomina velocidad media espacial porque el tiempo promedio de desplazamiento pesa el promedio por el tiempo que cada vehículo gasta en el segmento o espacio de la calzada definida.

Velocidad media temporal: La media aritmética de las velocidades de los vehículos observados pasa un punto en una carretera; También conocida como la velocidad media del punto. Las velocidades individuales de los vehículos que pasan en un punto se registran y promedian aritméticamente.

Velocidad de flujo libre: Es la velocidad media de los vehículos en un segmento determinado medida en condiciones de bajo volumen, cuando los conductores son libres de conducir a la velocidad deseada y no están limitados por la presencia de otros vehículos o dispositivos de control de tráfico en sentido descendente (es decir, señales de tráfico, rotondas o Señales de PARE).

Velocidad media de operación: Una medida de flujo de tráfico basada en la observación de los tiempos de recorrido de los vehículos que atraviesan una sección de carretera de longitud conocida es la longitud del segmento dividido por el tiempo medio de funcionamiento de los vehículos que atraviesan el segmento. El tiempo de funcionamiento incluye solamente el tiempo durante el cual los vehículos están en movimiento.

Para la mayoría de los procedimientos de HCM 2010 que usan la velocidad como medida de servicio, la velocidad media de desplazamiento (o su equivalente, velocidad media 
espacial) es el parámetro que los define. En instalaciones de flujo ininterrumpido que funcionan con flujo no saturado, la velocidad media de desplazamiento es igual a la velocidad media de marcha. La velocidad media espacial es siempre inferior o igual a la velocidad media del tiempo, pero la diferencia disminuye a medida que aumenta el valor absoluto de la velocidad. Basándose en el análisis estadístico de los datos observados, esta relación es útil porque las velocidades medias del tiempo son típicamente más fáciles de medir en el campo que las velocidades medias del espacio.

\subsection{FLUJOS ININTERRUMPIDOS PARA AUTOPISTAS DE SEGMENTOS BÁSICOS.}

\subsubsection{Autopista de Segmento Básico}

Las Autopistas de segmento básico se definen como aquellos que son fuera de la influencia de los tejidos de maniobras o rampas de ingreso o de salida. En general, esto significa que la actividad de cambio de carril no está influenciada significativamente por la presencia de rampas y segmentos de tejido. El cambio de carril se dará principalmente por el deseo de los conductores al optimizar su eficiencia.

Para desarrollar la metodología de este tipo de autopistas, debe haber algunas condiciones básicas, como lograr incluir un buen tiempo de tránsito, buena visibilidad, nada de incidentes o accidentes, nada de deterioros del pavimento que sean lo suficientemente graves para afectar la transitividad vehicular. Si ninguna de estas condiciones no existe en la autopista, la velocidad, los niveles de servicio y la capacidad de la propia autopista, pueden ser peores a las predichas por la metodología planteada.

Aparte de las condiciones básicas mencionadas anteriormente, existes otras que se deben ajustar para la aplicación de la metodología como:

No se permiten vehículos pesados en el tráfico de una autopista de segmento básico.

Se necesita un mínimo de 3.6 metros de ancho de los carriles y espacios libres del lado derecho de 1.8 metros. 


\subsubsection{Capacidad en condiciones Base.}

La capacidad de un segmento básico de autopista bajo condiciones de base varía con la velocidad a flujo libre (FFS). Según la imagen 15 para flujos de $75 \mathrm{mi} / \mathrm{h}-70 \mathrm{mi} / \mathrm{h}$, la capacidad es de 2,400 veh/h/c. Para niveles menores de FFS, la capacidad disminuye ligeramente. Por $65 \mathrm{mi} / \mathrm{h}$ de FFS, la capacidad es de 2,350 veh/h/c; de $60 \mathrm{mi} / \mathrm{h}$ de FFS, $2300 \mathrm{veh} / \mathrm{h} / \mathrm{c}$; y para $55 \mathrm{~km} / \mathrm{h}$ de FFS, la capacidad es $2,250 \mathrm{veh} / \mathrm{h} / \mathrm{c}$.

Estos valores representan las normas nacionales. Se debe recordar que la capacidad varía estocásticamente y que cualquier lugar determinado podría tener un mayor o valor menor. También debe recordarse que la capacidad se refiere a la media velocidad de flujo a través de todos los carriles. Por lo tanto, un segmento de autopista básica de tres carriles con respecto a nuestro caso de estudio con $55 \mathrm{mi} / \mathrm{h}$ FFS tendrá una capacidad de base prevista de $3 \times 2,200=6,600 \mathrm{veh} / \mathrm{h} / \mathrm{c}$. Este flujo no sería distribuido de manera uniforme en todos los carriles. Por lo tanto, uno o dos carriles podrían tener un flujo base estable de más de $2.200 \mathrm{veh} / \mathrm{h} / \mathrm{c}$.

\section{Imagen 16: Grafico Velocidad de Flujo Libre vs Capacidad}

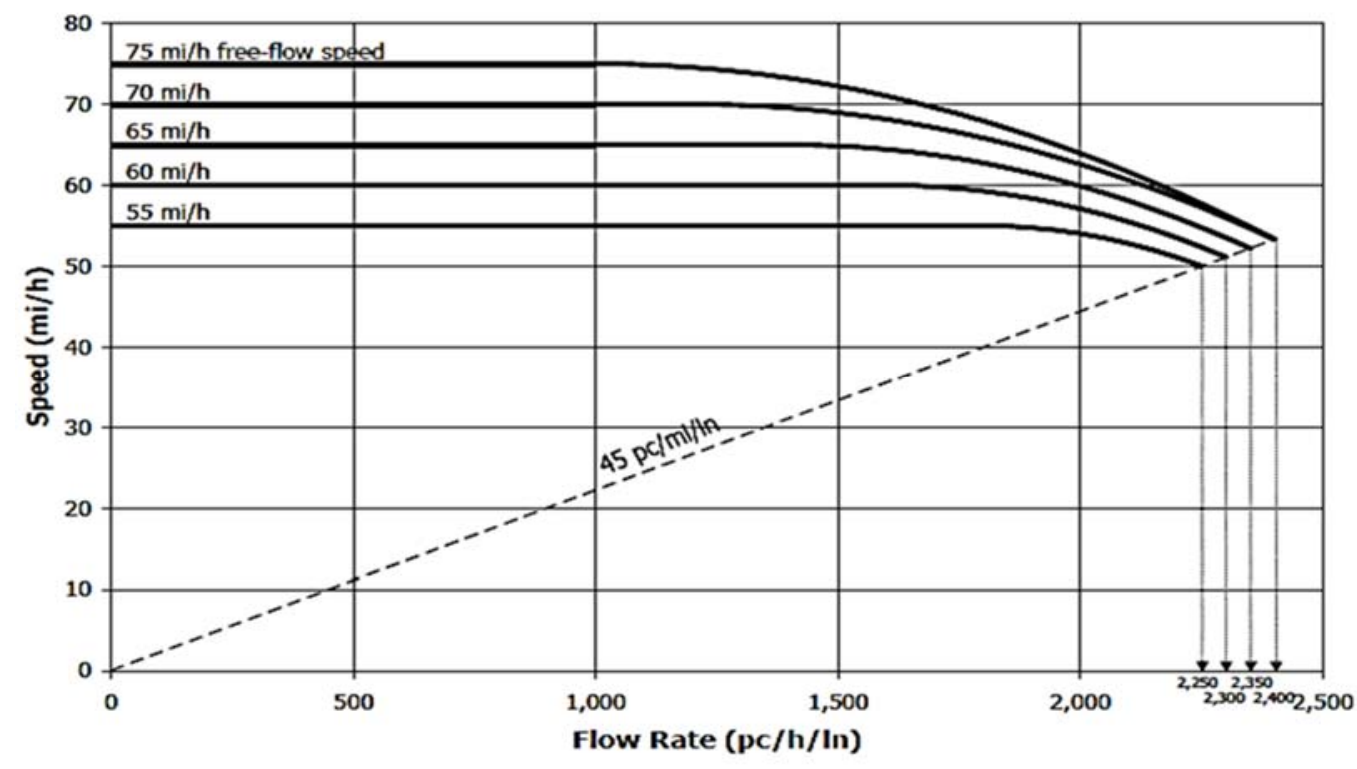

Fuente: HCM 2010

Como se muestra en la Imagen 16, se cree que los segmentos básicos de autopista alcanzan capacidad a una densidad de aproximadamente 45 autos de pasajeros por milla por carril (veh / mi / ln), que puede variar ligeramente de un lugar a otro. A esta densidad, los vehículos están poco espaciados para amortiguar el impacto de cualquier perturbación 
del flujo, tal como un cambio de carril o un vehículo que entra a la autopista, sin causar una interrupción que sea un accidente de tránsito.

\subsubsection{Niveles de servicio para Autopistas de segmentos Básicos}

Los niveles de servicio para autopistas de segmentos básicos se definen por la densidad. Aunque la velocidad es una de las principales preocupaciones de los conductores en relación con la calidad del servicio, sería difícil para los niveles de servicio describirlos mediante el uso de la velocidad, ya que se mantiene constante hasta las tasas de flujo de 1.000 a $1,800 \mathrm{veh} / \mathrm{h} / \mathrm{c}$; La densidad describe la proximidad a otros vehículos y se relaciona con la libertad de maniobra dentro de la corriente de tráfico a diferencia de la velocidad, sin embargo, la densidad es sensible a las tasas de flujo en toda la gama de flujos. En los ejemplos del concepto de niveles de servicio se mostró visualmente los seis Niveles de Servicios (LOS) definidos por la autopista de segmentos básicos. Estos están definidos para representar rangos razonables en las tres variables de flujo: velocidad, densidad y velocidad de flujo.

\subsubsection{Datos de entrada requeridos}

El análisis de una autopista de segmentos básicos requiere información relativa de características geométricas del segmento y las características de la demanda de los usuarios. Esta sección presenta los datos de entrada necesarios para la metodología de las autopistas de segmentos básicos; en esta parte se dan detalles acerca de los parámetros individuales que se necesitan en la metodología.

Datos para Autopistas.

Se necesita la siguiente información sobre las características geométricas del segmento para realizar un análisis (se muestran los rangos típicos de estos parámetros):

1. FFS: 55 a $75 \mathrm{mi} / \mathrm{h}$

2. Número de carriles de la autopista sin peaje de la línea principal (en una dirección): al menos dos.

3. Ancho de carril: 3 metros a 3.60 metros.

4. El lado derecho de un espacio lateral: 0 metros a más de 1.8 metros.

5. Total de densidad rampa: 0 a 3 rampas / $\mathrm{km}$

Datos para la demanda. 
Se requiere la siguiente información sobre los usuarios del segmento:

1. La demanda a la hora del análisis o la demanda diaria y $\mathrm{K}$ y D-factores;

2. Presencia (proporción de camiones, autobuses y vehículos recreativos) de vehículos pesados: 0 a $100 \%$ en terreno en general, o de 0 a $25 \%$ o más para los grados específicos.

3. Factor de horas pico (PHF): hasta 1,00

4. Factor de Población que conduce: 0,85 y 1,00.

5. El período de análisis para cualquier autopista es generalmente de $15 \mathrm{~min}$ en la hora pico.

\subsection{TEORIA DE REVERSIBILIDAD}

Los carriles reversibles en vías saturadas son uno de los mecanismos para aliviar el congestionamiento en horas pico, esta es una actividad de gestión del tráfico. Los carriles reversibles proporcionan una capacidad adicional para los flujos máximos direccionales dependiendo de la hora del día. Los carriles reversibles en las carreteras pueden estar situados en el centro de una autopista bajo un control de puerta en ambos extremos. Estos carriles surgen por una instalación de flujo ininterrumpido en la vía, estos pueden ser implementados para equilibrar los niveles de servicio que se tienen en las vías de ida y retorno con una ayuda del control de carril tanto como el uso de señales y signos que se pueden abrir y cerrar carriles por sentido, en horas de mayor congestionamiento vehicular.

El uso temporal de los carriles reversibles durante las horas pico pueden proporcionar una capacidad adicional en una sección de cuello de botella y mejorar el rendimiento general del nivel de servicio de esa vía. A su vez el uso temporal de estos carriles reversibles puede mejorar los tiempos de transitividad de los vehículos de transporte público ubicados en la cola por lo que les permite llegar a su salida sin tener que esperar en la cola de la línea principal.

Para optar estas medidas de gestión del tráfico (carriles reversibles), se evalúa lo siguiente:

- Análisis de flujo de tráfico.

- Análisis de volumen.

- Análisis de capacidad. 


\subsection{TEORIA DE AFOROS VEHICULARES}

Se denomina aforo al proceso de medir la cantidad de vehículos que pasan por un tramo en una carretera en una unidad de tiempo, existen diversas formas de obtener los conteos de los volúmenes de tránsito. Según Cal y Mayor en su 8va edición:

“(...) los aforos manuales a cargo de personas, los cuales son particularmente útiles para conocer el volumen de los movimientos direccionales en intersecciones, los volúmenes por carriles individuales y la composición vehicular. Los aforos por combinación de métodos manuales y mecánicos, tales como el uso de contadores mecánicos accionados manualmente por observadores. Los aforos con el uso de dispositivos mecánicos, los cuales automáticamente contabilizan y registran los ejes de los vehículos. Y los aforos con la utilización de técnicas tan sofisticadas como las cámaras fotográficas, las filmaciones y los equipos electrónicos adaptados a computadoras."

Las razones para efectuar los aforos son muy variables, para nuestro caso de estudio mencionaremos por ejemplo las siguientes razones para aforo vehicular:

Determinar el Trafico Promedio Diario (TPD), que es el promedio de 24 horas de conteo efectuados en un número de días mayor a 1 pero menor a 1 año. El TPD se puede utilizar para:

- Planeamiento de las actividades de la carretera.

- Medición de la demanda actual.

- Evaluación del flujo de tráfico existente.

Determinar el Volumen Horario de Máxima Demanda (VHMD), que es el número máximo de vehículos que pasan por un tramo de carretera durante un periodo de 60 minutos consecutivos. El VHMD se utiliza para:

- Clasificaciones funcionales de las carreteras.

- Diseño de las características geométricas de la carretera, por ejemplo, número de carriles, señalización de intersecciones o canalización.

- Análisis de la capacidad.

- Desarrollo de programas relacionados con las operaciones del tráfico, por ejemplo, sistemas de una calle unidireccional o el encaminamiento del tráfico. 
- Desarrollo de las regulaciones del estacionamiento.

- Evaluación de los niveles de servicio en la vía a estudiar.

Determinar la Clasificación Vehicular (CV), que registra el volumen con respecto al tipo de vehículos, por ejemplo, automóviles de pasajeros, automóviles de 2 ejes, automóviles de 3 ejes. La CV se utiliza en:

- Diseño de características geométricas, con particular referencia a los requerimientos de radios de giro, pendientes máximas, anchos de carril.

- Análisis de la capacidad, con respecto a los pasajeros de los automóviles.

- Ajuste de los conteos de tráfico obtenidos por maquinas.

- Diseño estructural de pavimentos de la carretera, puentes, etc.

Determinar los Kilómetros Recorridos del Vehículo (KRV), es una medida del recorrido a lo largo de una sección del camino. Es el producto del volumen de tráfico (es decir, el volumen medio del día laborable o TPD) y de la longitud del camino, en los kilómetros a los cuales el volumen es aplicable. KRV se utiliza principalmente como base para asignar los recursos para el mantenimiento y la mejora de carreteras

\subsection{FACTORES DE CONVERSIÓN A UNIDAD DE COCHE PATRÓN (UCP)}

En la tabla 1 se muestra los factores que son usados para uniformizar los tamaños de los vehículos a un solo tipo de vehículos patrón, al cual hay que transformar por equivalencia los demás tipos de vehículos. Esta unidad representativa de todos los modos de transporte se denomina UCP, cuya equivalencia es igual a la unidad. A efectos de uniformizar el registro de datos de los aforos vehiculares para los modos de transporte público y privado, se utilizaron factores de conversión a UCP. 
Tabla 1: Equivalencia en UCP

\begin{tabular}{|c|c|}
\hline TIPO DE VEHICULOS & FACTOR UCP \\
\hline AUTO & 1 \\
\hline BUS & 3 \\
\hline MICROBUS & 2 \\
\hline CAM. RURAL & 2 \\
\hline CAMION PESADO & 2.5 \\
\hline OMNIBUS INTERPROV. & 3.5 \\
\hline
\end{tabular}

Fuente GTU-AATE

\subsection{DISPOSITIVOS DE CONTROL VEHICULAR}

En los dispositivos de control se usarán una combinación de señales de uso estático, uso dinámico de carriles y marcas de pavimento. Usando una combinación de estos dispositivos de control de tráfico, a los automovilistas se les dirá qué carriles están abiertos o cerrados para su dirección de viaje.

\subsubsection{Balizar conos de transito:}

En la Imagen 17 se muestran elementos que sirven para la señalización vial y representan un elemento de seguridad para conductores, sirven para indicar desvíos en la carretera.

Imagen 17: Balizaje de conos

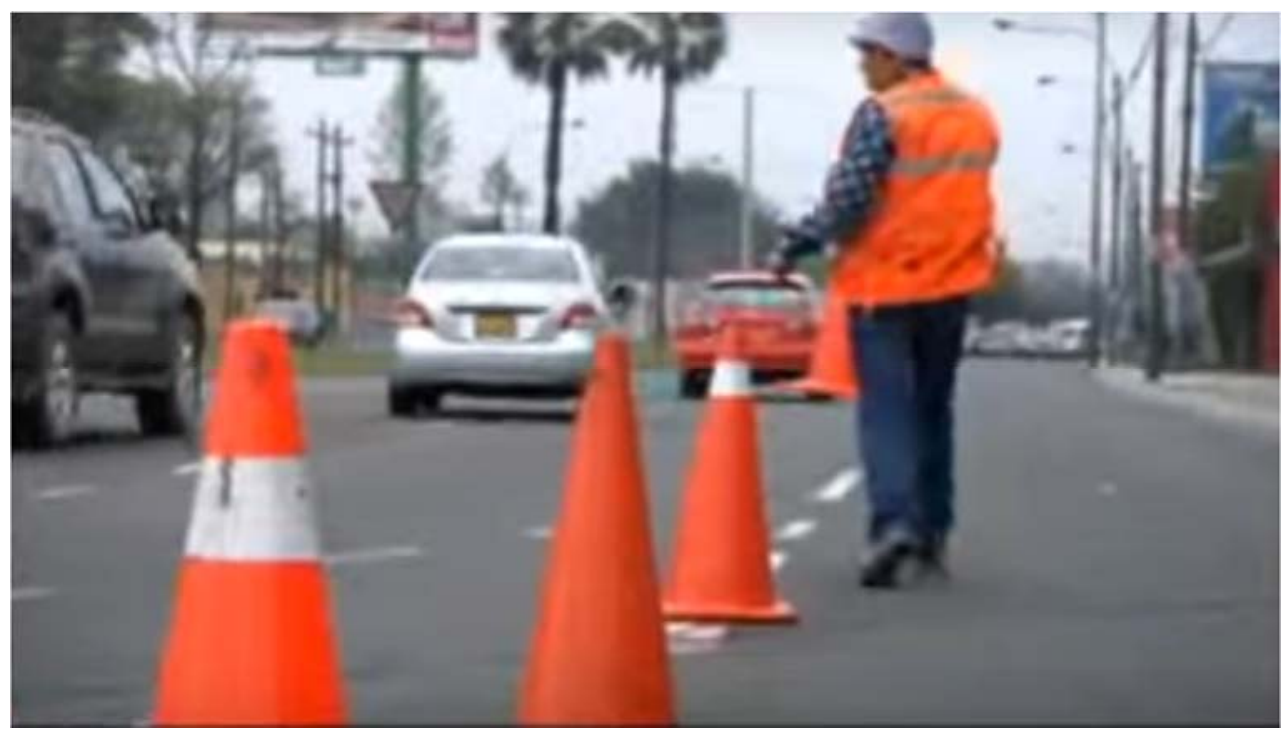

Fuente: Propia 


\subsubsection{Señalización semafórica:}

La señalización aérea reduce el número de conos y barriles a lo largo de las calles. Estos se pueden ajustar para convertir un carril diseñado inicialmente en un sentido en sentido contrario, en hora pico. Como norma general, la utilización de los carriles reversibles viene determinada por los semáforos de ocupación de carril que suelen estar colocados sobre pórticos (Como en la imagen 17), pasos elevados, etc., y afectan exclusivamente a los vehículos que circulen por el carril sobre el que están situados dichos semáforos.

Una luz roja en forma de aspa indica la prohibición de ocupar el carril sobre el que se encuentra encendida a aquellos vehículos hacia los que esté dirigida la luz. Los conductores deberán abandonarlo en el tiempo más breve posible, porque ese carril está dispuesto para ser utilizado en sentido contrario.

Una luz verde en forma de flecha apuntada hacia abajo indica que está permitido circular por el carril sobre el que está encendida.

Una luz blanca o amarilla, intermitente o fija, en forma de flecha, no incorporada a una señal de orientación, colocada encima de un carril y apuntando hacia abajo en forma oblicua, indica la necesidad de irse incorporando, en condiciones de seguridad, hacia el carril al que apunte la flecha, porque el carril por el que se circula va a quedar cerrado en corto espacio.

Imagen 18: Semaforización aérea

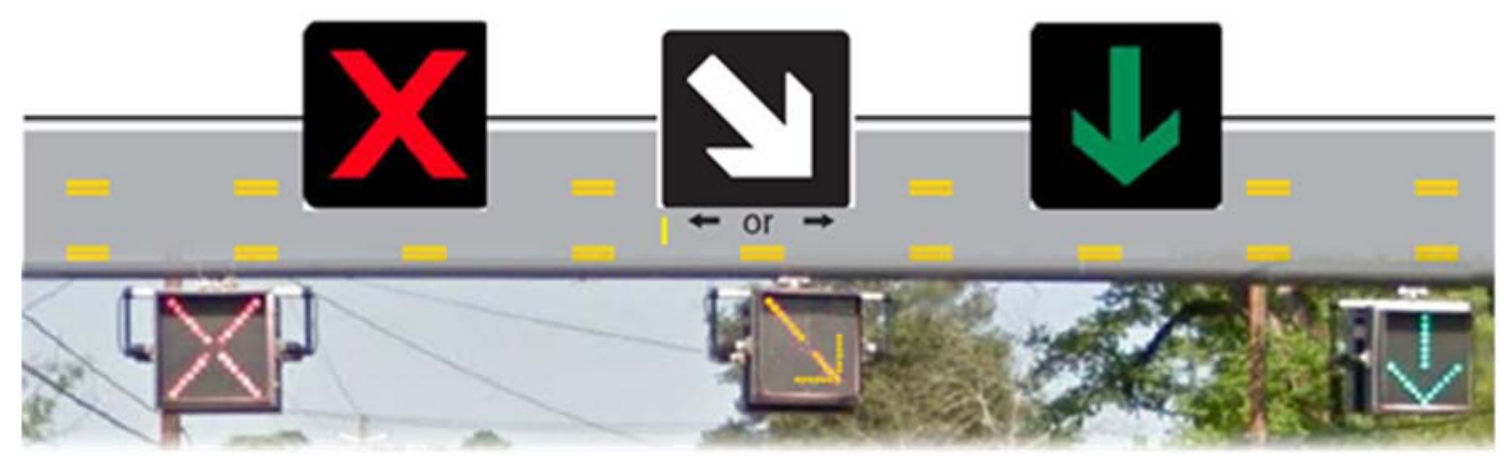

Fuente: Revista Conducir Mejor .Madrid-2000

\subsubsection{Marcas en el pavimento:}

Las marcas en el pavimento dentro de la zona de transición consisten en redireccionar a los conductores hacia los carriles reversibles con una flecha diagonal para dirigirlos al 
inicio o fin de estos carriles como se indican en la Imagen 18. El proceso de transición es muy similar al proceso de iniciación. La zona de transición de un bloque comienza con un signo que aconseja al conductor que está circulando por un carril reversible, tal como se indica en la Imagen 19.

Imagen 19: Inicio de carril reversible

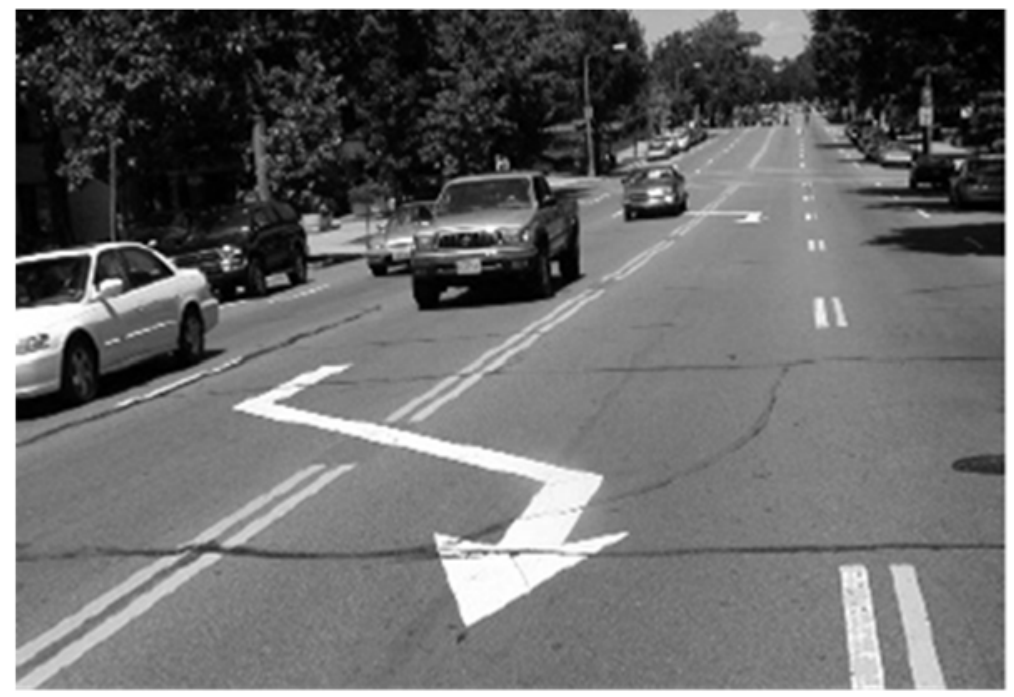

Fuente: NCHRP Synthesis 340 - Convertible Roadway and lanes

Imagen 20: Tramo de transición de carril reversible

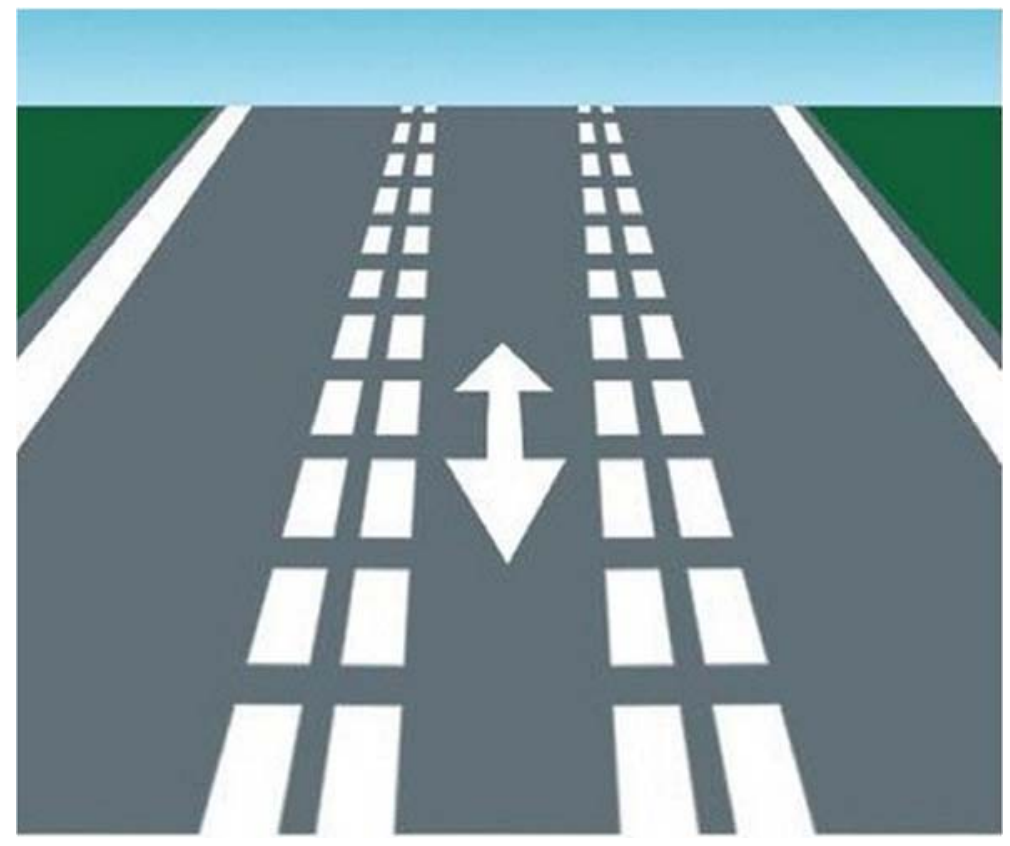

Fuente: Autoescuela - Carril reversible 


\subsubsection{Pantallas de mensajes variables:}

Los paneles de mensaje variable (LED) son elementos fundamentales de los sistemas de gestión dinámica de tráfico. En la Imagen 20, según las condiciones del tráfico, la pantalla variable informa, advierte y guía a los automovilistas en carreteras y autopistas. Los paneles de mensajes variables pueden colocarse en multitud de lugares como carreteras, principales cruces de carreteras y arterias urbanas. Se suelen instalar a un lado o encima de la carretera y utilizan texto y gráficos monocromos o en color

Imagen 21: Panel aéreo

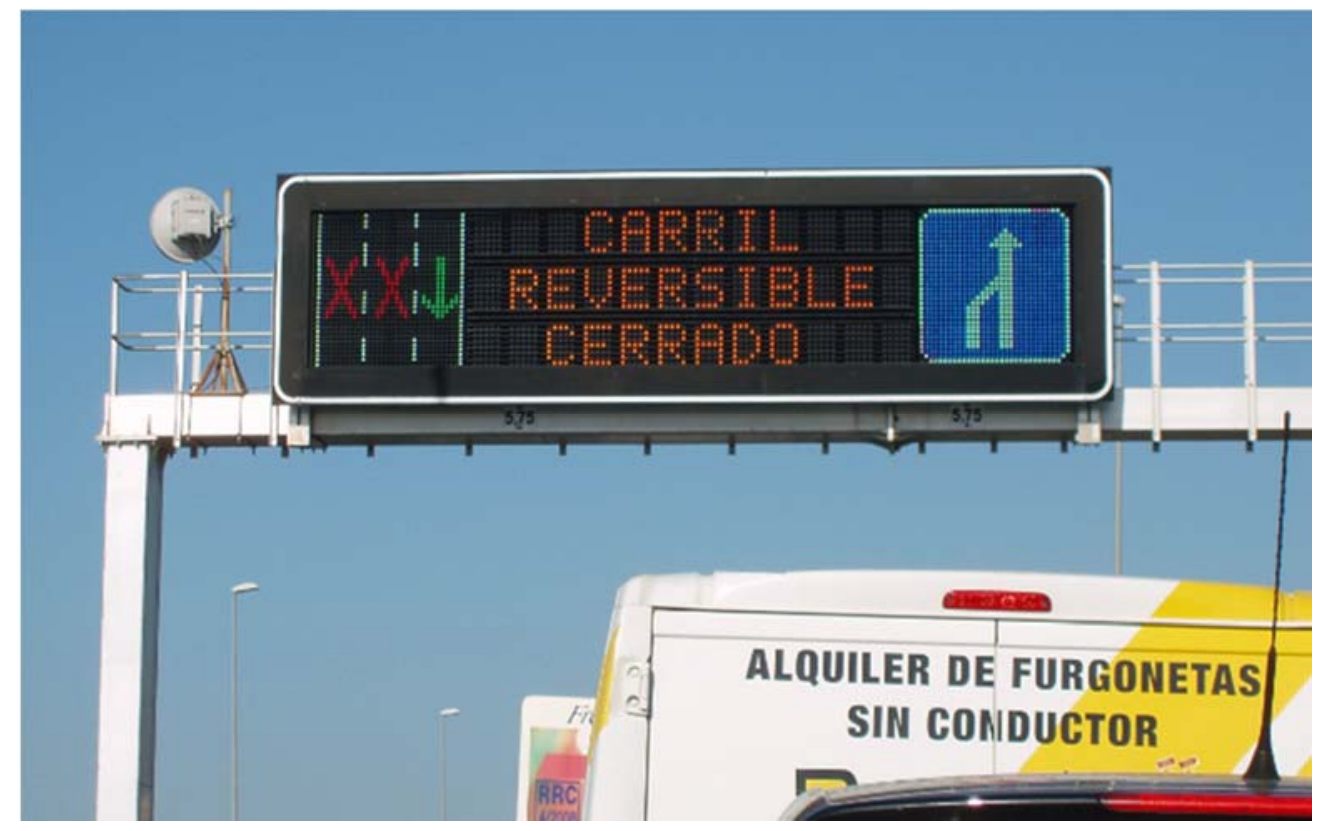

Fuente: Ayuntamiento de Madrid)

\subsection{ESTUDIO DE IMPACTO VIAL}

Este estudio prioriza la gestión de la demanda regulando el uso de los sistemas existentes, y así intervenir en el comportamiento social beneficiando a la ciudad y sus habitantes, en base a este contexto, los municipios son la entidad responsable de la planificación y gestión urbana, por ende, es su responsabilidad exigir la realización de los Estudios de Impacto Vial (EIV) cuando una vía se encuentra en posible estado de congestionamiento. Asimismo, es de suma importancia que la entidad responsable defina con claridad cuando se hace necesario la realización de un EIV y cuál será el contenido del mismo. Dichos planteamientos y requerimientos solicitados deben ser cumplidos al pie de la letra con el 
fin de lograr el objetivo fundamental del estudio, el cual es evaluar los impactos que se van a producir sobre la red vial de transporte.

Hoy en día, existen problemas de congestión vehicular en casi todas las calles de Lima Metropolitana, al no tener un adecuado Sistema de Control de Transporte y Crecimiento Urbano nuestra capital está sufriendo los problemas de congestión vial.

Es así, que en los dos últimos mandatos distritales y Municipal de Lima Metropolitana está tomando énfasis en mejorar el Sistema de Control de Transporte Urbano con programas de desarrollo urbano y nuevas ordenanzas municipales.

Según la ordenanza $\mathrm{N}^{\circ} 1268$, emitida el 2 de julio de 2009 por la MUNICIPALIDAD METROPOLITANA de LIMA, en el artículo $\mathrm{N}^{\circ} 3$, define a un ESTUDIO de IMPACTO VIAL (EIV) como:

"el conjunto de actividades que permiten evaluar cualitativa y cuantitativamente los efectos que produce sobre el entorno vial y transporte, el desarrollo urbanístico o el proceso de renovación de zonas o lotes de terreno, de forma de poder prever y mitigar sus efectos negativos mediante medidas administrativas y técnicas adecuadas, de manera que sea posible recuperar, alcanzar o mejorar el nivel de servicio existente en el entorno" $"$

Además, la Municipalidad de Lima Metropolitana está priorizando el estudio de este impacto vial en cada nuevo proyecto de construcción u obra civil, para gestionar la demanda y regular la oferta vehicular que implica la construcción del nuevo proyecto y con ello favorecer a los ciudadanos.

\subsection{FUNCIONALIDADES DEL AIMSUN}

Llevando la integración un paso más allá, este simulador híbrido proporciona simulaciones microscópicas y mesoscópicas simultáneas, lo que permite simular grandes áreas para luego acercarse en las áreas que requieren un mayor nivel de detalle.

La unión de un modelo mesoscópico basado en sucesos con un microsimulador más detallado segmentado temporalmente ofrece un escenario que recoge lo mejor de ambos

${ }^{3}$ Cfr. ORDENANZA Nº1268 - 2009 - MUNICIPALIDAD METROPOLITANA DE LIMA. 
mundos, combinando una eficiencia computacional muy elevada con una representación precisa de las dinámicas de tráfico.

Consolidar la simulación de tráfico en una única aplicación multifunción y multicapa proporciona los siguientes beneficios:

- Capacidad de escoger la herramienta más adecuada para cada proyecto en vez de adaptar los proyectos a las herramientas.

- Desarrollo de modelos de simulación extensibles a una escala cada vez mayor utilizando un único paquete integrado.

- Desaparición de la transferencia manual entre diferentes paquetes de software macroscópicos, mesoscópicos y microscópicos y de los errores derivados.

- Eliminación del trabajo redundante que supone mantener diferentes redes en diversos paquetes de software.

- Reducción de los costes administrativos y de mantenimiento, así como del soporte necesario por parte de expertos.

Una de las funcionalidades más destacadas de Aimsun es su velocidad: este microsimulador es, con diferencia, el más rápido del mercado y la herramienta de simulación de más calidad disponible a nivel mundial para proyectos de gran escala.

La arquitectura multi hilo de Aimsun acelera la velocidad del proceso de simulación hasta un punto en el que se redefine completamente lo que se puede incluir en un modelo dinámico, ya sea una gran ciudad o una red de autopistas muy grande y compleja.

Este proyecto presentará la nueva arquitectura con carril reversible del caso de estudio del sistema implementada en Aimsun con especial énfasis en la consistencia del modelo mesoscópico y pretende demostrar cómo esta consistencia se complementa utilizando resultados computacionales en una red de gran tamaño.

Incluso en un portátil, el microsimulador de Aimsun puede simular un modelo de la ciudad de Singapur con 10.580 intersecciones y $4.483 \mathrm{~km}$ de carriles de 2 a 3 veces más rápido que en tiempo real. La simulación a gran velocidad permite: 
- Simular áreas más amplias sin dejar de lado posibles zonas problemáticas.

- Probar un mayor número de escenarios en el tiempo disponible, lo que permite un análisis de supuestos más amplio para una gran diversidad de condiciones de tráfico.

- Manejar situaciones en las que el tiempo de resolución es crítico.

Para la Construcción del sistema:

- Secciones y señalizaciones

- Tipos de vehículos

- Paraderos de transporte publico

- Controles de acceso

Datos de entrada:

- Datos de tráfico en una matriz $\mathrm{O} / \mathrm{D}$

- Condiciones de trafico

Resultados:

- Generar resultados visuales con simulaciones

- Tablas de resultados: Densidades. Niveles de servicio, Tiempo de recorrido y velocidades.

- Reordenamiento del tráfico. 


\section{CAPITULO III: METODOLOGÍA}

\subsection{AFOROS VEHICULARES PARA EL CASO DE ESTUDIO}

El estudio para el aforo vehicular constará de tres etapas:

1. Planificación

2. Toma de información

3. Trabajos de gabinete

Planificación

Se planificó realizar un conteo clasificado de vehículos, los días típicos Martes y Jueves de una semana entre los meses de Noviembre, Febrero y Abril, cuyos intervalos de hora fueron de 6:00 - 11:00 / 12:00 - 17:00 / 18:00 - 22:00 teniendo un total de 14 horas al día, cerrando la información cada cuarto de hora, para determinar la hora pico y el cuarto de hora pico. Los conteos se realizarán de acuerdo a cada intersección.

Las ubicaciones de las estaciones de conteo serán en las siguientes:

- Panamericana Norte con el puente peatonal Control Zarumilla.

- Panamericana Norte con intercambio vial Tomas Valle

Toma de Información

Los conteos y clasificación del tráfico se realizaron con personal los cuales fueron capacitados para el reconocimiento del tipo de vehículos y manejo de contadores manuales registrando cada movimiento de la intersección. Los formatos empleados son los que se han usado en otros estudios realizados por el Ministerio de Transporte y Comunicación y se presentarán en el Anexo 1.

La clasificación de los vehículos fue la siguiente:

- Autos y camionetas 
- Camionetas Rurales

- Ómnibus y buses.

- Camiones de 2 y 3 ejes

- Camiones de 4 y 5 ejes.

- Camiones de 6 ejes.

Trabajo de Gabinete

En base a los conteos y clasificación vehicular realizada en el campo se confeccionaron cuadros y flujogramas para cada intersección en horas pico de la mañana y de la tarde.

\subsection{SEGMENTO DE AUTOPISTA BÁSICA}

Para nuestro caso de estudio, esta metodología se puede utilizar para analizar la capacidad, los niveles de servicios, lo requisitos del carril y los efectos de las características de diseño en el rendimiento del segmento de autopista básica. La metodología se basa en los resultados elaborados por un artículo presentado por la National Cooperative Highway Research Program (NCHRP), que ha sido parcialmente actualizado.

Limitaciones de la metodología:

La metodología de este capítulo no se aplica en lo siguiente:

- Carriles especiales reservados para un solo tipo de, carriles de camiones y carriles de ascenso.

- Líneas de Control (para restringir el cambio de carril).

- Puentes y túneles con segmentos de ampliación.

- Segmentos cerca de una zona de peaje.

- Instalaciones con velocidad de flujo libre menor de $55 \mathrm{mi} / \mathrm{h}$ y más de $75 \mathrm{mi} / \mathrm{h}$.

- Límite de velocidad y de aplicación de las prácticas.

- Presencia de sistemas de transporte inteligentes (ITS) relacionada con vehículos u orientadas al conductor. 
- Capacidad para mejorar los efectos de medición de rampas.

- Efectos operacionales de las condiciones sobresaturadas.

En la mayoría de los casos que acabamos de citar, se tendrá que utilizar otras alternativas de herramientas o recurrir a otra información de la investigación y el desarrollo para fines especiales en modificaciones de esta metodología para incorporar los efectos de cualquiera condición mencionada.

\subsubsection{Descripción y procedimiento de la metodología}

La metodología de este capítulo sirve para el análisis de un segmento de autopista básica. En la Imagen 22, tenemos un flujo grama de la metodología utilizada en el análisis operativo. Esta metodología también se puede aplicar directamente para determinar el número de carriles requerido para proporcionar un Nivel de Servicio para un volumen proporcionado de demanda.

Imagen 22: Flujograma del Segmento de Autopista Básica

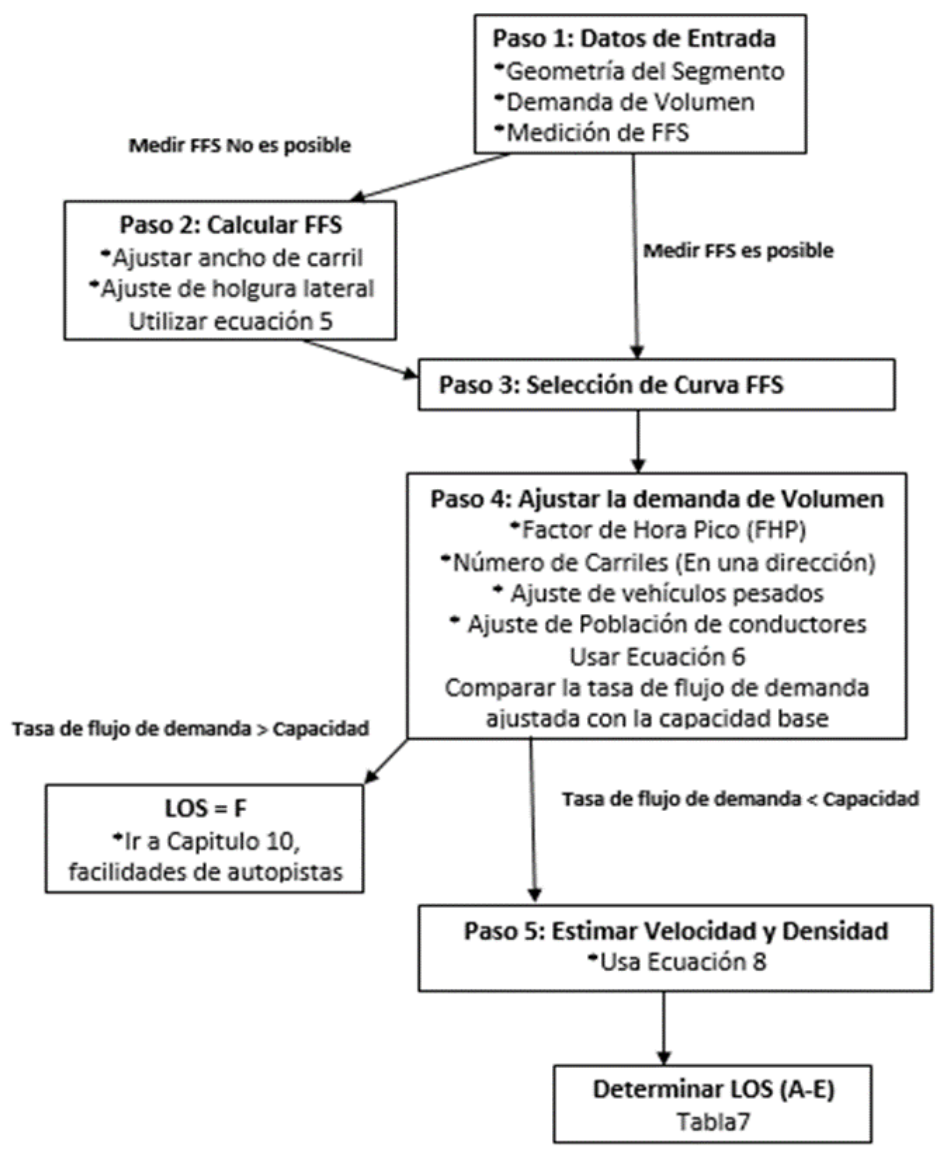

Fuente: HCM 2010 
Pasos para el cálculo

Paso 1: Datos de Entrada

Para un análisis operativo, como se señaló anteriormente, se tiene que especificar (bien con valores específicos del sitio o por defecto) la demanda de volumen, número y ancho de las vías, espacio lateral del lado derecho, la densidad total de rampa, porcentaje de los vehículos pesados (camiones, autobuses, tráileres), FHP (Factor de hora pico) y el factor de población que conduce.

Paso 2: Calcular la velocidad de Flujo Libre (FFS)

La velocidad de Flujo Libre (FFS) se puede determinar directamente a partir de mediciones de campo o se pueden estimar como se describe a continuación:

Campo de medición de la velocidad de flujo libre.

La velocidad de flujo libre (FFS) es la velocidad media de pasajeros - son los vehículos durante los períodos de bajo flujo moderado. (Hasta $1.000 \mathrm{veh} / \mathrm{h} / \mathrm{c}$ ). Para un segmento específico de la autopista, las medias velocidades son prácticamente constante en esta gama de velocidades de flujo. Si el FFS es medido directamente, se aplicará al valor medido sin ajustes.

El estudio de velocidad debe realizarse en una ubicación que es representativa del segmento en un momento adecuado cuando las tasas de flujo son menores a $1.000 \mathrm{veh} / \mathrm{h} / \mathrm{c}$. La velocidad de estudio debe medir la de todos los vehículos). Se debe obtener una muestra de al menos 100 vehículos.

Estimación de la velocidad de flujo libre.

En este caso, la velocidad de flujo libre (FFS) del segmento puede estimarse utilizando la ecuación, que se basa en las características físicas del segmento en estudio:

$$
\mathrm{FFS}=75.4-f_{\mathrm{LW}}-f_{\mathrm{LC}}-3.22 \mathrm{TRD}^{0.84}(\text { Ecu. 5) }
$$

Dónde:

FFS = Velocidad de flujo libre del segmento básico de la autopista (mi/h). 
$f_{\mathrm{LW}}=$ ajuste de ancho de carril $(\mathrm{mi} / \mathrm{h})$,

$f_{\mathrm{LC}}=$ ajuste por espacio lateral derecho $(\mathrm{mi} / \mathrm{h})$

$\mathrm{TRD}=$ densidad total de la rampa $(\mathrm{rampa} / \mathrm{mi})$.

Una vez obtenido el FFS con la unidad $\mathrm{mi} / \mathrm{h}$, convertirlo a $\mathrm{km} / \mathrm{h}$ para nuestro caso de estudio.

Base de las velocidades de flujo libre (FFS)

Esta metodología abarca segmentos básicos de la autopista con la FFS que van desde 80 $\mathrm{km} / \mathrm{h}$ a $113 \mathrm{~km} / \mathrm{h}$. Por lo tanto, el algoritmo de predicción debe comenzar con una velocidad base de $113 \mathrm{~km} / \mathrm{h}$ o superior. Se optó por un valor de $115 \mathrm{~km} / \mathrm{h}$, ya que dio lugar a la mayor parte Predicciones precisas frente a los datos recogidos en un anterior estudio.

Ajuste por ancho de carril (flw)

La condición base para el ancho de carril es de 3.6 metros o más. Cuando el carril de la media anchura a través de todos los carriles es menos de 3.6 metros, el FFS se ve afectada negativamente.

Los ajustes para reflejar el promedio del efecto de la anchura del carril más estrecho se muestran en la tabla 2.

Tabla 1: Ajuste de ancho de carril en relación al FFS

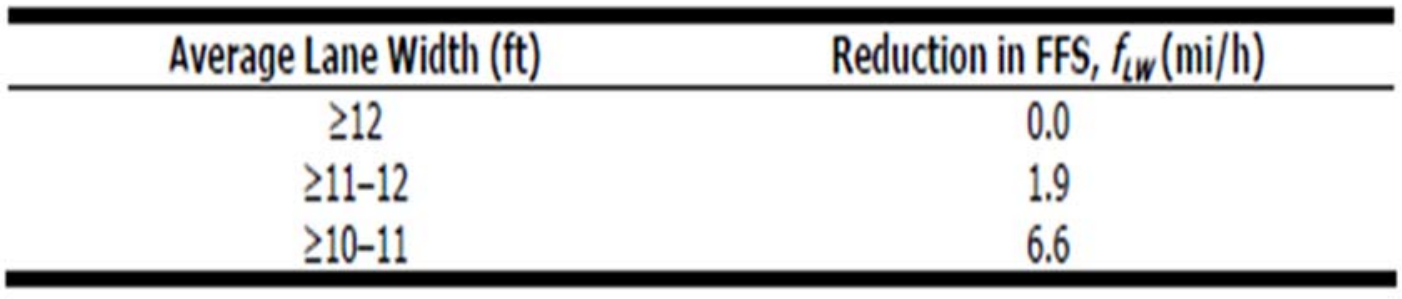

Fuente: HCM 2010

Ajuste por espacio lateral (flc)

La condición base para el despeje lateral del lado derecho es de 6 pies o más. Los despejes de un espacio lateral se miden desde el borde derecho del carril de circulación hacia la 
más cercana obstrucción lateral. Se debe tener cuidado para identificar una "obstrucción lateral." Algunas obstrucciones pueden ser continuas, tales como muros de contención, barreras de hormigón o barandillas. Otros pueden ser periódica, tales como soportes de luz o estribos de puentes. La tabla 3 muestra las adaptaciones de las velocidades de flujo libre (FFS) de base debido a la existencia de obstrucciones a menos de $1.80 \mathrm{~m}$ hasta el borde derecho del carril de circulación.

Tabla 2: Adaptación del FFS debido a la obstrucción del carril

\begin{tabular}{c|cccc}
\hline $\begin{array}{c}\text { Right-Side } \\
\text { Lateral } \\
\text { Clearance (ft) }\end{array}$ & $\mathbf{2}$ & \multicolumn{4}{c}{ Lanes in One Direction } & \\
\hline$\geq 6$ & 0.0 & 0.0 & 4 & $\geq 5$ \\
\hline 5 & 0.6 & 0.4 & 0.0 & 0.0 \\
4 & 1.2 & 0.8 & 0.2 & 0.1 \\
3 & 1.8 & 1.2 & 0.4 & 0.2 \\
2 & 2.4 & 1.6 & 0.6 & 0.3 \\
1 & 3.0 & 2.0 & 0.8 & 0.4 \\
0 & 3.6 & 2.4 & 1.0 & 0.5 \\
\hline
\end{tabular}

Fuente: HCM 2010

La densidad total de rampa (TRD)

La Ecuación anterior incluye un término que da cuenta a los efectos del total de rampas para la velocidad de flujo continuo. La densidad total de rampa se define como el número de rampas situado entre el punto medio del segmento autopista básica.

Paso 3: Selección de la curva de la velocidad de flujo libre (FFS)

Como se señaló anteriormentge, una vez que se determina la autopista de segmento básica, una de las cinco curvas de velocidad de flujo básico (Imagen Curvs) se selecciona para utilizarlo en el análisis. No se recomienda la interpolación entre las curvas.

Paso 4: Ajuste del volumen de la demanda

Dado que las curvas de velocidad de flujo básico de la Ecuación 6 se basan en las tasas de flujo en pasajeros equivalentes por hora, con la población de conductores dominados por usuarios habituales del segmento de autopista básica, los volúmenes de demanda expresan como los vehículos por hora en las condiciones existentes deben ser convertidos a esta base: 


$$
\mathrm{v}=\frac{\mathrm{V}}{\text { PHF } \times \mathrm{N} \times \mathrm{fHV} \times f \mathrm{p}}(\text { Ecu. } 6)
$$

Dónde:

$v p=$ tasa de flujo de demanda en condiciones equivalentes de base $(v e h / h / c)$.

$\mathrm{V}=$ volumen de la demanda en las condiciones prevalecientes (veh/h).

$\mathrm{FHP}=$ factor de hora pico

$\mathrm{N}=$ número de carriles en la dirección de análisis.

$\mathrm{fHV}=$ factor de ajuste para la presencia de vehículos pesados en el flujo de tráfico.

$\mathrm{fp}=$ factor de ajuste para las poblaciones de controladores que no conoce.

Factor Hora Pico (PHF)

El FHP representa la variación en el flujo de tráfico dentro de una hora. Observaciones del flujo de tráfico indican consistentemente que las velocidades de flujo encontradas en el pico de 15 min dentro de una hora, no se sostienen a lo largo de toda la hora. En las autopistas, el FHP típicos oscilan 0,85 a 0,98. Los valores más bajos dentro de ese rango son típicos de condiciones de menor volumen. Los valores más altos dentro de esa gama son típicos de las condiciones urbanas y suburbanas en las horas pico. Los datos de campo a ser utilizado deben reflejar si es posible las condiciones locales que representa este factor.

El ajuste para vehículos pesados. (fHv)

Un vehículo pesado se define como cualquier vehículo con más de cuatro ruedas en el pavimento durante su funcionamiento normal. Dichos vehículos son generalmente clasificados como camiones, autobuses, vehículos recreativos. Estos cubren una amplia variedad de vehículos, desde una sola unidad, camiones con neumáticos, traseros dobles a unidades triples, combinaciones de tractor-remolque, autobuses interurbanos, autobuses de transporte público y privado. Porque los autobuses son en muchos aspectos similares a los camiones unitarios, ambos tipos de los vehículos son considerados en una categoría.

El factor de ajuste de vehículos pesados fHV se calcula como sigue: 


$$
f h v=\frac{1}{1+P t(E t-1)+\operatorname{Pr}(E r-1)}(\text { Ecu. 7) }
$$

Dónde:

$\mathrm{f}_{\mathrm{HV}}=$ Factor de ajuste de vehículos pesados.

$\mathrm{P}_{\mathrm{T}}=$ Proporción de camiones y autobuses en el flujo de tráfico.

$\mathrm{P}_{\mathrm{R}}=$ Proporción de vehículos recreativos en el flujo de tráfico.

$\mathrm{E}_{\mathrm{T}}=$ Coche de pasajeros equivalente $(\mathrm{PCE})$ de un camión o un autobús en el flujo de tráfico.

$E_{R}=P C E$ de un RV en el flujo de tráfico.

El factor de ajuste se encuentra en un proceso de dos pasos. En primer lugar, el Coche de Pasajero Equivalente (PCE) para cada camión o autobús y RV se encontraron para las condiciones prevalecientes en estudio. Estos valores de la equivalencia representan el número de vehículos de pasajeros que utilizarían la misma cantidad de capacidad de la autopista como un camión o autobús o RV bajo las prevalecientes condiciones. En segundo lugar, la tabla se utiliza para convertir los valores de PCE al factor de ajuste. En la tabla 4 se muestra el PCE para camiones, autobuses y vehículos recreativos en terrenos de segmentos generales.

Tabla 3: PCE para camiones, autobuses y vehículos recreativos

\begin{tabular}{c|ccc}
\hline & & \multicolumn{3}{|c}{ PCE by Type of Terrain } \\
Vehicle & Level & Rolling & Mountainous \\
\hline Trucks and buses, $E_{T}$ & 1.5 & 2.5 & 4.5 \\
RVs, $E_{R}$ & 1.2 & 2.0 & 4.0 \\
\hline
\end{tabular}

Fuente: HCM 2010

\section{Equivalentes para las actualizaciones específicas}

Las tablas 5 y 6, dan valores de ET para camiones y autobuses y ER para vehículos recreativos, respectivamente. Estos factores varían de acuerdo con el porcentaje de grado, antigüedad grado, y la proporción de vehículos pesados en la corriente de tráfico. Máximo Los valores se presentan cuando hay sólo unos pocos vehículos pesados en el flujo de 
tráfico. Los equivalentes disminuyen a medida que el número de vehículos pesados aumenta debido a que estos vehículos tienden a formar pelotones. Debido a que los vehículos pesados tienen más uniforme Características de funcionamiento, un menor número de grandes huecos se crean en el flujo de tráfico y el impacto de un vehículo pesado en un pelotón es menos grave que la de un solo vehículo pesado en una corriente de coches de pasajeros principalmente. En los grados de material compuesto, la inclinación relativa de los segmentos es importante. Si una actualización de un 5\% es seguido por una actualización de $2 \%$, por ejemplo, el impacto máximo es más probable en el extremo del segmento $5 \%$ en vehículos pesados. Los vehículos pesados se espera que se acelere después de entrar en el segmento de $2 \%$.

Tabla 5: Ajuste de volumen de la demanda para vehículos pesados.

\begin{tabular}{|c|c|c|c|c|c|c|c|c|c|c|}
\hline \multirow{2}{*}{$\begin{array}{c}\text { Upgrade } \\
(\%)\end{array}$} & \multirow{2}{*}{$\begin{array}{c}\text { Length } \\
\text { (mi) }\end{array}$} & \multicolumn{9}{|c|}{ Proportion of Trucks and Buses } \\
\hline & & $2 \%$ & $4 \%$ & $5 \%$ & $6 \%$ & $8 \%$ & $10 \%$ & $15 \%$ & $20 \%$ & $\geq 25 \%$ \\
\hline$\leq 2$ & All & 1.5 & 1.5 & 1.5 & 1.5 & 1.5 & 1.5 & 1.5 & 1.5 & 1.5 \\
\hline \multirow{6}{*}{$>2-3$} & $0.00-0.25$ & 1.5 & 1.5 & 1.5 & 1.5 & 1.5 & 1.5 & 1.5 & 1.5 & 1.5 \\
\hline & $>0.25-0.50$ & 1.5 & 1.5 & 1.5 & 1.5 & 1.5 & 1.5 & 1.5 & 1.5 & 1.5 \\
\hline & $>0.50-0.75$ & 1.5 & 1.5 & 1.5 & 1.5 & 1.5 & 1.5 & 1.5 & 1.5 & 1.5 \\
\hline & $>0.75-1.00$ & 2.0 & 2.0 & 2.0 & 2.0 & 1.5 & 1.5 & 1.5 & 1.5 & 1.5 \\
\hline & $>1.00-1.50$ & 2.5 & 2.5 & 2.5 & 2.5 & 2.0 & 2.0 & 2.0 & 2.0 & 2.0 \\
\hline & $>1.50$ & 3.0 & 3.0 & 2.5 & 2.5 & 2.0 & 2.0 & 2.0 & 2.0 & 2.0 \\
\hline \multirow{6}{*}{$>3-4$} & $0.00-0.25$ & 1.5 & 1.5 & 1.5 & 1.5 & 1.5 & 1.5 & 1.5 & 1.5 & 1.5 \\
\hline & $>0.25-0.50$ & 2.0 & 2.0 & 2.0 & 2.0 & 2.0 & 2.0 & 1.5 & 1.5 & 1.5 \\
\hline & $>0.50-0.75$ & 2.5 & 2.5 & 2.0 & 2.0 & 2.0 & 2.0 & 2.0 & 2.0 & 2.0 \\
\hline & $>0.75-1.00$ & 3.0 & 3.0 & 2.5 & 2.5 & 2.5 & 2.5 & 2.0 & 2.0 & 2.0 \\
\hline & $>1.00-1.50$ & 3.5 & 3.5 & 3.0 & 3.0 & 3.0 & 3.0 & 2.5 & 2.5 & 2.5 \\
\hline & $>1.50$ & 4.0 & 3.5 & 3.0 & 3.0 & 3.0 & 3.0 & 2.5 & 2.5 & 2.5 \\
\hline \multirow{5}{*}{$>4-5$} & $0.00-0.25$ & 1.5 & 1.5 & 1.5 & 1.5 & 1.5 & 1.5 & 1.5 & 1.5 & 1.5 \\
\hline & $>0.25-0.50$ & 3.0 & 2.5 & 2.5 & 2.5 & 2.0 & 2.0 & 2.0 & 2.0 & 2.0 \\
\hline & $>0.50-0.75$ & 3.5 & 3.0 & 3.0 & 3.0 & 2.5 & 2.5 & 2.5 & 2.5 & 2.5 \\
\hline & $>0.75-1.00$ & 4.0 & 3.5 & 3.5 & 3.5 & 3.0 & 3.0 & 3.0 & 3.0 & 3.0 \\
\hline & $>1.00$ & 5.0 & 4.0 & 4.0 & 4.0 & 3.5 & 3.5 & 3.0 & 3.0 & 3.0 \\
\hline \multirow{6}{*}{$>5-6$} & $0.00-0.25$ & 2.0 & 2.0 & 1.5 & 1.5 & 1.5 & 1.5 & 1.5 & 1.5 & 1.5 \\
\hline & $>0.25-0.30$ & 4.0 & 3.0 & 2.5 & 2.5 & 2.0 & 2.0 & 2.0 & 2.0 & 2.0 \\
\hline & $>0.30-0.50$ & 4.5 & 4.0 & 3.5 & 3.0 & 2.5 & 2.5 & 2.5 & 2.5 & 2.5 \\
\hline & $>0.50-0.75$ & 5.0 & 4.5 & 4.0 & 3.5 & 3.0 & 3.0 & 3.0 & 3.0 & 3.0 \\
\hline & $>0.75-1.00$ & 5.5 & 5.0 & 4.5 & 4.0 & 3.0 & 3.0 & 3.0 & 3.0 & 3.0 \\
\hline & $>1.00$ & 6.0 & 5.0 & 5.0 & 4.5 & 3.5 & 3.5 & 3.5 & 3.5 & 3.5 \\
\hline \multirow{6}{*}{$>6$} & $0.00-0.25$ & 4.0 & 3.0 & 2.5 & 2.5 & 2.5 & 2.5 & 2.0 & 2.0 & 1.0 \\
\hline & $>0.25-0.30$ & 4.5 & 4.0 & 3.5 & 3.5 & 3.5 & 3.0 & 2.5 & 2.5 & 2.5 \\
\hline & $>0.30-0.50$ & 5.0 & 4.5 & 4.0 & 4.0 & 3.5 & 3.0 & 2.5 & 2.5 & 2.5 \\
\hline & $>0.50-0.75$ & 5.5 & 5.0 & 4.5 & 4.5 & 4.0 & 3.5 & 3.0 & 3.0 & 3.0 \\
\hline & $>0.75-1.00$ & 6.0 & 5.5 & 5.0 & 5.0 & 4.5 & 4.0 & 3.5 & 3.5 & 3.5 \\
\hline & $>1.00$ & 7.0 & 6.0 & 5.5 & 5.5 & 5.0 & 4.5 & 4.0 & 4.0 & 4.0 \\
\hline
\end{tabular}

Note: Interpolation for percentage of trucks and buses is recommended to the nearest 0.1.

Fuente: HCM 2010 
Tabla 6: Ajuste de volumen de la demanda para vehículos RVs

\begin{tabular}{|c|c|c|c|c|c|c|c|c|c|c|}
\hline \multirow{2}{*}{$\begin{array}{c}\text { Upgrade } \\
(\%)\end{array}$} & \multirow{2}{*}{$\begin{array}{l}\text { Length } \\
\text { (mi) }\end{array}$} & \multicolumn{9}{|c|}{ Proportion of RVs } \\
\hline & & $2 \%$ & $4 \%$ & $5 \%$ & $6 \%$ & $8 \%$ & $10 \%$ & $15 \%$ & $20 \%$ & $\geq 25 \%$ \\
\hline$\leq 2$ & All & 1.2 & 1.2 & 1.2 & 1.2 & 1.2 & 1.2 & 1.2 & 1.2 & 1.2 \\
\hline \multirow{2}{*}{$>2-3$} & $0.00-0.50$ & 1.2 & 1.2 & 1.2 & 1.2 & 1.2 & 1.2 & 1.2 & 1.2 & 1.2 \\
\hline & $>0.50$ & 3.0 & 1.5 & 1.5 & 1.5 & 1.5 & 1.5 & 1.2 & 1.2 & 1.2 \\
\hline \multirow{3}{*}{$>3-4$} & $0.00-0.25$ & 1.2 & 1.2 & 1.2 & 1.2 & 1.2 & 1.2 & 1.2 & 1.2 & 1.2 \\
\hline & $>0.25-0.50$ & 2.5 & 2.5 & 2.0 & 2.0 & 2.0 & 2.0 & 1.5 & 1.5 & 1.5 \\
\hline & $>0.50$ & 3.0 & 2.5 & 2.5 & 2.5 & 2.0 & 2.0 & 2.0 & 1.5 & 1.5 \\
\hline \multirow{3}{*}{$>4-5$} & $0.00-0.25$ & 2.5 & 2.0 & 2.0 & 2.0 & 1.5 & 1.5 & 1.5 & 1.5 & 1.5 \\
\hline & $>0.25-0.50$ & 4.0 & 3.0 & 3.0 & 3.0 & 2.5 & 2.5 & 2.0 & 2.0 & 2.0 \\
\hline & $>0.50$ & 4.5 & 3.5 & 3.0 & 3.0 & 3.0 & 2.5 & 2.5 & 2.0 & 2.0 \\
\hline \multirow{3}{*}{$>5$} & $0.00-0.25$ & 4.0 & 3.0 & 2.5 & 2.5 & 2.5 & 2.0 & 2.0 & 2.0 & 1.5 \\
\hline & $>0.25-0.50$ & 6.0 & 4.0 & 4.0 & 3.5 & 3.0 & 3.0 & 2.5 & 2.5 & 2.0 \\
\hline & $>0.50$ & 6.0 & 4.5 & 4.0 & 4.0 & 3.5 & 3.0 & 3.0 & 2.5 & 2.0 \\
\hline
\end{tabular}

Note: Interpolation for percentage of RVs is recommended to the nearest 0.1 .

Fuente: HCM 2010

Los equivalentes para descensos específicos

El conocimiento de los impactos específicos de vehículos pesados en las condiciones de operación en rebaja es limitado. En general, si la rebaja no es suficiente a grave para cambiar a una marcha más baja (para participar de frenado del motor), los vehículos pesados pueden ser tratados como si estuvieran en segmentos de terreno llano. Cuando una rebaja es grave, los camiones a menudo deben utilizar marchas cortas para evitar aumentar demasiado la velocidad y correr fuera de control. En tales casos, su efecto sobre las condiciones de operación es más significativo que en terreno llano. En la Tabla 7 dan valores de ET para esta situación.

Tabla 7: Valores de los ET para diversas situaciones.

\begin{tabular}{c|c|cccc}
\hline $\begin{array}{c}\text { Downgrade } \\
(\%)\end{array}$ & $\begin{array}{c}\text { Length of } \\
\text { Grade (mi) }\end{array}$ & $\mathbf{5 \%}$ & \multicolumn{4}{|c}{ Proportion of Trucks and Buses } \\
\hline$<4$ & All & 1.5 & 1.5 & $15 \%$ & $\geq 20 \%$ \\
\hline $4-5$ & $\leq 4$ & 1.5 & 1.5 & 1.5 & 1.5 \\
& $>4$ & 2.0 & 2.0 & 2.0 & 1.5 \\
$>5-6$ & $\leq 4$ & 1.5 & 1.5 & 1.5 & 1.5 \\
& $>4$ & 5.5 & 4.0 & 4.0 & 3.5 \\
$>6$ & $\leq 4$ & 1.5 & 1.5 & 1.5 & 1.5 \\
& $>4$ & 7.5 & 6.0 & 5.5 & 4.5 \\
\hline
\end{tabular}

Fuente: HCM 2010 
Ajuste por población de conductores

Las características de la corriente de tráfico de base para los segmentos básicos de la autopista son representantes de los flujos de tráfico compuestos principalmente por los viajeros, o controladores que están familiarizados con la instalación de la autopista. El fp de ajuste se utiliza para reflejar el efecto de la población de conductores. Los valores de la gama fp son de 0,85 a 1,00 en la mayoría de los casos, aunque los valores más bajos tienden a observar casos aislados. En general, el analista debe utilizar un valor de 1,00, lo que refleja los pasajeros o conductores de otro tipo, acostumbrados, a menos que haya pruebas suficientes de que un valor más bajo se debe utilizar.

\section{¿Existe LOS F?}

En este punto, el volumen de la demanda se ha convertido en una tasa de flujo de demanda en vehículos de pasajeros por hora por carril en condiciones de bases equivalentes. Esta tasa de demanda debe compararse con la capacidad de la autopista de segmento básico.

$(2,400 \mathrm{veh} / \mathrm{h} / \mathrm{c}$ A partir de FFS $=121 \mathrm{~km} / \mathrm{h}$, y $113 \mathrm{~km} / \mathrm{h} ; 2,350 \mathrm{veh} / \mathrm{h} / \mathrm{c}$ partir de $\mathrm{FFS}=$ $105 \mathrm{~km} / \mathrm{h} ; 2300 \mathrm{veh} / \mathrm{h} / \mathrm{c}$ A partir de FFS = $97 \mathrm{~km} / \mathrm{h} ; 2,250 \mathrm{veh} / \mathrm{h} / \mathrm{c}$ A partir de FFS = 80 $\mathrm{km} / \mathrm{h})$.

Si la demanda es inferior o igual a la capacidad, el análisis continúa al Paso 5.

Paso 5: Estimación de velocidad y Capacidad

En este punto de la metodología, se han determinado lo siguiente: (a) la curva de la FFS y FFS apropiados para su uso en el análisis, y (b) la tasa de demanda expresada en vehículos de pasajeros por hora por carril debajo de las condiciones base de flujo equivalente. Con esta información, la velocidad estimada y la densidad del tráfico pueden ser determinadas.

Con las ecuaciones indicada a continuación, la velocidad esperada que significa flujo de tráfico puede ser calculado. Con esta velocidad estimada se determinará la densidad de la corriente de tráfico:

$$
\mathrm{D}=\frac{\mathrm{vp}}{\mathrm{s}}(\text { Ecu. } 8)
$$


Dónde:

$\mathrm{D}=$ densidad $(\mathrm{veh} / \mathrm{km} / \mathrm{c})$,

$v p=$ tasa de demanda de flujo (veh/h/c), y

$\mathrm{S}=$ velocidad media del flujo de tráfico en condiciones de base $(\mathrm{km} / \mathrm{h})$.

Como se ha señalado, esta ecuación se utiliza cuando el v/c es menor o igual a 1,00. Todos los casos en los que esta relación es mayor que 1,00 son niveles de servicio F. En estos casos, la velocidad S estará fuera de la gama de exposiciones.

Paso 6: Determinar Los Niveles de Servicio

En la tabla 8 se introduce con la densidad obtenida a partir de la ecuación anterior y se determina los niveles de servicios prevalecientes.

Tabla 4: Niveles de servicio con respecto a la densidad

\begin{tabular}{cc}
\hline LOS & Density $(\mathrm{pc} / \mathrm{mi} / \mathrm{ln})$ \\
\hline A & $\leq 11$ \\
B & $>11-18$ \\
C & $>18-26$ \\
D & $>26-35$ \\
E & $>35-45$ \\
F & Demand exceeds capacity \\
\end{tabular}

Fuente: HCM 2010 


\section{CAPITULO IV: CASO DE ESTUDIO}

\subsection{DESCRIPCIÓN DEL CASO DE ESTUDIO}

El caso de estudio se encuentra ubicado en el distrito de San Martín de Porres, provincia y departamento de Lima, en el área comprendida entre la intersección de la Panamericana Norte y el intercambio vial Tomas Valle en el Norte hasta la Panamericana Norte y Puente Control Zarumilla en el Sur.

La Panamericana Norte en el tramo que atraviesa el distrito de San Martín de Porras, es una vía de carácter Nacional, que se caracteriza por contar con fábricas, locales industriales, comerciales y viviendas, y forma parte de la red Vial Nacional, que atraviesa la ciudad cruzando otros distritos densamente poblados, por lo que su afluencia de tránsito es muy elevada; un volumen significativo de vehículos que transitan por ella corresponde a transporte de carga y transporte público.

La Av. Tomas Valle es una vía local que por el volumen de tráfico por el que se transita, puede ser considerada como troncal. Su capacidad física permite un alto nivel de movilidad e interconecta vías importantes como la Av. Túpac Amaru, Panamericana Norte Av. Universitaria, Atravesando en su recorrido todo el distrito de San Martín de Porres.

Tanto la Panamericana Norte como la Av. Tomas Valle cuentan con un gran aforo vehicular, que produce conflicto en su intersección, motivo por el cual el caso de estudio pretende plantear el inicio de reversibilidad de tránsito en la dirección del carril que se encuentra Sur -Norte.

Dentro del área de influencia de nuestro caso de estudio se encuentra el puente Control Zarumilla, este puente que está cerca del Intercambio vial Eduardo de Habich será el punto final en donde se pretende cerrar la reversibilidad de Carril en el tramo de SurNorte. 


\subsection{CLASIFICACIÓN DEL SEGMENTO DE AUTOPISTA EN CASO DE ESTUDIO}

En nuestro presente caso de estudio ubicado en la Panamericana Norte entre el intercambio vial de Tomas Valle y el puente control Zarumilla, existe 1 tipo de segmento de autopista (Ver Imagen 23), este tipo de autopista corresponden a:

Segmento de Autopista Básica.

La Clasificación de esta autopista es esencial, debido que de acuerdo al tipo de segmento se desarrollará la metodología específica para poder determinar los niveles de servicio. La sección cuenta con rampas de salida y de ingreso en ambos sentidos de la autopista.

Imagen 23: Caso de Estudio dividido en 3 Tramos

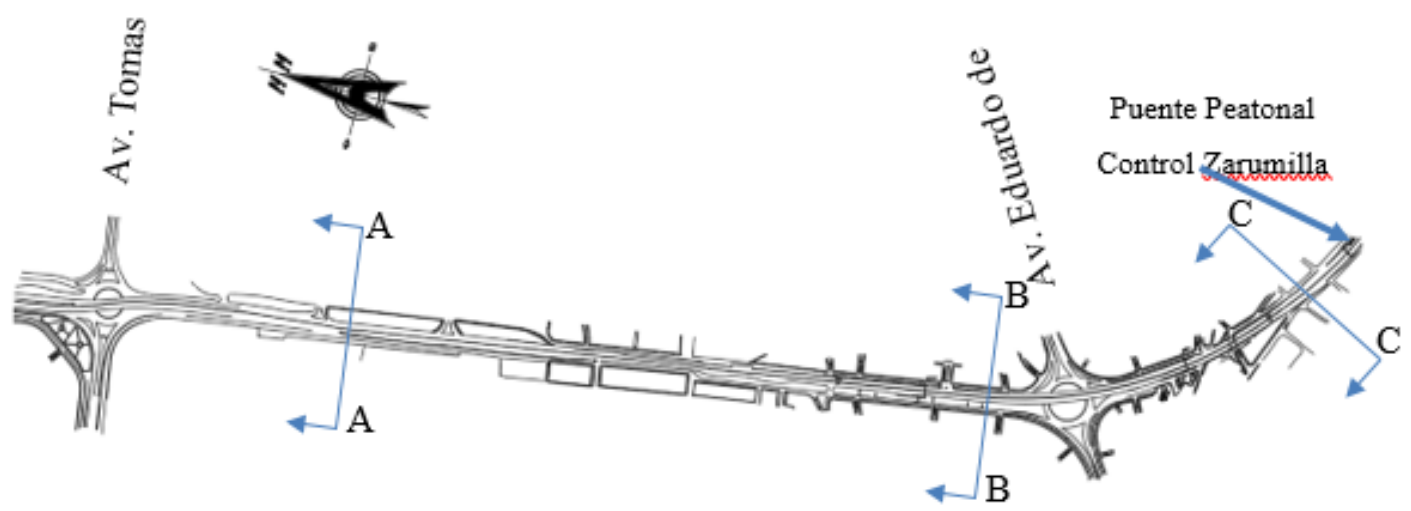

Fuente: Propia

A continuación, en la Imagen 24, 25, 26 y 27 se muestra las secciones transversales de la autopista de nuestro caso de estudio. Por lo general ambos sentidos comprenden 3 carriles de 3.5 m cada uno aproximadamente, además en la mayoría del trayecto cuenta con un aproximado de 40 centímetros de bordo libre en cada lado. En el Anexo 1 de este proyecto se encontrará a detalle estas secciones y la ubicación de este caso de estudio. 
Imagen 24: Segmento A - Intercambio Vial Tomas Valle

\section{PANAMERICANA NORTE SECCION A - A}

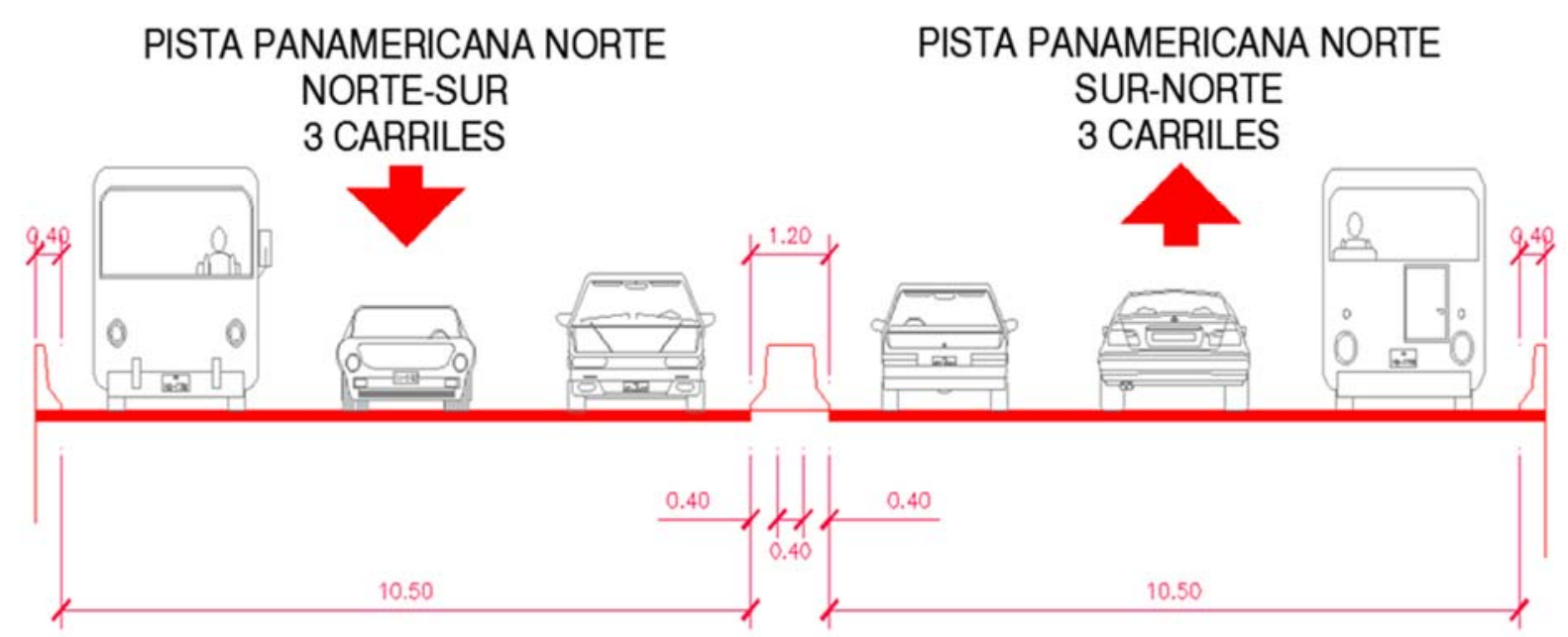

Fuente Propia

Imagen 25: Segmento B - Intercambio Vial Eduardo de Habich

PANAMERICANA NORTE

SECCION B - B

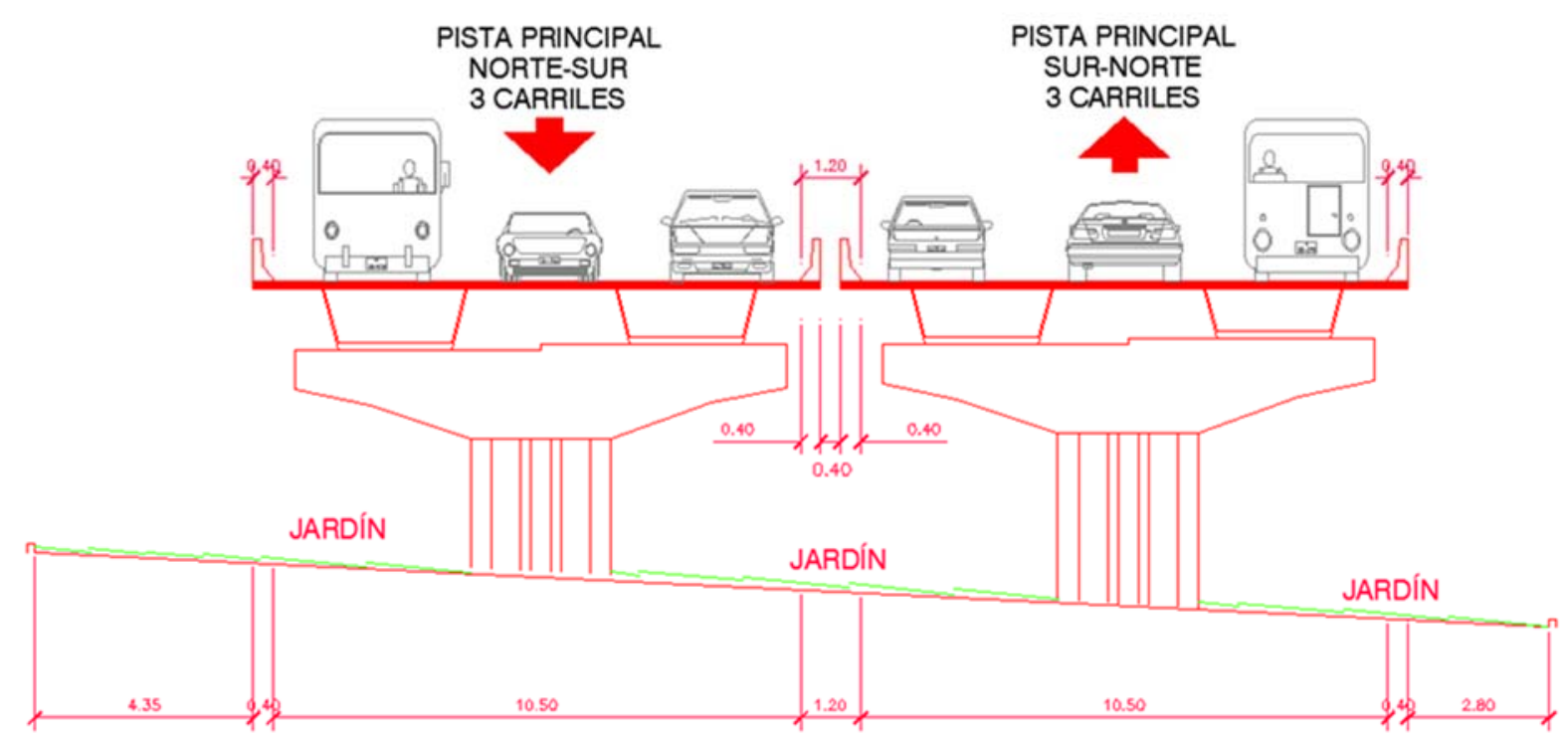

Fuente: Propia

Imagen 26: Sección C - Segmento de Autopista Básica 


\section{PANAMERICANA NORTE SECCION C - C}

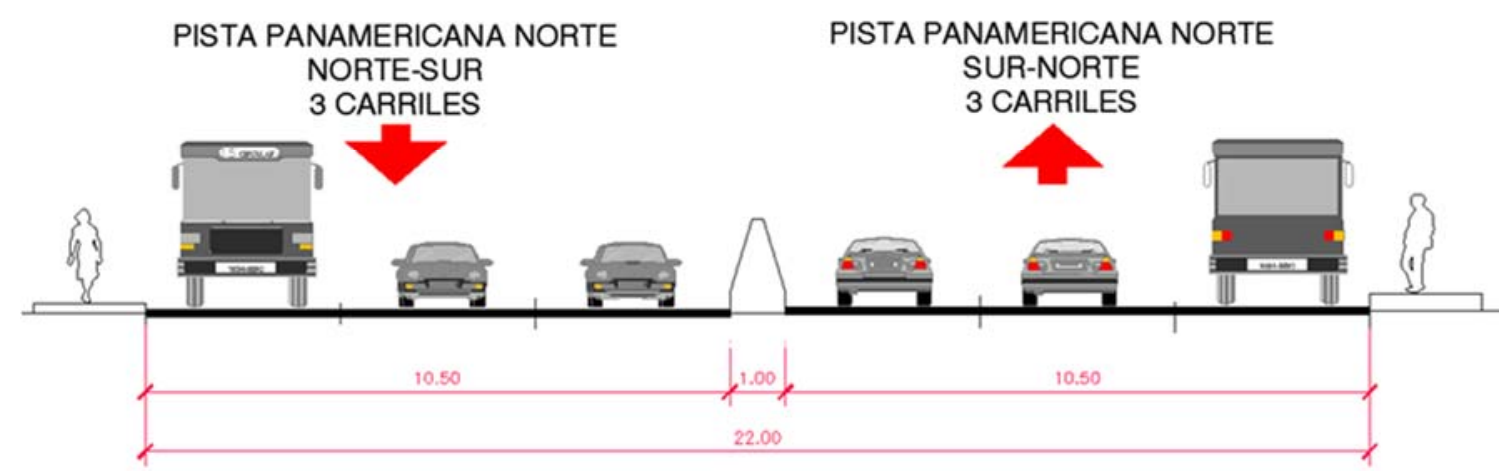

(Fuente: Propia)

Imagen 27: Vista en Planta del caso de estudio

\section{PANAMERICANA NORTE VISTA EN PLANTA}

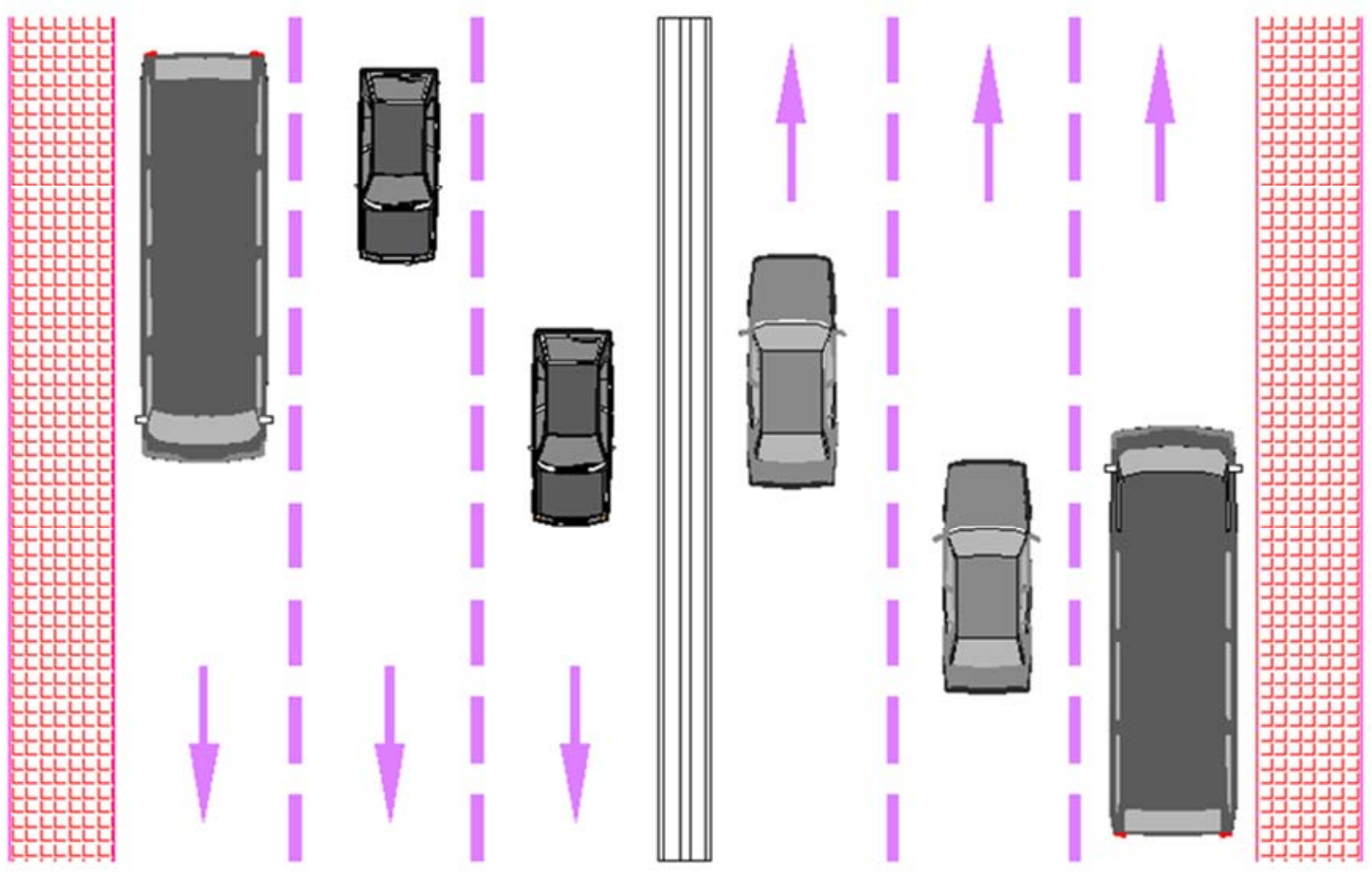

Fuente: Propia

\subsection{AFORO VEHICULARES DEL CASO DE ESTUDIO}

Para la toma de datos, se realizó un aforo con el formato adecuado (ver imagen 29) en las estaciones ubicadas en los puntos que se delimitan en el mapa (Imagen 28), esto permitirá 
obtener una completa información de campo, así como, la variabilidad del comportamiento vehicular.

Imagen 28: Puntos de aforos vehiculares (Fuente: Red Vial del distrito de SMP)

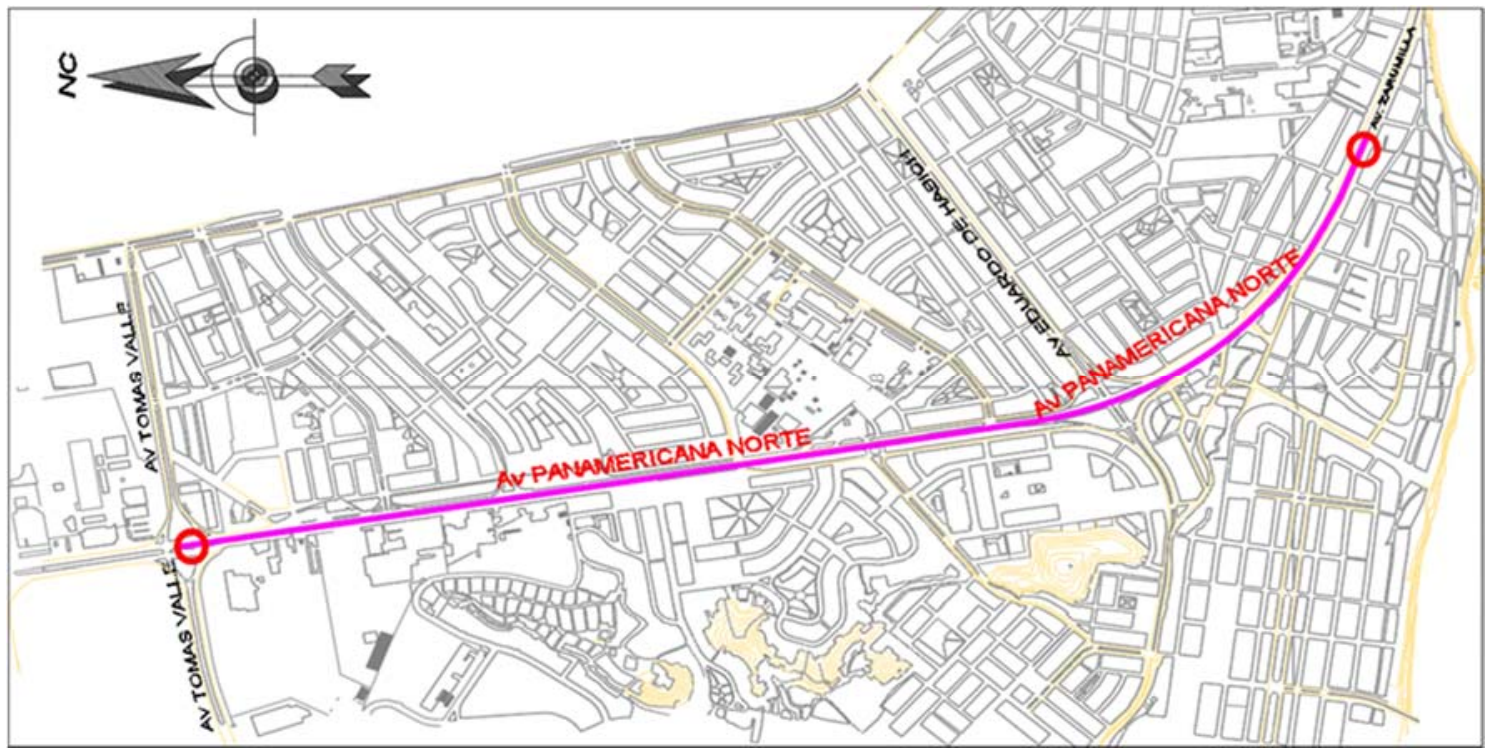

Fuente: Red Vial del distrito de SMP

Imagen 29: Plantilla de aforo vehicular

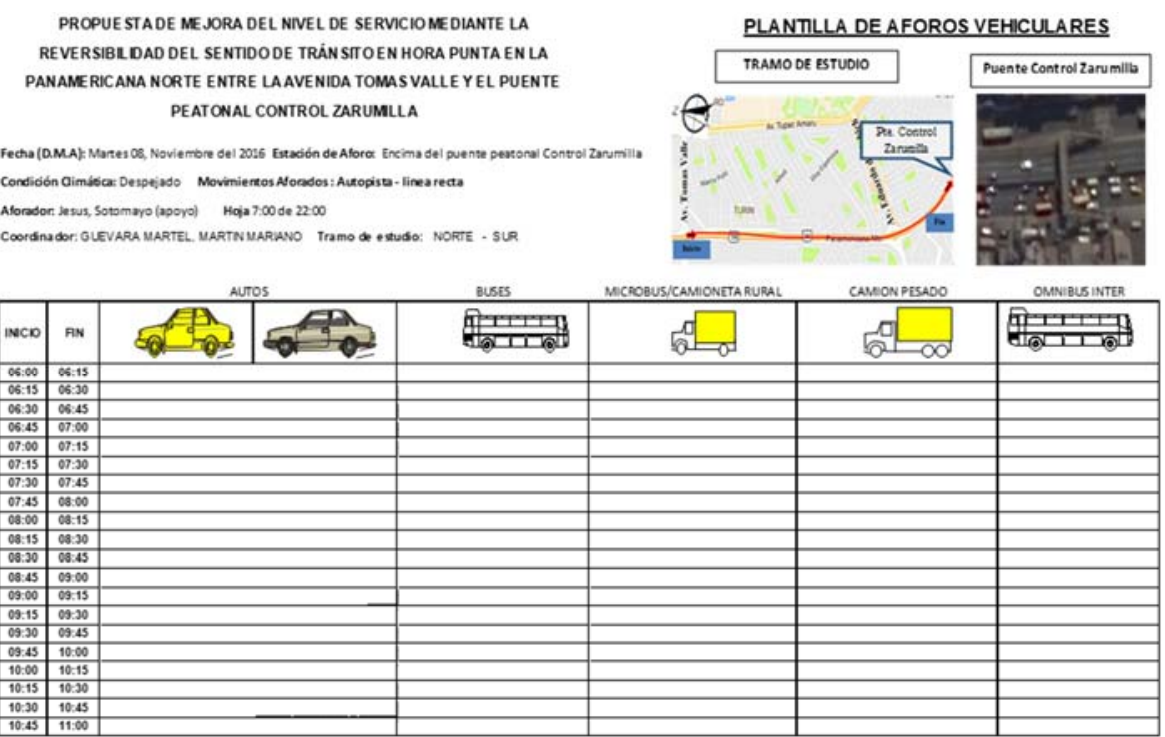

Fuente: MTC adecuado a nuestro caso de estudio 


\section{CAPITULO V: PLANTEAMIENTO DE SOLUCIÓN}

\subsection{DETERMINACIÓN DE HORARIOS}

Los días de aforo son designados según su representatividad, por ende, se optó aforar los días martes y jueves entre los meses de Noviembre del 2016 y Febrero del 2017, Mayo 2017 y Junio 2017, cuyos intervalos de hora fueron de 6:00 - 11:00 / 12:00 - 17:00 / 18:00 - 22:00 teniendo un total de 14 horas al día, cerrando la información cada cuarto de hora, para determinar la hora pico y el cuarto de hora pico. Por lo general, se trabaja con días representativos de la semana como martes, miércoles, jueves y sábado. Los días lunes, viernes y domingos suelen a poseer características atípicas.

En las tablas 11,12,13,14,15,16,17 y 18 mostramos el resumen de los aforos realizados en las estaciones de conteo vehicular donde especificamos el día de aforo, el horario de conteo cerrando cada 15 minutos, el trayecto de los vehículos y la cantidad de unidades vehiculares transformados a factores UCP. En el Anexo 2 del presente proyecto se encontrará todos los aforos vehiculares correspondientes a un ciclo anual.

Estación ubicada en el Puente Control Zarumilla. 
Tabla 9: Resumen del día martes del mes de noviembre del 2016.

Martes

HORAS

\begin{tabular}{|l|l|}
\hline NORTE - SUR & SUR - NORTE \\
\hline
\end{tabular} HORAS

NORTE - SUR SUR - NORTE

HORAS FACTOR UCP

NORTE - SUR SUR - NORTE

\begin{tabular}{|c|c|c|c|c|c|c|c|c|c|c|c|}
\hline & \multicolumn{2}{|c|}{ FACTOR UCP } & \multicolumn{4}{|c|}{ FACTOR UCP } & & & \multicolumn{2}{|c|}{ FACTOR UCP } \\
\hline 06:00 & 06:15 & 886 & 497 & $12: 00$ & 12:15 & 685 & 895 & 18:00 & 18:15 & 911 & 823 \\
\hline 06:15 & $06: 30$ & 1018 & 548 & $12: 15$ & $12: 30$ & 687 & 929 & 18:15 & 18:30 & 938 & 897 \\
\hline $06: 30$ & $06: 45$ & 1123 & 552 & $12: 30$ & $12: 45$ & 678 & 910 & $18: 30$ & $18: 45$ & 987 & 918 \\
\hline 06:45 & 07:00 & 1169 & 566 & $12: 45$ & $13: 00$ & 658 & 894 & 18:45 & 19:00 & 955 & 943 \\
\hline 07:00 & 07:15 & 1254 & 560 & $13: 00$ & $13: 15$ & 667 & 977 & 19:00 & 19:15 & 1169 & 975 \\
\hline 07:15 & 07:30 & 1341 & 567 & $13: 15$ & $13: 30$ & 731 & 912 & 19:15 & 19:30 & 1215 & 953 \\
\hline 07:30 & $07: 45$ & 1323 & 600 & $13: 30$ & $13: 45$ & 735 & 978 & 19:30 & 19:45 & 1424 & 968 \\
\hline 07:45 & 08:00 & 1416 & 624 & $13: 45$ & $14: 00$ & 713 & 954 & 19:45 & $20: 00$ & 1391 & 969 \\
\hline 08:00 & 08:15 & 1484 & 622 & $14: 00$ & $14: 15$ & 676 & 883 & $20: 00$ & $20: 15$ & 1228 & 974 \\
\hline 08:15 & 08:30 & 1625 & 651 & $14: 15$ & $14: 30$ & 753 & 895 & $20: 15$ & $20: 30$ & 1293 & 976 \\
\hline $08: 30$ & 08:45 & 1577 & 647 & $14: 30$ & $14: 45$ & 692 & 864 & $20: 30$ & $20: 45$ & 1129 & 934 \\
\hline $08: 45$ & 09:00 & 1544 & 650 & $14: 45$ & $15: 00$ & 649 & 967 & $20: 45$ & 21:00 & 1119 & 936 \\
\hline 09:00 & 09:15 & 1566 & 651 & $15: 00$ & $15: 15$ & 638 & 909 & 21:00 & 21:15 & 1076 & 1026 \\
\hline $09: 15$ & $09: 30$ & 1817 & 662 & $15: 15$ & $15: 30$ & 637 & 839 & 21:15 & $21: 30$ & 1082 & 990 \\
\hline 09:30 & 09:45 & 1744 & 689 & $15: 30$ & $15: 45$ & 677 & 846 & $21: 30$ & 21:45 & 1069 & 945 \\
\hline $09: 45$ & $10: 00$ & 1687 & 685 & $15: 45$ & $16: 00$ & 653 & 950 & $21: 45$ & $22: 00$ & 1083 & 979 \\
\hline $10: 00$ & $10: 15$ & 1633 & 667 & $16: 00$ & $16: 15$ & 676 & 884 & & & & \\
\hline $10: 15$ & $10: 30$ & 1584 & 689 & $16: 15$ & $16: 30$ & 644 & 830 & & & & \\
\hline $10: 30$ & $10: 45$ & 1583 & 695 & $16: 30$ & $16: 45$ & 709 & 891 & & & & \\
\hline $10: 45$ & 11:00 & 1518 & 675 & $16: 45$ & 17:00 & 709 & 877 & & & & \\
\hline
\end{tabular}

Tabla 10: Resumen del día jueves del mes de noviembre del 2016

JUEVES

HORAS NORTE-SUR SUR-NORTE HORAS NORTE-SUR SUR-NORTE HORAS NORTE-SUR SUR-NORTE

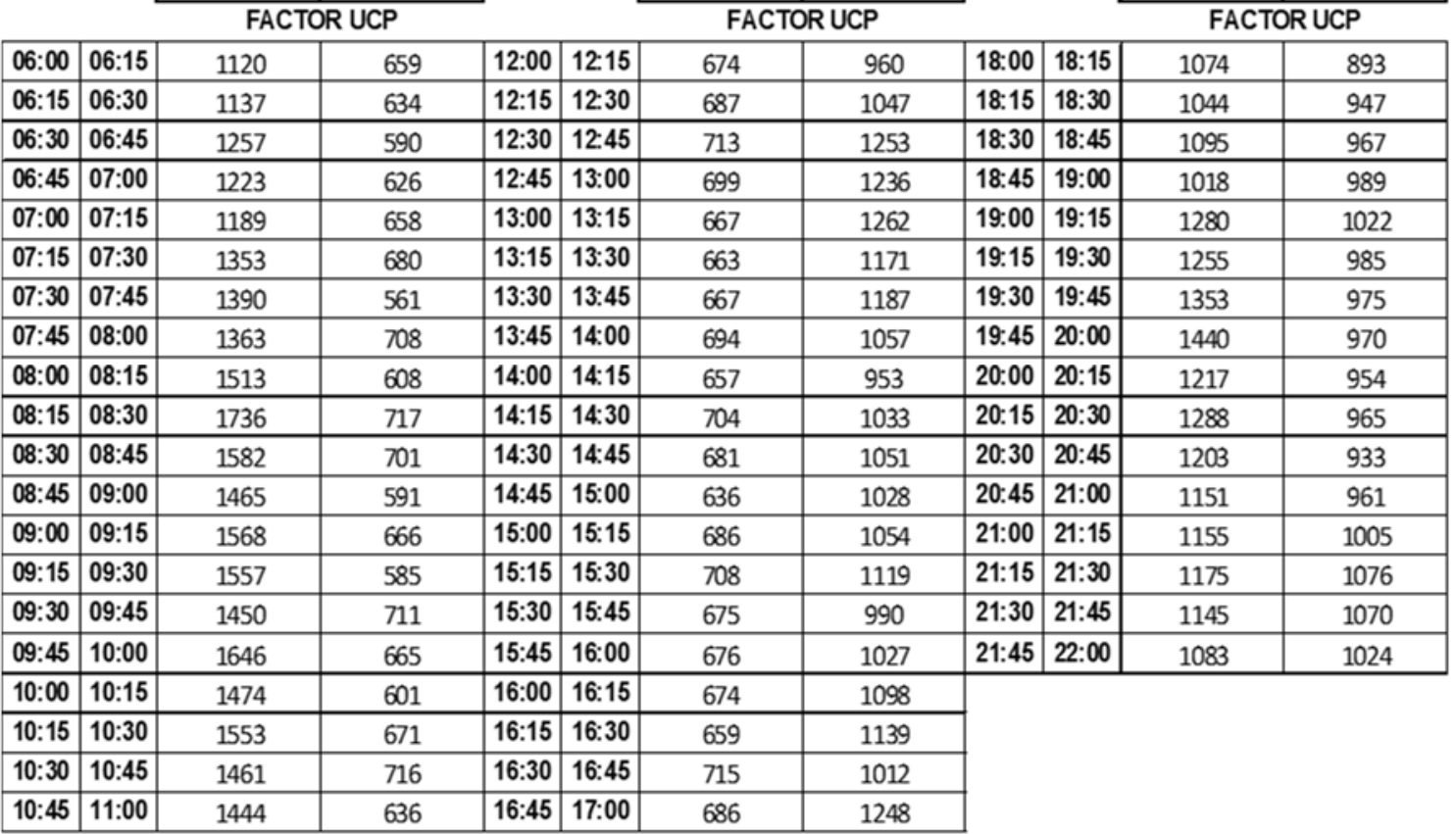


Tabla 5: Resumen del día martes del mes de febrero del 2017.

Martes

HORAS

\begin{tabular}{|l|l|}
\hline NORTE - SUR & SUR - NORTE \\
\hline
\end{tabular} HORAS

\begin{tabular}{|l|l|}
\hline NORTE - SUR & SUR - NORTE \\
\hline
\end{tabular} FACTOR UCP

HORAS

NORTE - SUR SUR - NORTE FACTOR UCP

\begin{tabular}{|c|c|c|c|c|c|c|c|c|c|c|c|}
\hline 06:00 & $06: 15$ & 1059 & 659 & $12: 00$ & $12: 15$ & 710 & 962 & $18: 00$ & 18:15 & 1337 & 990 \\
\hline $06: 15$ & $06: 30$ & 1843 & 571 & 12:15 & $12: 30$ & 699 & 985 & 18:15 & $18: 30$ & 1057 & 976 \\
\hline $06: 30$ & $06: 45$ & 1561 & 675 & $12: 30$ & $12: 45$ & 737 & 995 & $18: 30$ & \begin{tabular}{|l|}
$18: 45$ \\
\end{tabular} & 991 & 893 \\
\hline $06: 45$ & 07:00 & 1329 & 656 & $12: 45$ & $13: 00$ & 697 & 938 & $18: 45$ & 19:00 & 1202 & 906 \\
\hline 07:00 & 07:15 & 1292 & 628 & $13: 00$ & $13: 15$ & 681 & 931 & $19: 00$ & \begin{tabular}{|l}
$19: 15$ \\
\end{tabular} & 1302 & 887 \\
\hline 07:15 & 07:30 & 1728 & 654 & 13:15 & $13: 30$ & 710 & 900 & 19:15 & $19: 30$ & 1213 & 962 \\
\hline $07: 30$ & 07:45 & 1296 & 629 & $13: 30$ & $13: 45$ & 693 & 913 & $19: 30$ & 19:45 & 1119 & 900 \\
\hline $07: 45$ & 08:00 & 1251 & 573 & $13: 45$ & $14: 00$ & 740 & 935 & $19: 45$ & 20:00 & 1146 & 945 \\
\hline 08:00 & $08: 15$ & 1267 & 587 & $14: 00$ & 14:15 & 730 & 943 & $20: 00$ & $20: 15$ & 1048 & 966 \\
\hline 08:15 & $08: 30$ & 1636 & 592 & $14: 15$ & $14: 30$ & 746 & 918 & $20: 15$ & $20: 30$ & 1350 & 978 \\
\hline 08:30 & $08: 45$ & 1183 & 578 & $14: 30$ & 14:45 & 748 & 943 & $20: 30$ & $20: 45$ & 1025 & 927 \\
\hline 08:45 & $09: 00$ & 1416 & 677 & $14: 45$ & \begin{tabular}{|l|}
$15: 00$ \\
\end{tabular} & 696 & 903 & $20: 45$ & 21:00 & 1048 & 961 \\
\hline 09:00 & 09:15 & 1340 & 607 & $15: 00$ & $15: 15$ & 682 & 912 & 21:00 & 21:15 & 1155 & 989 \\
\hline $09: 15$ & $09: 30$ & 1422 & 603 & $15: 15$ & $15: 30$ & 742 & 916 & $21: 15$ & $21: 30$ & 1099 & 975 \\
\hline 09:30 & $09: 45$ & 1287 & 564 & $15: 30$ & $15: 45$ & 747 & 931 & $21: 30$ & $21: 45$ & 1384 & 935 \\
\hline $09: 45$ & $10: 00$ & 1429 & 565 & $15: 45$ & $16: 00$ & 709 & 960 & $21: 45$ & $22: 00$ & 1082 & 935 \\
\hline 10:00 & $10: 15$ & 1173 & 613 & 16:00 & $16: 15$ & 695 & 995 & & & & \\
\hline 10:15 & $10: 30$ & 1133 & 571 & $16: 15$ & $16: 30$ & 691 & 977 & & & & \\
\hline $10: 30$ & $10: 45$ & 1498 & 570 & $16: 30$ & $16: 45$ & 697 & 963 & & & & \\
\hline $10: 45$ & 11:00 & 1674 & 603 & $16: 45$ & $17: 00$ & 706 & 948 & & & & \\
\hline
\end{tabular}

FACTOR UCP

Tabla 12: Resumen del día jueves del mes de febrero del 2017

\section{JUEVES}

HORAS NORTE-SUR SUR - NORTE HORAS NORTE-SUR SUR-NORTE HORAS NORTE-SUR SUR-NORTE

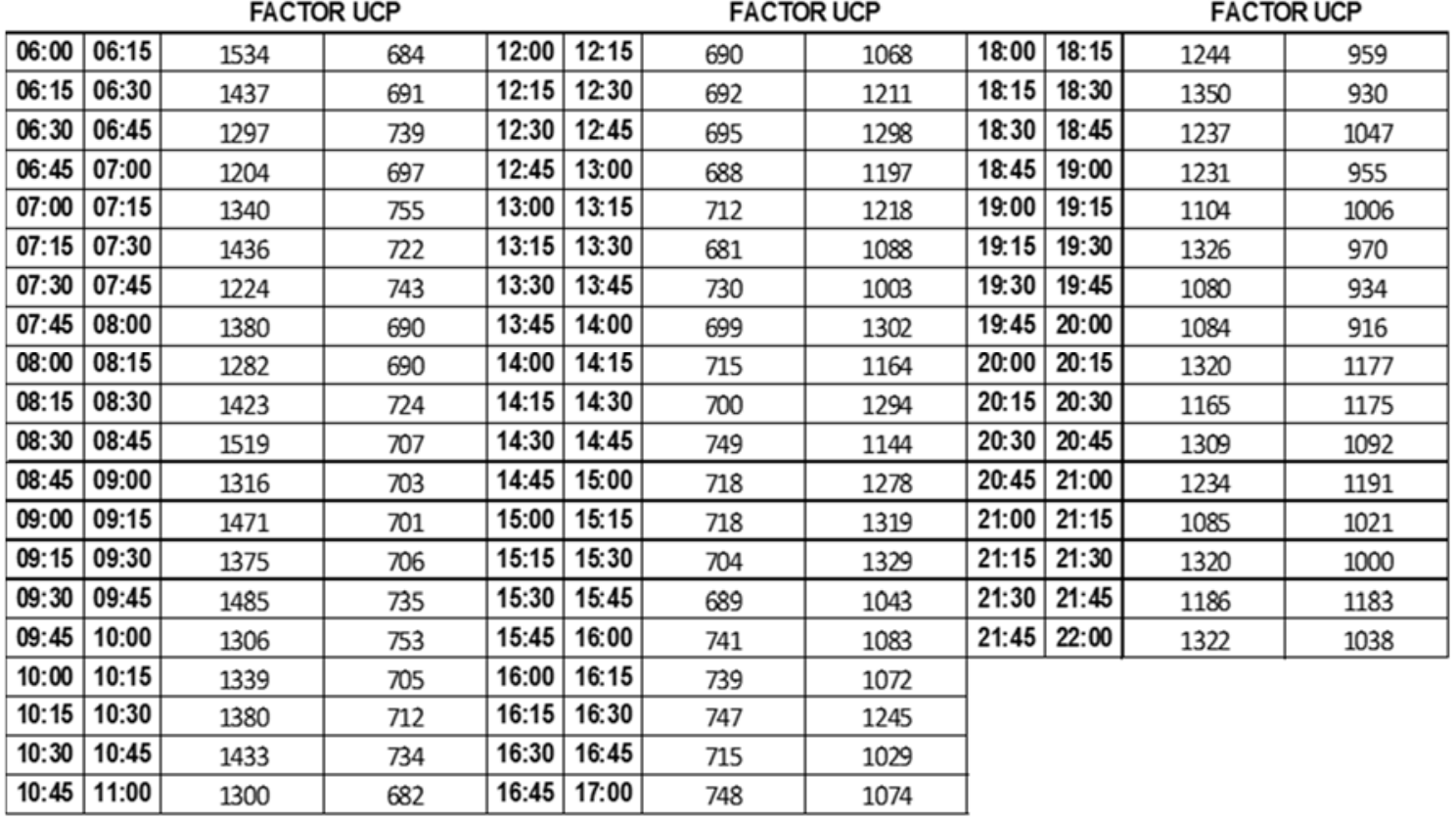


Estación ubicada en el intercambio vial Tomas Valle.

Tabla 13: Resumen del día martes del mes de noviembre del 2016.

\begin{tabular}{|c|c|c|c|c|c|c|c|c|c|c|c|}
\hline \multirow{2}{*}{\multicolumn{2}{|c|}{ HORAS }} & \multirow[b]{2}{*}{ NORTE - SUR } & \multirow{3}{*}{$\frac{\text { SUR - NORTE }}{\text { OR UCP }}$} & \multicolumn{4}{|c|}{ Martes } & \multirow{2}{*}{\multicolumn{2}{|c|}{ HORAS }} & \multirow[b]{2}{*}{ NORTE - SUR } & \multirow[b]{2}{*}{ SUR - NORTE } \\
\hline & & & & \multicolumn{2}{|c|}{ HORAS } & NORTE - SUR & SUR - NORTE & & & & \\
\hline & & FACTOR UCP & & \multicolumn{4}{|c|}{ FACTOR UCP } & & & \multicolumn{2}{|c|}{ FACTOR UCP } \\
\hline 06:00 & 06:15 & 1124 & 354 & 12:00 & 12:15 & 911 & \begin{tabular}{|l}
738 \\
\end{tabular} & 18:00 & 18:15 & 685 & \begin{tabular}{|r|}
894 \\
\end{tabular} \\
\hline 06:15 & $06: 30$ & 1110 & 388 & $12: 15$ & $12: 30$ & 938 & 805 & 18:15 & $18: 30$ & 687 & 903 \\
\hline 06:30 & 06:45 & 1227 & 394 & $12: 30$ & $12: 45$ & 987 & 830 & $18: 30$ & $18: 45$ & 678 & 900 \\
\hline 06:45 & 07:00 & 1271 & 394 & $12: 45$ & $13: 00$ & 955 & 849 & $18: 45$ & 19:00 & 658 & 887 \\
\hline 07:00 & 07:15 & 1376 & 398 & $13: 00$ & $13: 15$ & 1001 & 873 & 19:00 & 19:15 & 667 & 907 \\
\hline 07:15 & 07:30 & 1466 & 403 & 13:15 & $13: 30$ & 1077 & 849 & 19:15 & 19:30 & 731 & 914 \\
\hline 07:30 & 07:45 & 1447 & 424 & $13: 30$ & \begin{tabular}{|l|l|}
$13: 45$ \\
\end{tabular} & 1424 & 855 & 19:30 & $19: 45$ & 735 & 939 \\
\hline $07: 45$ & 08:00 & 1538 & 443 & $13: 45$ & $14: 00$ & 1391 & 855 & $19: 45$ & $20: 00$ & 713 & 924 \\
\hline 08:00 & 08:15 & 1604 & 441 & $14: 00$ & 14:15 & 1228 & 861 & $20: 00$ & $20: 15$ & 676 & 922 \\
\hline 08:15 & $08: 30$ & 1789 & 457 & 14:15 & $14: 30$ & 1293 & 863 & $20: 15$ & $20: 30$ & 753 & 935 \\
\hline $08: 30$ & $08: 45$ & 1710 & 458 & $14: 30$ & \begin{tabular}{|l|l}
$14: 45$ \\
\end{tabular} & 1129 & 831 & $20: 30$ & $20: 45$ & 692 & 904 \\
\hline 08:45 & 09:00 & 1668 & 463 & $14: 45$ & $15: 00$ & 1119 & 835 & $20: 45$ & 21:00 & 649 & 932 \\
\hline 09:00 & 09:15 & 1678 & 459 & $15: 00$ & $15: 15$ & 1076 & 905 & 21:00 & $21: 15$ & 638 & 928 \\
\hline 09:15 & $09: 30$ & 1941 & 471 & $15: 15$ & $15: 30$ & 1082 & 879 & 21:15 & $21: 30$ & 637 & 888 \\
\hline 09:30 & 09:45 & 1873 & 487 & $15: 30$ & \begin{tabular}{|l|l|}
$15: 45$ \\
\end{tabular} & 1069 & 845 & $21: 30$ & $21: 45$ & 677 & 914 \\
\hline $09: 45$ & $10: 00$ & 1803 & 484 & $15: 45$ & $16: 00$ & 1083 & 863 & 21:45 & 22:00 & 653 & 881 \\
\hline $10: 00$ & $10: 15$ & 1747 & 474 & $16: 00$ & $16: 15$ & 1112 & 874 & & & & \\
\hline $10: 15$ & $10: 30$ & 1694 & 489 & $16: 15$ & \begin{tabular}{|l}
$16: 30$ \\
\end{tabular} & 1124 & 871 & & & & \\
\hline $10: 30$ & $10: 45$ & 1695 & 492 & $16: 30$ & \begin{tabular}{|l|l|}
$16: 45$ \\
\end{tabular} & 1080 & 860 & & & & \\
\hline $10: 45$ & $11: 00$ & 1628 & 478 & $16: 45$ & $17: 00$ & 1097 & 864 & & & & \\
\hline
\end{tabular}

Tabla 14: Resumen del día jueves del mes de noviembre del 2016

JUEVES

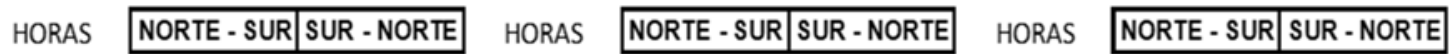
FACTOR UCP FACTOR UCP

\begin{tabular}{|c|c|c|c|c|c|c|c|c|c|c|c|}
\hline \multirow{2}{*}{\multicolumn{2}{|c|}{\begin{tabular}{|l|l|}
$06: 00$ & $06: 15$
\end{tabular}}} & & & \\
\hline & & 1134 & 366 & 12:00 & 12:15 & 1002 & 744 & 18:00 & 18:15 & 633 & 892 \\
\hline 06:15 & 06:30 & 1193 & 400 & 12:15 & $12: 30$ & 1157 & 825 & 18:15 & 18:30 & 795 & 873 \\
\hline $06: 30$ & $06: 45$ & 1186 & 427 & $12: 30$ & $12: 45$ & 1220 & 827 & $18: 30$ & 18:45 & 714 & 890 \\
\hline $06: 45$ & 07:00 & 1398 & 433 & 12:45 & 13:00 & 1049 & 827 & 18:45 & 19:00 & 697 & 897 \\
\hline 07:00 & 07:15 & 1407 & 423 & 13:00 & 13:15 & 1160 & 869 & 19:00 & 19:15 & 656 & 954 \\
\hline 07:15 & 07:30 & 1602 & 413 & 13:15 & $13: 30$ & 1103 & 816 & 19:15 & 19:30 & 718 & 935 \\
\hline $07: 30$ & 07:45 & 1613 & 455 & 13:30 & 13:45 & 1459 & 832 & 19:30 & 19:45 & 745 & 920 \\
\hline $07: 45$ & 08:00 & 1520 & 424 & $13: 45$ & $14: 00$ & 1396 & 841 & 19:45 & 20:00 & 734 & 918 \\
\hline 08:00 & 08:15 & 1676 & 423 & 14:00 & 14:15 & 1182 & 838 & 20:00 & 20:15 & 703 & 883 \\
\hline 08:15 & $08: 30$ & 1670 & 454 & 14:15 & $14: 30$ & 1186 & 874 & 20:15 & $20: 30$ & 724 & 941 \\
\hline $08: 30$ & $08: 45$ & 1754 & 459 & $14: 30$ & $14: 45$ & 1246 & 855 & $20: 30$ & $20: 45$ & 709 & 909 \\
\hline $08: 45$ & 09:00 & 1633 & 462 & 14:45 & 15:00 & 1250 & 828 & $20: 45$ & 21:00 & 699 & 893 \\
\hline 09:00 & 09:15 & 1758 & 439 & 15:00 & 15:15 & 1061 & 875 & 21:00 & 21:15 & 776 & 948 \\
\hline 09:15 & 09:30 & 1710 & 449 & $15: 15$ & $15: 30$ & 1227 & 859 & 21:15 & $21: 30$ & 662 & 907 \\
\hline $09: 30$ & $09: 45$ & 1802 & 474 & $15: 30$ & $15: 45$ & 1152 & 866 & $21: 30$ & 21:45 & 693 & 889 \\
\hline $09: 45$ & $10: 00$ & 1609 & 469 & $15: 45$ & $16: 00$ & 1012 & 871 & 21:45 & $22: 00$ & 686 & 927 \\
\hline 10:00 & $10: 15$ & 1615 & 469 & $16: 00$ & $16: 15$ & 1178 & 874 & & & & \\
\hline $10: 15$ & $10: 30$ & 1547 & 447 & $16: 15$ & $16: 30$ & 1044 & 895 & & & & \\
\hline $10: 30$ & $10: 45$ & 1512 & 425 & $16: 30$ & $16: 45$ & 1077 & 904 & & & & \\
\hline $10: 45$ & 11:00 & 1429 & 469 & $16: 45$ & $17: 00$ & 1110 & 863 & & & & \\
\hline
\end{tabular}


Tabla 15: Resumen del día martes del mes de noviembre del 2016

Martes

HORAS

NORTE - SUR SUR - NORTE FACTOR UCP HORAS

NORTE - SUR SUR - NORTE FACTOR UCP HORAS

NORTE - SUR SUR - NORTE FACTORUCP

\begin{tabular}{|c|c|c|c|c|c|c|c|c|c|c|c|}
\hline 06:00 & 06:15 & 1666 & 432 & \begin{tabular}{|l|l|}
$12: 00$ \\
\end{tabular} & 12:15 & 1029 & 863 & 18:00 & 18:15 & 761 & 886 \\
\hline 06:15 & 06:30 & 1688 & 465 & 12:15 & $12: 30$ & 1092 & 803 & $18: 15$ & $18: 30$ & 651 & 977 \\
\hline $06: 30$ & $06: 45$ & 1869 & 427 & $12: 30$ & $12: 45$ & 1091 & 914 & $18: 30$ & $18: 45$ & 715 & 867 \\
\hline 06:45 & 07:00 & 1803 & 468 & $12: 45$ & $13: 00$ & 1159 & 908 & 18:45 & 19:00 & 705 & 917 \\
\hline 07:00 & $07: 15$ & 1830 & 426 & 13:00 & 13:15 & 1154 & 939 & 19:00 & 19:15 & 761 & 905 \\
\hline 07:15 & 07:30 & 1305 & 425 & 13:15 & $13: 30$ & 1086 & 750 & 19:15 & $19: 30$ & 703 & 872 \\
\hline 07:30 & $07: 45$ & 1345 & 439 & $13: 30$ & $13: 45$ & 1043 & 765 & $19: 30$ & $19: 45$ & 740 & 989 \\
\hline 07:45 & 08:00 & 1510 & 486 & $13: 45$ & $14: 00$ & 1085 & 808 & 19:45 & 20:00 & 694 & 921 \\
\hline 08:00 & $08: 15$ & 1644 & 424 & 14:00 & $14: 15$ & 996 & 927 & $20: 00$ & $20: 15$ & 694 & 967 \\
\hline 08:15 & $08: 30$ & 1500 & 409 & 14:15 & $14: 30$ & 1282 & 866 & $20: 15$ & $20: 30$ & 689 & 899 \\
\hline 08:30 & $08: 45$ & 1692 & 466 & 14:30 & $14: 45$ & 1193 & 938 & $20: 30$ & $20: 45$ & 785 & 864 \\
\hline $08: 45$ & 09:00 & 1450 & 404 & 14:45 & $15: 00$ & 1053 & 847 & $20: 45$ & 21:00 & 654 & 995 \\
\hline 09:00 & $09: 15$ & 1302 & 427 & $15: 00$ & $15: 15$ & 1140 & 841 & 21:00 & 21:15 & 755 & 908 \\
\hline 09:15 & $09: 30$ & 1456 & 412 & $15: 15$ & $15: 30$ & 1042 & 786 & 21:15 & $21: 30$ & 698 & 992 \\
\hline 09:30 & $09: 45$ & 1683 & 452 & $15: 30$ & $15: 45$ & 1252 & 910 & 21:30 & 21:45 & 661 & 907 \\
\hline 09:45 & $10: 00$ & 1354 & 494 & $15: 45$ & $16: 00$ & 1209 & 794 & 21:45 & $22: 00$ & 693 & 942 \\
\hline $10: 00$ & $10: 15$ & 1582 & 400 & 16:00 & $16: 15$ & 1110 & 887 & & & & \\
\hline $10: 15$ & $10: 30$ & 1779 & 430 & 16:15 & $16: 30$ & 1110 & 833 & & & & \\
\hline $10: 30$ & $10: 45$ & 1587 & 462 & $16: 30$ & $16: 45$ & 1019 & 862 & & & & \\
\hline $10: 45$ & 11:00 & 1841 & 496 & $16: 45$ & $17: 00$ & 1276 & 825 & & & & \\
\hline
\end{tabular}

Tabla 16: Resumen del día jueves del mes de noviembre del 2016

\section{JUEVES}

HORAS NORTE-SUR SUR-NORTE HORAS NORTE-SUR SUR-NORTE HORAS NORTE-SUR SUR-NORTE

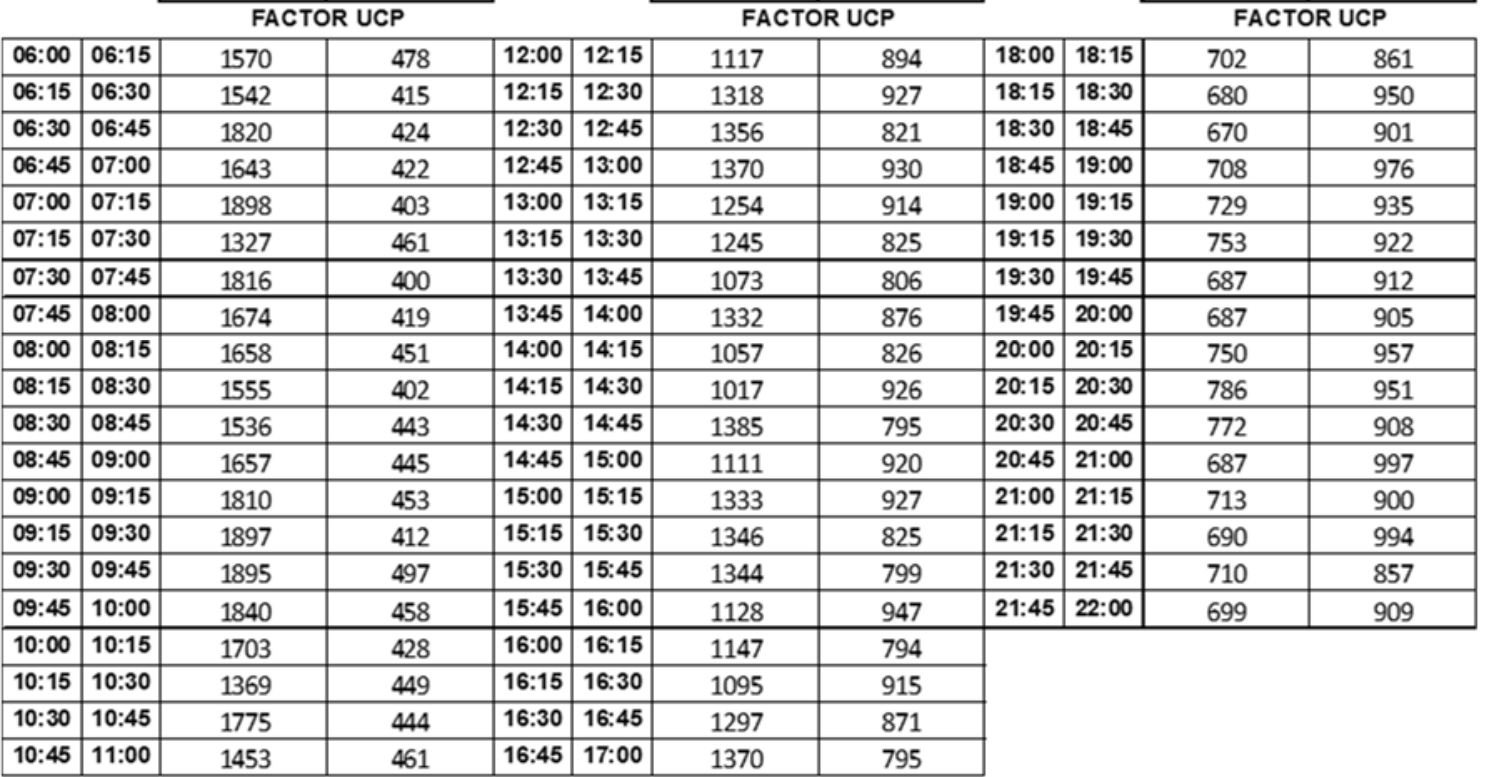




\subsection{PROTOCOLO DE IMPLEMENTACIÓN DE LA GESTIÓN DEL CARRIL REVERSIBLE}

Los dispositivos para la regulación del tránsito, deberán ubicarse con anterioridad al inicio del proyecto del carril reversible, permanecerán durante la ejecución de la misma y serán retirados una vez cesen las condiciones que dieron origen a su instalación.

Las señales deberán colocarse conforme al diseño, alineación de la vía y respetando el alineamiento geométrico, estos deben instalarse en ambos sentidos de la vía de tal forma que el conductor tenga suficiente tiempo para captar el mensaje, reaccionar y acatarlo.

La ampliación de un carril reversible se instalará al lado izquierda de la vía; en el tramo de Norte a Sur se incrementara un carril adicional, mientras que en el tramo de Sur a Norte se reducirá un carril, en el tramo del proyecto se instalaran señalizaciones pintadas en el pavimento y semaforizaciones áreas para optimizar la gestión de carriles reversibles, conjuntamente deberá haber señalizaciones áreas, señales preventivas y reglamentarias instaladas no menores a 100 metros tal como lo estipula el MANUAL DE DISPOSITIVOS DE CONTROL DEL TRANSITO AUTOMOTOR PARA CALLES Y CARRETERAS del MTC antes de comenzar el proyecto del carril reversible.

Estas señales automatizadas deben ir a la par con protocolos no autónomos como son los carros remolcadores, policías de tránsito, serenazgo e inspectores municipales, los cuales darán fluidez a la vía específicamente en el tramo del proyecto del carril reversible.

\subsubsection{Señales visibles}

Semaforización aérea para regular el uso de carriles: Estos semáforos se usarán durante un periodo de plazo determinado en la cual cierto carril será para el movimiento en un sentido dentro de unas horas y para el sentido opuesto durante otras horas el cual desempeñará las siguientes funciones (Ver Imagen 31); regular la velocidad de los vehículos para mantener la circulación continua a una velocidad constante, controlar la circulación por carriles y proporcionar un ordenamiento del tránsito. Tal como se estipula en el MTC en su "MANUAL DE DISPOSITIVOS DE CONTROL DEL TRANSITO AUTOMOTOR PARA CALLES Y CARRETERAS” en el inciso 6.7.1.3.2. 
Imagen 30: Semaforización aérea delimitando en cada carril

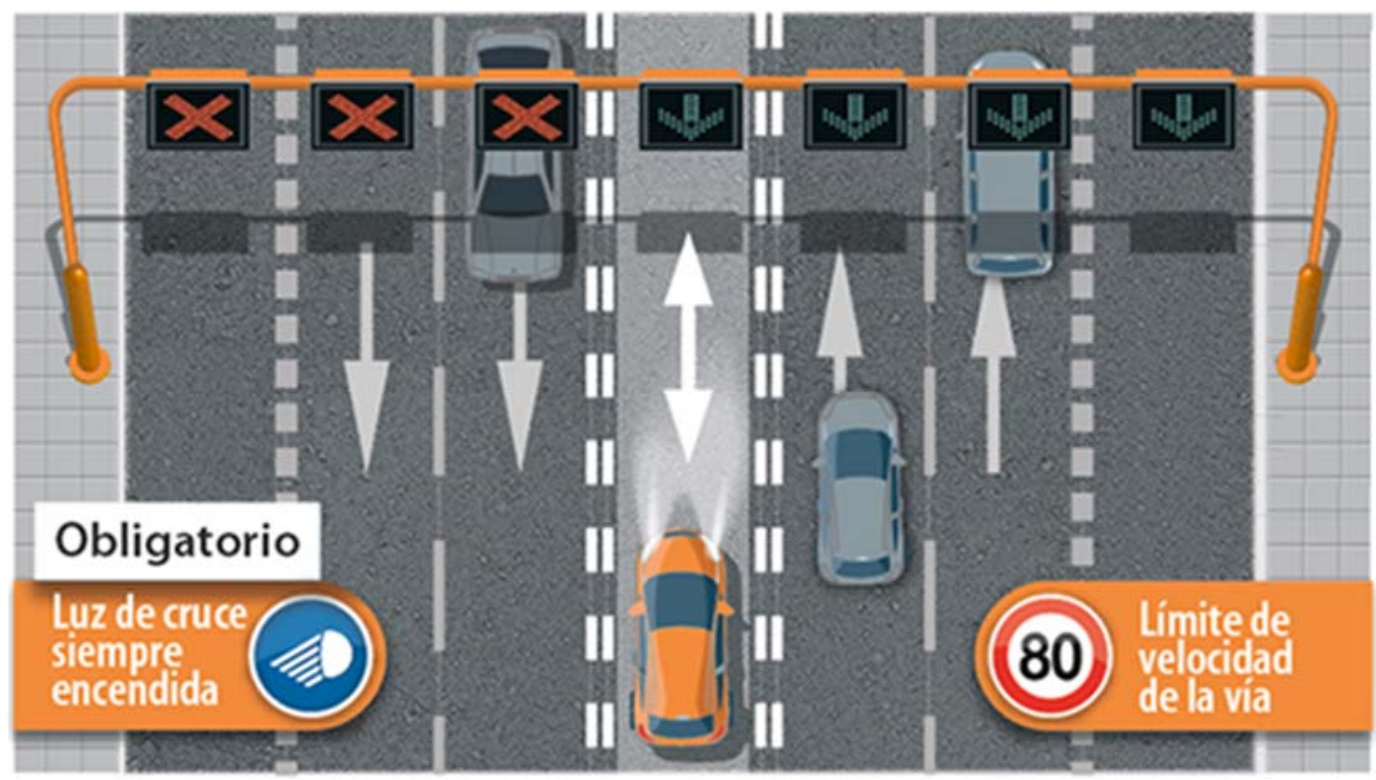

Imagen 31: Leyenda de las señalizaciones

\section{Semáforos de carril}
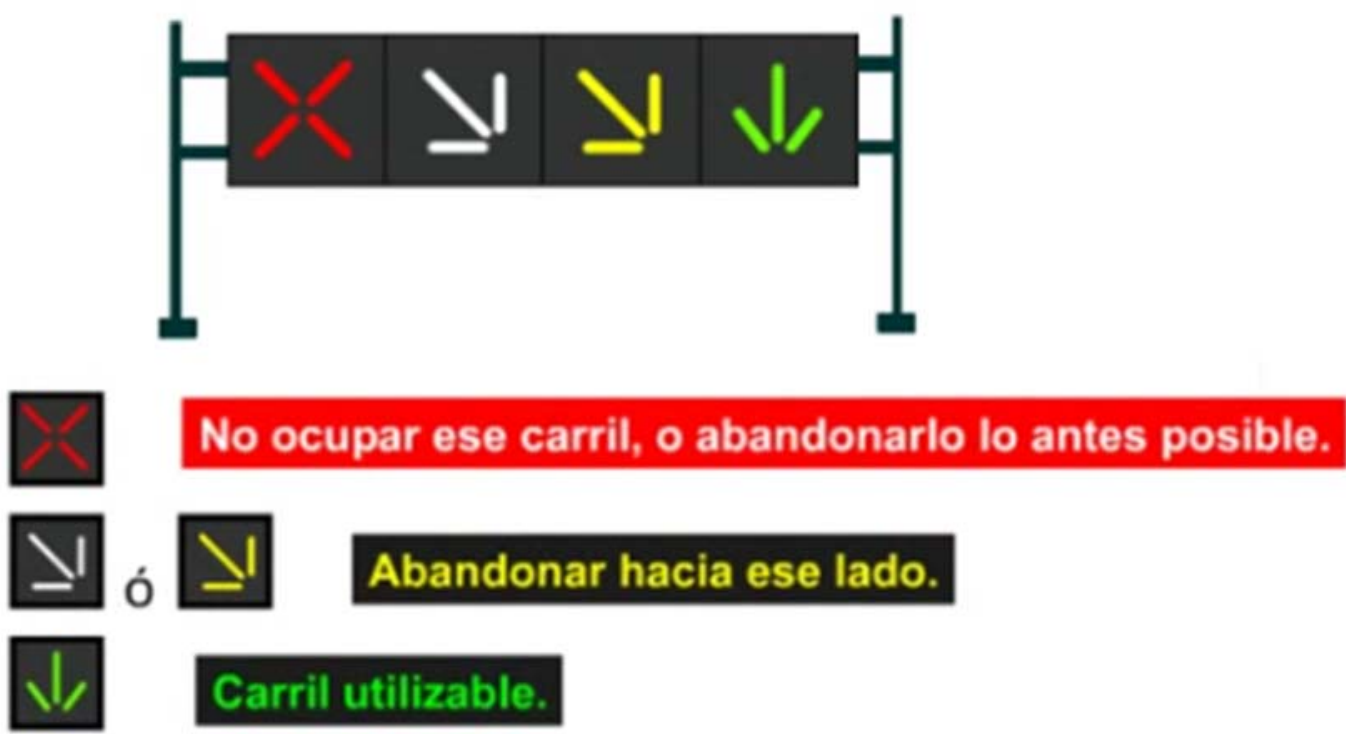

Fuente: Manual de dispositivos de control del tránsito automotor para calles y carreteras

Marcas en el pavimento: Estas tienen por finalidad complementar los dispositivos de control del tránsito, tales como las señales verticales, semáforos y otros, puesto que tiene la función de transmitir instrucciones y mensajes que otro tipo de dispositivo no lo puede 
hacer de forma efectiva. Estas marcas como se muestran en la Imagen 32, delimitarán el carril que se utilizara el cual de una mejora en la orientación del conductor.

Imagen 32: Marca en el pavimento en la entrada y salida de carril reversible
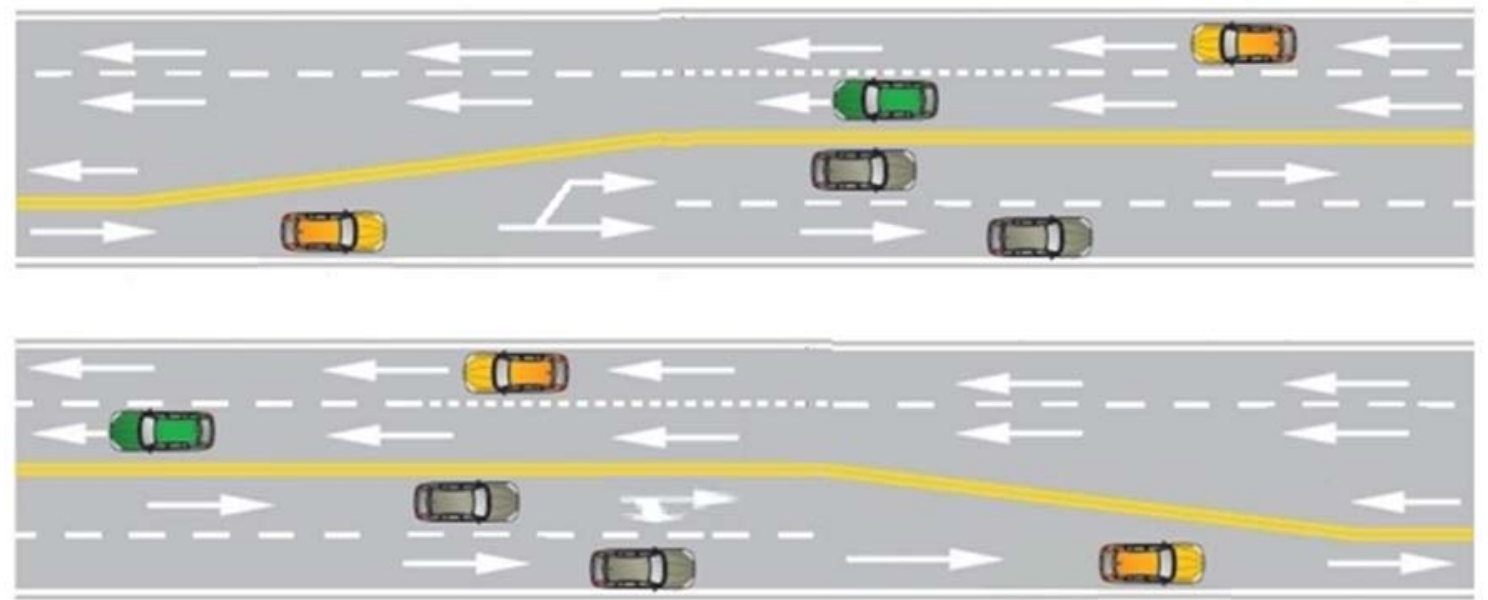

Fuente: Propia

Señalizaciones y controles de velocidad: La señalización vertical, así como los demás dispositivos de control de velocidad son necesarios para una la fluidez en del carril reversible y el carril que se encuentra aledaños a esta, de acuerdo al estudio de ingeniería vial que tome en consideración las condiciones particulares de cada carril tales como las referidas a velocidades máximas, reductores de velocidad y otros (Ver Imagen 33)

Imagen 33: Referencia de señales preventivas

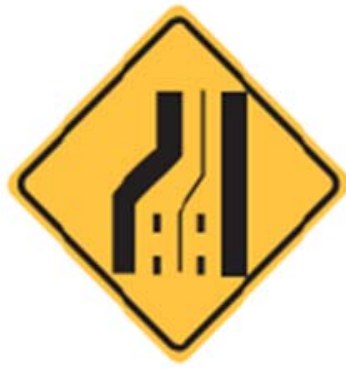

er.q

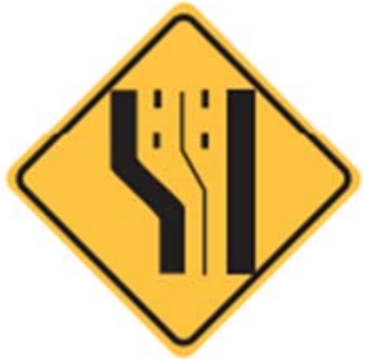

P-23

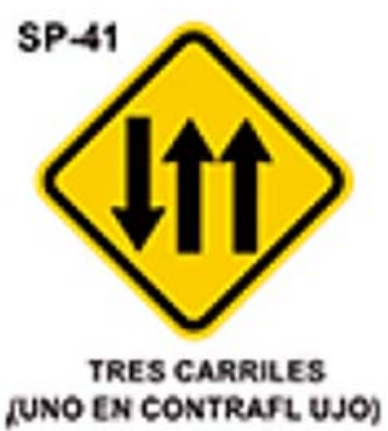

[UNO EN CONTRAFL UJO)

Fuente: manual de dispositivos de control del tránsito automotor para calles y carreteras

Conos o delineadores simples: Tienen por finalidad delinear o canalizar carriles o vías temporales de circulación, tales como: conos o delineadores simples, son de color anaranjado y deben contar con bandas de material retro reflectante (Ver Imagen 34) 
Imagen 34: Delimitacion de conos en la vía reversible

\section{En sentido contrario al habitual}

\section{Son frecuentes en autopistas y autovías para dar}

fluidez circulatoria en los accesos a ciudades.

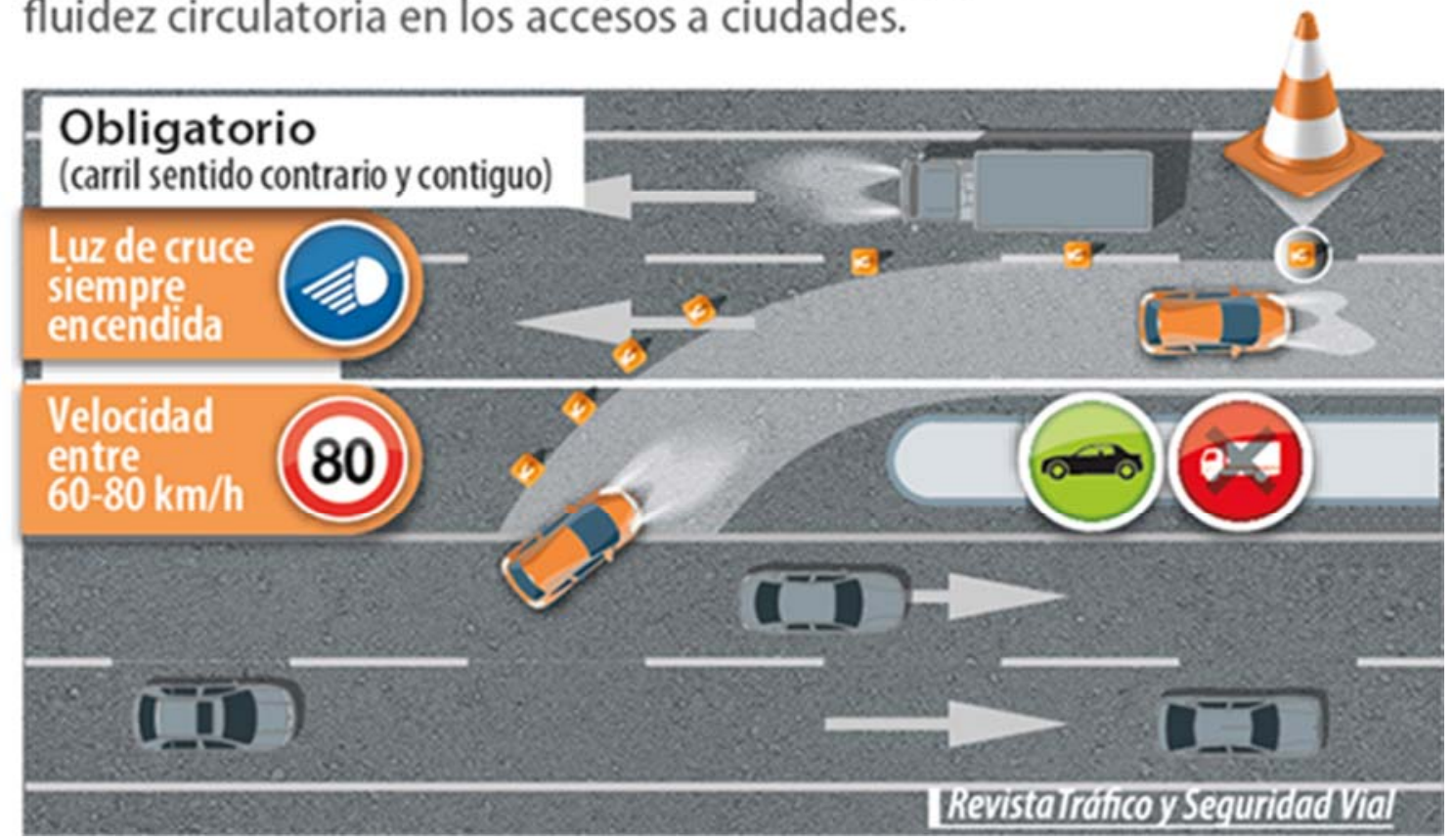

Fuente: Manual de dispositivos de control del tránsito automotor para calles y carreteras

Paneles publicitarios: Las señales que requieran una mayor permanencia en el sitio de las obras, se instalarán en soportes fijos y aquellas que requieran una menor permanencia, se instalarán en soportes portátiles como se muestra en la imagen 35.

La primera señal de prevención que advierta la existencia de la obra deberá colocarse aproximadamente a 400 metros antes de su inicio. Las vías alternas que faciliten el desvío de los vehículos del sitio de las obras, deben ser señalizadas convenientemente, de forma tal que permitan indicar tal situación. Para las arterias o vías de menor jerarquía, se recomienda colocar la primera señal a una distancia entre 100 y 200 metros. 


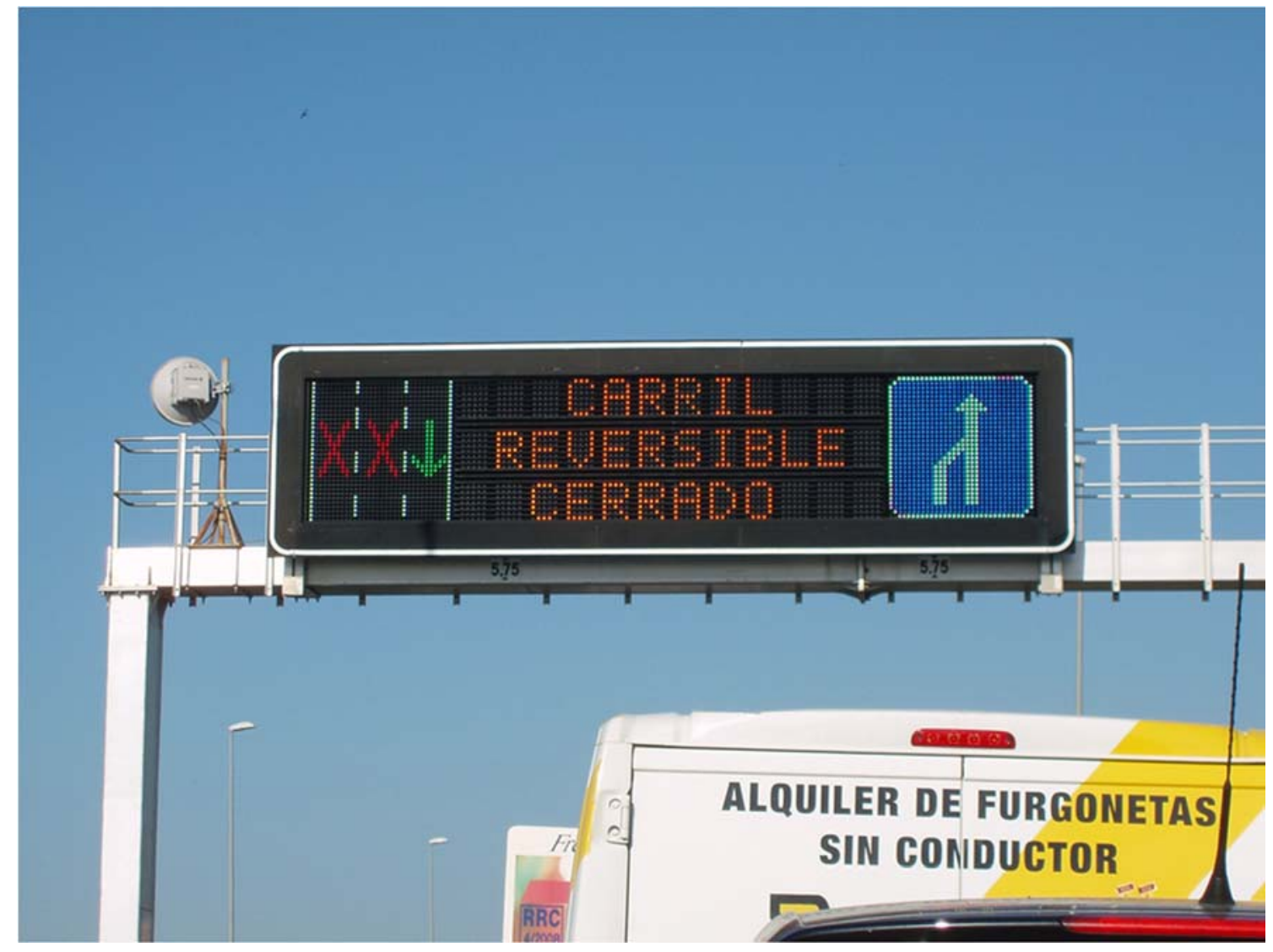

Fuente: Ayuntamiento de Madrid

\subsection{ANALISIS DE PROYECCIÓ FUTURA DEL TRANSITO.}

Según datos del Ministerio de Transportes y Comunicaciones, se observa que el departamento de Lima tiene un $80 \%$ de la totalidad de vehículos a nivel nacional y además de tener un crecimiento de $5 \%$ anual. Por este motivo, para que nuestra gestión de transito reversible en nuestro caso de estudio funcione adecuadamente a lo largo del tiempo, hicimos una proyección futura a 5 años contando desde el 2017. Para esto usamos la Tabla $\mathrm{N}^{\circ} 17$ donde se muestra los datos del parque automotor a detalle de la ciudad de Lima y Callao hasta 2015.

Haciendo una Proyección exponencial (Ver Imagen 36) obtenemos que para el 2022 tendremos en Lima 2,224,542.00 vehículos. Esto quiere decir que desde el año 2017 al 2022, el incremento del parque automotor será de un total de $27 \%$. 
Tabla 17: Parque Automotor Lima y Callao

\begin{tabular}{|c|c|c|c|c|c|c|c|c|c|c|}
\hline año & automovil & $\begin{array}{l}\text { stattion } \\
\text { wagon }\end{array}$ & $\begin{array}{l}\text { camioneta } \\
\text { pick up }\end{array}$ & $\begin{array}{c}\text { camioneta } \\
\text { panel }\end{array}$ & $\begin{array}{c}\text { camioneta } \\
\text { rural }\end{array}$ & Omnibus & camion & remolcador & total & \\
\hline 1993 & 259,616 & 36,159 & 78,422 & 6,712 & 39,274 & 21,619 & 48,254 & 4,522 & 494,578 & \\
\hline 2000 & 450,503 & 90,809 & 77,637 & 13,277 & 67,156 & 31,469 & 46,510 & 7,297 & 784,658 & \\
\hline 2001 & 473,479 & 95,440 & 81,597 & 13,954 & 70,581 & 33,073 & 48,882 & 7,670 & 824,676 & \\
\hline 2002 & 499,520 & 100,690 & 86,085 & 14,722 & 74,463 & 34,892 & 51,570 & 8,091 & 870,033 & \\
\hline 2003 & 529,491 & 106,731 & 91,250 & 15,605 & 78,931 & 36,986 & 54,664 & 8,577 & 922,235 & \\
\hline 2004 & 562,320 & 113,348 & 96,907 & 16,573 & 83,824 & 39,279 & 58,054 & 9,109 & 979,414 & \\
\hline 2005 & 579,189 & 116,749 & 99,815 & 17,070 & 86,339 & 40,458 & 59,795 & 9,382 & $1,008,797$ & \\
\hline 2006 & 599,461 & 120,835 & 103,308 & 17,66 & 89,361 & 41,874 & 61,888 & 9,710 & $1,044,104$ & \\
\hline 2007 & 623,439 & 125,668 & 107,440 & 18,374 & 92,935 & 43,548 & 64,364 & 10,099 & $1,085,867$ & \\
\hline 2008 & 651,494 & 131,323 & 112,275 & 19,201 & 97,118 & 45,508 & 67,260 & 10,553 & $1,134,732$ & \\
\hline 2009 & 680,811 & 137,233 & 117,328 & 20,065 & 101,488 & 47,556 & 70,287 & 11,028 & $1,185,796$ & \\
\hline 2010 & 711,448 & 143,408 & 122,607 & 20,968 & 106,055 & 49,696 & 73,449 & 11,524 & $1,239,155$ & \\
\hline 2011 & 743,463 & 149,862 & 128,125 & 21,911 & 110,827 & 51,932 & 76,755 & 12,043 & $1,294,918$ & \\
\hline 2012 & 776,919 & 156,606 & 133,890 & 22,897 & 115,814 & 54,269 & 80,209 & 12,585 & $1,353,189$ & \\
\hline 2013 & 811,880 & 163,653 & 139,915 & 23,928 & 121,026 & 56,711 & 83,818 & 13,151 & $1,414,082$ & \\
\hline 2014 & 848,415 & 171,017 & 146,212 & 25,004 & 126,472 & 59,263 & 87,590 & 13,743 & $1,477,716$ & \\
\hline 2015 & 886,593 & 178,713 & 152,791 & 26,130 & 132,164 & 61,930 & 91,531 & 14,361 & $1,544,213$ & \\
\hline 2016 & & & & & & & & & $1,663,878$ & \\
\hline 2017 & & & & & & & & & $1,746,391$ & \\
\hline 2018 & & & & & & & & & $1,832,995$ & \\
\hline 2019 & & & & & & & & & $1,923,894$ & \\
\hline 2020 & & & & & & & & & $2,019,300$ & \\
\hline 2021 & & & & & & & & & $2,119,438$ & \\
\hline 2022 & & & & & & & & & $2,224,542$ & \\
\hline
\end{tabular}

Fuente:

II_Plan_Integral_de_Saneamiento_Atmosferico_Lima_Callao_PISA_2011_2015

Imagen 36: Proyección del Parque Automotor

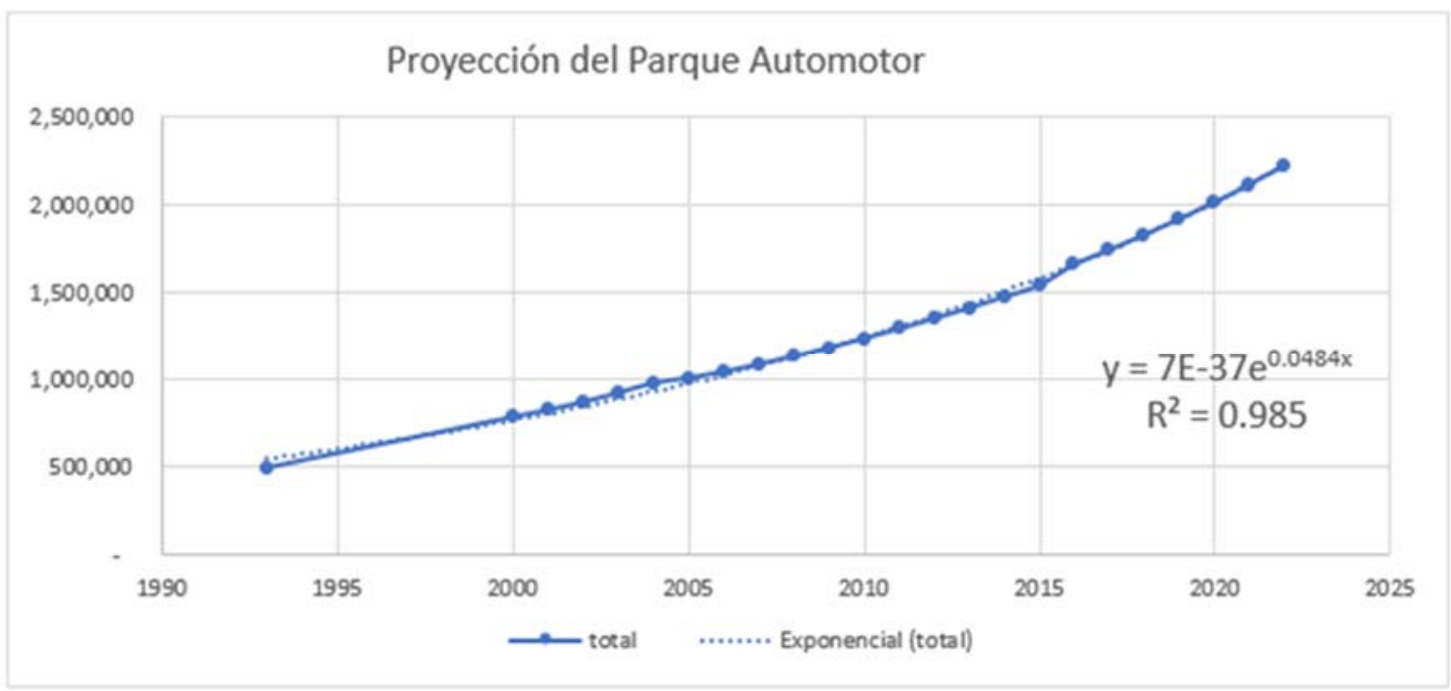

\subsection{PLANTEAMIENTO DE NIVELES DE SERVICIO}

En este capítulo se define los modelos propuestos para el cálculo de los niveles de servicio de nuestra sección de autopista básica del caso de estudio. Las propuestas de este 
planteamiento son basadas en los datos de tráfico obtenidos en nuestras estaciones de toma de datos realizados en los respectivos aforos.

\subsubsection{Niveles de servicio existente}

En nuestro caso de estudio tenemos como alcance 6 carriles divididos en dos direcciones, de las cuales según el aforo respectivo hallaremos la capacidad y los niveles de servicio existentes. Este modelo nos permitirá crear un patrón de referencia de los estados de nuestras vías utilizando la velocidad de flujo libre calculado en gabinete. Como primer planteamiento de los niveles de servicio tomaremos a nuestra estación Puente Control Zarumilla del aforo del día martes del mes de noviembre en dirección de Sur - Norte para hacer los cálculos detallados.

El análisis operacional para el presente trabajo tiene como volumen de demanda 2736.00 $\mathrm{veh} / \mathrm{h}$.

Paso 1: Datos de entrada

- Volumen de 2736.00 vehículos / h (una dirección, existente)

- Composición del tráfico: 5\% vehículos con más de 2 ejes

- Tres carriles en cada dirección

- FFS $=65 \mathrm{mi} / \mathrm{h}$ (medido)

- $\mathrm{PHF}=0,98$

- Tráfico de cercanías (usuarios regulares)

Paso 2: Calcular FFS

Usamos la ecuación 5 del capítulo de metodología de autopista de segmento básico:

$$
F F S=75.4-f l w-f l c-3.22 T R D^{0.84}
$$

Haciendo uso de las tablas y formula en el capítulo de metodología para el cálculo del FFS en segmento de autopista básica. Tenemos un FFS igual a $65 \mathrm{mi} / \mathrm{hr}$.

Paso 3: Seleccionar Curva FFS 
El paso 3 no es necesario. Se utilizará la curva FFS para $65 \mathrm{mi} / \mathrm{h}$, basada en la

Valor medido.

Paso 4: Ajustar el volumen de demanda

En este caso, se ajustarán el volumen de demanda actual usando la Ecuación 6:

$$
\mathrm{Up}=\frac{\mathrm{V}}{P H F \times N \times f H V \times \mathrm{fp}}
$$

El factor de hora pico (PHF) se da como 0,95, y hay tres carriles en cada dirección. El Factor de ajuste de la población de conductores para usuarios regulares será 1,00. El factor para vehículos pesados debe reflejar un 5\% de camiones en la vía. De la tabla N³ $\mathrm{PCE}$ para camiones en terreno nivelado es de 1.5, mientras que para vehículos menores a 2 ejes es de 1.2. La ecuación 7 da entonces lo siguiente:

$$
f H V=\frac{1}{1+P t(E t-1)+\operatorname{Pr}(E r-1)}
$$

\begin{tabular}{|l|r|}
\hline$f_{\mathrm{HV}}$ & 0.82 \\
\hline $\mathrm{P}_{\mathrm{T}}$ & $5 \%$ \\
\hline $\mathrm{P}_{\mathrm{R}}$ & $95 \%$ \\
\hline $\mathrm{E}_{\mathrm{T}}$ & 1.5 \\
\hline $\mathrm{E}_{\mathrm{R}}$ & 1.2 \\
\hline
\end{tabular}

Se calcularán dos valores de VP: uno para las condiciones presentes

$$
\mathrm{Up}(\text { presente })=\frac{2736}{0.98 \times 3 \times 0.823 \times 0.90}=1256 \mathrm{pc} / \mathrm{h}
$$

Paso 5: Estimar la velocidad y densidad

Se calcularon los valores de velocidad y densidad. La tabla 8 se utilizará para estimar velocidades. La misma ecuación se aplica a ambos casos, además de un mismo FFS con un flujo sobre los $1400 \mathrm{pc} / \mathrm{h} / \mathrm{km}$.

$$
\mathrm{S}(\text { Presente })=65-0.00001418(\mathrm{Vp}-1.400)^{2}
$$




$$
\mathrm{S}(\text { Presente })=64.7 \mathrm{mi} / \mathrm{hr}
$$

Luego pasamos a calcular la demanda correspondiente.

$$
\begin{gathered}
D=\frac{v p}{S} \\
D(\text { presente })=\frac{1256}{64.70}=19.4 \mathrm{pc} / \mathrm{mi} / \mathrm{ln}
\end{gathered}
$$

Paso 6: Determinar LOS

De la tabla 9, los niveles de servicio de la situación presente es $C$, ya que se encuentra en un rango de densidad de 18 a $26 \mathrm{veh} / \mathrm{mi} / \mathrm{c}$.

\subsubsection{Niveles de servicio planteando la solución}

Para la mejora de los niveles de servicio en nuestro caso de estudio se plantea el carril reversible lo cual tiene como solución prestar un carril a la dirección más congestionada (en este caso el tramo Norte - Sur), dejando dicho tramo con 4 carriles en esa dirección y el otro (Tramo Sur - Norte) con 2 carriles. Este modelo nos permitirá crear un patrón de referencia de cómo puede mejorar los niveles de servicio en el tramo más congestionado (Norte - Sur) además de verificar si dicho planteamiento altera la capacidad del tramo Sur - Norte. Además, se introduce un elemento de planificación que es el crecimiento del tráfico esperado por lo que se determinará otro volumen de demanda de $3475 \mathrm{veh} / \mathrm{h}$ esperado en 5 años.

Como primer planteamiento de los niveles de servicio tomaremos a nuestra estación Puente Control Zarumilla del aforo del día martes en dirección de Sur - Norte para hacer los cálculos detallados.

Paso 1: Datos de entrada

- Volumen de 2736.00 vehículos / h (una dirección, existente)

- Volumen de 3475 veh/ h (una dirección, en 5 años)

- Composición del tráfico: 5\% vehículos con más de 2 ejes

- 2 carriles en dirección Sur - Norte 
- FFS $=65 \mathrm{mi} / \mathrm{h}$ (medido)

- $\mathrm{PHF}=0,98$

- Crecimiento del tráfico después de 5 años $=27 \%$

Paso 2: Calcular FFS

Usamos la ecuación 5 del capítulo de metodología de autopista de segmento básico:

$$
F F S=75.4-f l w-f l c-3.22 T R D^{0.84}
$$

Haciendo uso de las tablas y formula en el capítulo de metodología para el cálculo del FFS en segmento de autopista básica. Tenemos un FFS igual a $56 \mathrm{mi} / \mathrm{hr}$.

Paso 3: Seleccionar Curva FFS

El paso 3 no es necesario. Se utilizará la curva FFS para $65 \mathrm{mi} / \mathrm{h}$, basada en la

Valor medido.

Paso 4: Ajustar el volumen de demanda

En este caso, se ajustarán dos volúmenes de demanda (Actual y Futura) usando la Ecuación 6:

$$
\mathrm{Up}=\frac{\mathrm{V}}{P H F \times N \times \mathrm{fHV} \times \mathrm{fp}}
$$

El factor de hora pico (PHF) se da como 0,98, y hay 2 carriles en dirección Sur - Norte. El Factor de ajuste de la población de conductores para usuarios regulares será 0,90. El factor para vehículos pesados debe reflejar un 5\% de camiones en la vía. De la tabla $\mathrm{N}^{\circ} 3$ PCE para camiones en terreno nivelado es de 1.5, mientras que para vehículos menores a 2 ejes es de 1.2. La ecuación 7 da entonces lo siguiente:

$$
f H V=\frac{1}{1+\operatorname{Pt}(E t-1)+\operatorname{Pr}(E r-1)}
$$

\begin{tabular}{|l|r|}
\hline$F_{\text {HV }}$ & 0.82 \\
\hline $\mathrm{P}_{\mathrm{T}}$ & $5 \%$ \\
\hline
\end{tabular}




\begin{tabular}{|l|r|}
\hline $\mathrm{P}_{\mathrm{R}}$ & $95 \%$ \\
\hline $\mathrm{Et}_{\mathrm{T}}$ & 1.5 \\
\hline $\mathrm{E}_{\mathrm{R}}$ & 1.2 \\
\hline
\end{tabular}

Se calcularán dos valores de vp: uno para las condiciones presentes y uno para

Condiciones en 5 años:

$$
\begin{gathered}
v p(\text { presente })=\frac{2736}{0.98 \times 2 \times 0.823 \times 0.90}=1886 \mathrm{veh} / \mathrm{h} \\
v p(\text { futuro })=\frac{3475}{0.98 \times 2 \times 0.823 \times 0.90}=2222 \mathrm{veh} / \mathrm{h}
\end{gathered}
$$

Paso 5: Estimar la velocidad y densidad

Se calcularon dos valores de velocidad y densidad, uno para la gestión presente y otra para las condiciones a futuras establecidas. La tabla 8 se utilizará para estimar velocidades. La misma ecuación se aplica a ambos casos, además de un mismo FFS con un flujo sobre los $1400 \mathrm{veh} / \mathrm{h} / \mathrm{c}$.

$$
\begin{gathered}
\mathrm{S}(\text { Presente })=65-0.00001418(\mathrm{Vp}-1.400)^{2} \\
\mathrm{~S}(\text { Presente })=61.7 \mathrm{mi} / \mathrm{hr} \\
\mathrm{S}(\text { Futuro })=65-0.00001418(\mathrm{Vf}-1.400)^{2} \\
\mathrm{~S}(\text { Futuro })=55.40 \mathrm{mi} / \mathrm{hr}
\end{gathered}
$$

Luego pasamos a calcular las demandas correspondientes según los eventos correspondientes.

$$
\begin{gathered}
D=\frac{v p}{S} \\
\text { D (presente) }=\frac{1886}{61.7}=31 \mathrm{veh} / \mathrm{mi} / \mathrm{ln} \\
\mathrm{D}(\text { futuro })=\frac{2222}{55.40}=40 \mathrm{veh} / \mathrm{mi} / \mathrm{ln}
\end{gathered}
$$


Paso 6: Determinar Niveles de servicio

De la tabla 9, los niveles de servicio de la situación con la mejora es $\mathrm{D}$, y el nivel de servicio para el escenario a futuro con la mejora es E.

En el Anexo 3 y 4 del presente proyecto se adjunta todo el procedimiento y resultado de los demás puntos de aforo tanto para Niveles de servicio actual y Niveles de servicio con la gestión de la reversibilidad y proyección futura respectivamente.

\subsection{ANÁLISIS DEL PROCESAMIENTO DE DATOS}

En la siguiente tabla $\mathrm{N}^{\circ} 18$ tenemos los valores principales del aforo vehicular del mes de noviembre (martes y jueves). Volumen de máxima demanda (Veh/h), factor de hora pico(FHP), velocidad de flujo libre (mi/hr), volumen de ajuste vehicular(Veh/h), factor de camiones pesados(Fhv), velocidad (mi/hr) y densidad (veh/mi/ln). Los cuales fueron de fundamental requisito para poder hallar los niveles de servicios actuales.

Tabla 18:Resumen Niveles de Servicio Actual (Nov 2016)

\begin{tabular}{|c|c|c|c|c|c|c|c|c|c|c|c|}
\hline \multicolumn{12}{|c|}{ RESUMEN NIVELES DE SERVICIO ACTUAL (Nov 2016) } \\
\hline \multirow{2}{*}{\multicolumn{2}{|c|}{ AFORO VEHICULAR }} & Punto de Aforo & Volumen & FHP & FFS & $V_{p}$ & Fhv & Flujo & Velocidad & \multirow{2}{*}{ Densidad } & \multirow{2}{*}{ Nivel de servicio } \\
\hline & & & veh/ $/ \mathrm{hr}$ & - & $\mathrm{mi} / \mathrm{hr}$ & veh/hr & - & $\mathrm{veh} / \mathrm{h} / \mathrm{ln}$ & $\mathrm{mi} / \mathrm{hr}$ & & \\
\hline MARTES & SUR - NORTE & 1 & 2736.00 & 0.98 & 65 & 1256 & 0.823 & 1400.00 & 64.70 & 19 & C \\
\hline MARTES & NORTE - SUR & 1 & 6879.00 & 0.98 & 65 & 3095 & 0.833 & 1400.00 & 27.71 & 112 & $F$ \\
\hline MARTES & SUR-NORTE & 2 & 1938.00 & 0.99 & 65 & 915 & 0.833 & 1400.00 & 35.58 & 26 & D \\
\hline MARTES & NORTE - SUR & 2 & 7362.00 & 0.95 & 65 & 3286 & 0.833 & 1400.00 & 15.65 & 210 & $F$ \\
\hline & & & & & & & & & & & \\
\hline JUEVES & SUR - NORTE & 1 & 2732.00 & 0.98 & 65 & 1235 & 0.823 & 1400.00 & 53.14 & 23 & c \\
\hline JUEVES & NORTE - SUR & 1 & 6350.00 & 0.91 & 65 & 3062 & 0.805 & 1400.00 & 30.35 & 101 & $F$ \\
\hline JUEVES & SUR - NORTE & 2 & 1860.00 & 0.98 & 65 & 918 & 0.823 & 1400.00 & 33.91 & 27 & $D$ \\
\hline JUEVES & NORTE - SUR & 2 & 6902.00 & 0.96 & 65 & 3132 & 0.811 & 1400.00 & 26.04 & 120 & $F$ \\
\hline
\end{tabular}

En la tabla 19 se muestra los valores para hallar los niveles de servicio con la gestión de reversibilidad, cabe resaltar que, al aplicar la reversibilidad, el tramo de Sur - Norte tiene 2 carriles a comparación del tramo Norte - Sur que se define con 4 carriles. Haciendo un comparativo en los Niveles de servicio de la tabla 18 podemos apreciar que en el tramo de Norte a Sur la densidad vehicular y los niveles de servicio mejoran a raíz del carril adicional que la gestión aplica. 
Tabla 19: Resumen de Niveles de Servicio con Reversibilidad

\begin{tabular}{|c|c|c|c|c|c|c|c|c|c|c|c|}
\hline \multicolumn{12}{|c|}{ RESUMEN NIVELES DE SERVICIO CON REVERSIBILIDAD (Nov 2016) } \\
\hline \multirow{2}{*}{\multicolumn{2}{|c|}{ AFOROS VEHICULARES }} & Punto de Aforo & Volumen & FHP & FFS & $\mathrm{Vp}$ & Fhv & Flujo & Velocidad & Densidad & \multirow{2}{*}{ Nivel de servicio } \\
\hline & & & $\mathrm{veh} / \mathrm{hr}$ & - & $\mathrm{mi} / \mathrm{hr}$ & $\mathrm{veh} / \mathrm{hr}$ & - & veh/h/ln & $\mathrm{mi} / \mathrm{hr}$ & $\mathrm{veh} / \mathrm{mi} / \mathrm{ln}$ & \\
\hline MARTES & SUR - NORTE & 1 & 2736 & 0.98 & 65.00 & 1886 & 0.82 & 1400 & 61.70 & 31 & $D$ \\
\hline MARTES & NORTE - SUR & 1 & 6879 & 0.98 & 65.00 & 2302 & 0.83 & 1400 & 51.58 & 42 & $\mathrm{E}$ \\
\hline MARTES & SUR - NORTE & 2 & 1938 & 0.99 & 65.00 & 1246 & 0.83 & 1400 & 45.34 & 26 & C \\
\hline MARTES & NORTE - SUR & 2 & 7362 & 0.95 & 65.00 & 2465 & 0.83 & 1400 & 48.20 & 48 & $\mathrm{~F}$ \\
\hline & & & & & & & & & & & \\
\hline JUEVES & SUR - NORTE & 1 & 2732 & 0.98 & 65.00 & 1852 & 0.82 & 1400 & 54.93 & 32 & D \\
\hline JUEVES & NORTE - SUR & 1 & 6350 & 0.91 & 65.00 & 2297 & 0.81 & 1400 & 51.68 & 42 & $E$ \\
\hline JUEVES & SUR - NORTE & 2 & 1860 & 0.98 & 65.00 & 1222 & 0.82 & 1400 & 44.66 & 26 & C \\
\hline JUEVES & NORTE - SUR & 2 & 6902 & 0.96 & 65.00 & 2349 & 0.81 & 1400 & 50.72 & 44 & $E$ \\
\hline
\end{tabular}

La tabla 20 muestra el resumen del nivel de servicio aplicando la gestión de reversibilidad de carril en una proyección de 5 años, esta proyección se hace con un gráfico exponencial de proyección del parque automotor de Lima y Callao de los últimos años. En los resultados obtenidos vemos que el parque automotor para el año 2022 aumentará en un $27 \%$ en comparación con los resultados actuales. A pesar de este Aumento considerable y de tener un Nivel de Servicio F, se puede apreciar que las densidades con la gestión a futuro del tramo NORTE - SUR siguen siendo menores que las densidades de dicho tramo, pero sin la gestión aplicada.

Tabla 20: Resumen de Niveles de servicio con Reversibilidad a Proyección Futura

\begin{tabular}{|c|c|c|c|c|c|c|c|c|c|c|c|}
\hline \multicolumn{12}{|c|}{ RESUMEN NIVELES DE SERVICIO CON REVERSIBILIDAD A PROYECCIÓN FUTURA (Nov 2016) } \\
\hline \multirow{2}{*}{\multicolumn{2}{|c|}{ AFOROS VEHICULARES }} & Punto de Aforo & Volumen & FHP & FFS & $V_{p}$ & Fhv & Flujo & Velocidad & Densidad & \multirow{3}{*}{ Nivel de servicio } \\
\hline & & & veh/hr & $\cdot$ & $\mathrm{mi} / \mathrm{hr}$ & veh/hr & $\cdot$ & veh/h/ln & $\mathrm{mi} / \mathrm{hr}$ & $\mathrm{veh} / \mathrm{mi} / \mathrm{ln}$ & \\
\hline MARTES & SUR - NORTE & 1 & 3475 & 0.98 & 65.00 & 2222 & 0.82 & 1400 & 55.40 & 40 & \\
\hline MARTES & NORTE - SUR & 1 & 8736 & 0.98 & 65.00 & 2759 & 0.83 & 1400 & 32.30 & 85 & $F$ \\
\hline MARTES & SUR - NORTE & 2 & 2461 & 0.99 & 65.00 & 1492 & 0.83 & 1400 & 52.65 & 28 & D \\
\hline MARTES & NORTE - SUR & 2 & 9350 & 0.95 & 65.00 & 2953 & 0.83 & 1400 & 22.20 & 133 & $F$ \\
\hline JUEVES & SUR - NORTE & 1 & 3470 & 0.98 & 65.00 & 2219 & 0.82 & 1400 & 50.67 & 44 & $\bar{E}$ \\
\hline JUEVES & NORTE - SUR & 1 & 8065 & 0.91 & 65.00 & 2752 & 0.81 & 1400 & 32.64 & 84 & $F$ \\
\hline JUEVES & SUR - NORTE & 2 & 2362 & 0.98 & 65.00 & 1464 & 0.82 & 1400 & 52.22 & 28 & D \\
\hline JUEVES & NORTE - SUR & 2 & 8766 & 0.96 & 65.00 & 2815 & 0.81 & 1400 & 29.59 & 95 & $F$ \\
\hline
\end{tabular}

En la tabla $\mathrm{N}^{\circ} 21$ tenemos las densidades eventuales de nuestro caso de estudio de acuerdo a cada punto de aforo. Hacemos un comparativo entre la densidad actual contra la densidad de la mejora y también las densidades con las mejoras tanto actuales como futuras dentro de 5 años. Esto con la finalidad de verificar si hay una mejora de densidad a aparte de los niveles de servicio. 
Tabla 21: Porcentaje de incremento o decremento de la densidad del tránsito con los distintos eventos

\begin{tabular}{|c|c|c|c|c|c|c|c|c|}
\hline & & & \multicolumn{5}{|c|}{ Comparativo de las densidades y LOS en la actualidad vs la mejora } & \multirow{3}{*}{ AUMENTA } \\
\hline \multicolumn{2}{|c|}{ AFOROS VEHICULARES } & \multirow{2}{*}{$\begin{array}{c}\begin{array}{c}\text { Punto de } \\
\text { Aforo }\end{array} \\
1\end{array}$} & \multirow{2}{*}{$\begin{array}{c}\text { densidad actual } \\
19 \\
\end{array}$} & \multirow{2}{*}{$\frac{\text { NS }}{\mathrm{C}}$} & \multirow{2}{*}{$\begin{array}{c}\text { densidad con la } \\
\text { mejora }\end{array}$} & \multirow{2}{*}{$\begin{array}{l}\text { NS } \\
\text { D }\end{array}$} & \multirow{2}{*}{$\begin{array}{l}\% \\
60 \%\end{array}$} & \\
\hline MARTES & SUR-NORTE & & & & & & & \\
\hline MARTES & NORTE - SUR & 1 & 112 & $\mathrm{~F}$ & 42 & $\mathrm{E}$ & $-165 \%$ & DISMINUYE \\
\hline MARTES & SUR - NORTE & 2 & 26 & D & 26 & $\mathrm{C}$ & $-1 \%$ & AUMENTA \\
\hline MARTES & NORTE - SUR & 2 & 210 & $\mathrm{~F}$ & 48 & $\mathrm{~F}$ & $-335 \%$ & DISMINUYE \\
\hline JUEVES & SUR - NORTE & 1 & 23 & $\mathrm{C}$ & 32 & D & $37 \%$ & AUMENTA \\
\hline JUEVES & NORTE - SUR & 1 & 101 & $F$ & 42 & $E$ & $141 \%$ & DISMINUYE \\
\hline JUEVES & SUR - NORTE & 2 & 27 & D & 26 & C & $5 \%$ & AUMENTA \\
\hline JUEVES & NORTE - SUR & 2 & 120 & $\mathrm{~F}$ & 44 & $E$ & $175 \%$ & DISMINUYE \\
\hline
\end{tabular}

\begin{tabular}{|c|c|c|c|c|c|c|c|}
\hline & & \multirow[b]{3}{*}{$\begin{array}{l}\text { Punto de } \\
\text { Aforo }\end{array}$} & \multicolumn{5}{|c|}{$\begin{array}{l}\text { PROYECCÓN DELTRANSITO CON EL PARQUE AUTOMOTOR DE UMA - } \\
\text { CALAO }\end{array}$} \\
\hline & & & \multicolumn{5}{|c|}{ Con proyección a futuro (Dentro de 5 años) } \\
\hline \multicolumn{2}{|c|}{ AFOROS VEHICULARES } & & $\begin{array}{c}\text { densidad con } \\
\text { mejora }\end{array}$ & NS & $\begin{array}{l}\text { densidad con } \\
\text { mejora futura }\end{array}$ & NS & $\%$ \\
\hline MARTES & SUR-NORTE & 1 & 31 & D & 40 & E & $29 \%$ \\
\hline MARTES & NORTE - SUR & 1 & 42 & $\mathbf{E}$ & 85 & $\mathrm{~F}$ & $103 \%$ \\
\hline MARTES & SUR-NORTE & 2 & 26 & C & 28 & D & $9 \%$ \\
\hline MARTES & NORTE - SUR & 2 & 48 & $\mathrm{~F}$ & 133 & $\mathrm{~F}$ & $176 \%$ \\
\hline JUEVES & SUR-NORTE & 1 & 32 & D & 44 & $E$ & $38 \%$ \\
\hline JUEVES & NORTE - SUR & 1 & 42 & $E$ & 84 & $\mathrm{~F}$ & $101 \%$ \\
\hline JUEVES & SUR-NORTE & 2 & 26 & C & 28 & D & $9 \%$ \\
\hline JUEVES & NORTE-SUR & 2 & 44 & $E$ & 95 & $\mathrm{~F}$ & $118 \%$ \\
\hline
\end{tabular}

En las siguientes barras de la imagen 37 y 38 se muestra a detalle la mejora de las densidades y niveles de servicio en los tramos que se le añade un carril adicional (Norte - Sur), formando cuatro carriles en total para dar fluidez al congestionamiento vehicular. Además, vemos que el tramo que queda con 2 carriles (Sur - Norte), las densidades y niveles de servicio aumentan, pero de manera moderada. 
Imagen 37: Densidad Actual vs Mejora (Nov/ día martes)

\section{Noviembre / Día Martes Densidad Actual Vs Mejora (veh/mi/ln)}
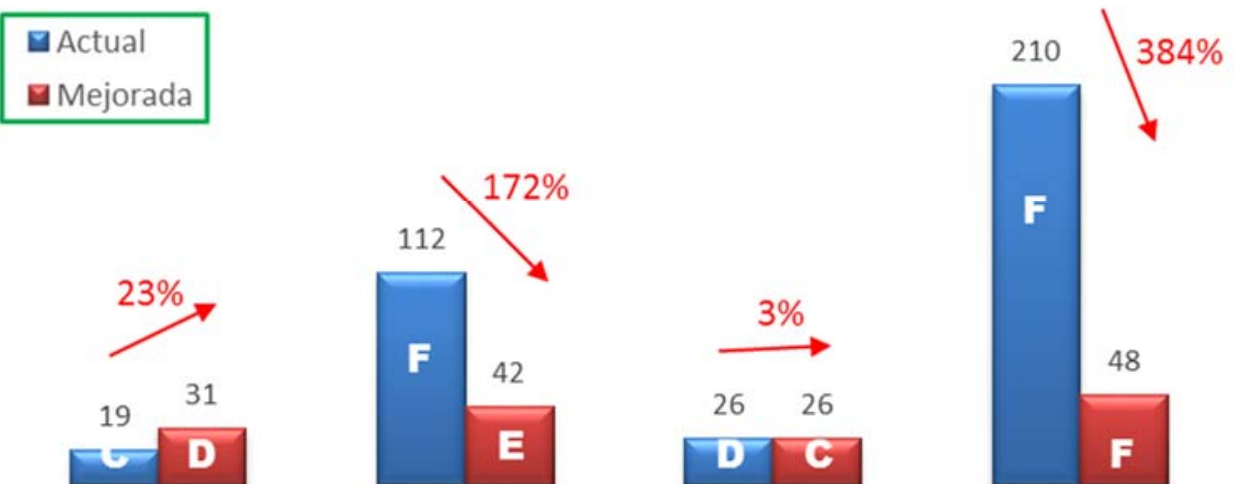

Puente Control

Puente Control Zarumilla / Sur - Norte Zarumilla / Norte - Sur

Avenida Tomas Valle / Avenida Tomas Valle / Sur - Norte Norte - Sur

En las barras comparativas de las densidades con mejora vs con la densidad mejorada proyectada vemos un gran cambio en los niveles de servicio y las densidades (Ver imagen 39 y 40). Sin embargo, a pesar de que el nivel de servicio del tramo de cuatro carriles vuelve a un $\mathrm{F}$, las densidades proyectadas a 5 años siguen siendo menores a las densidades actuales.

Imagen 38: Densidad Actual vs Mejora (Nov/ día jueves)

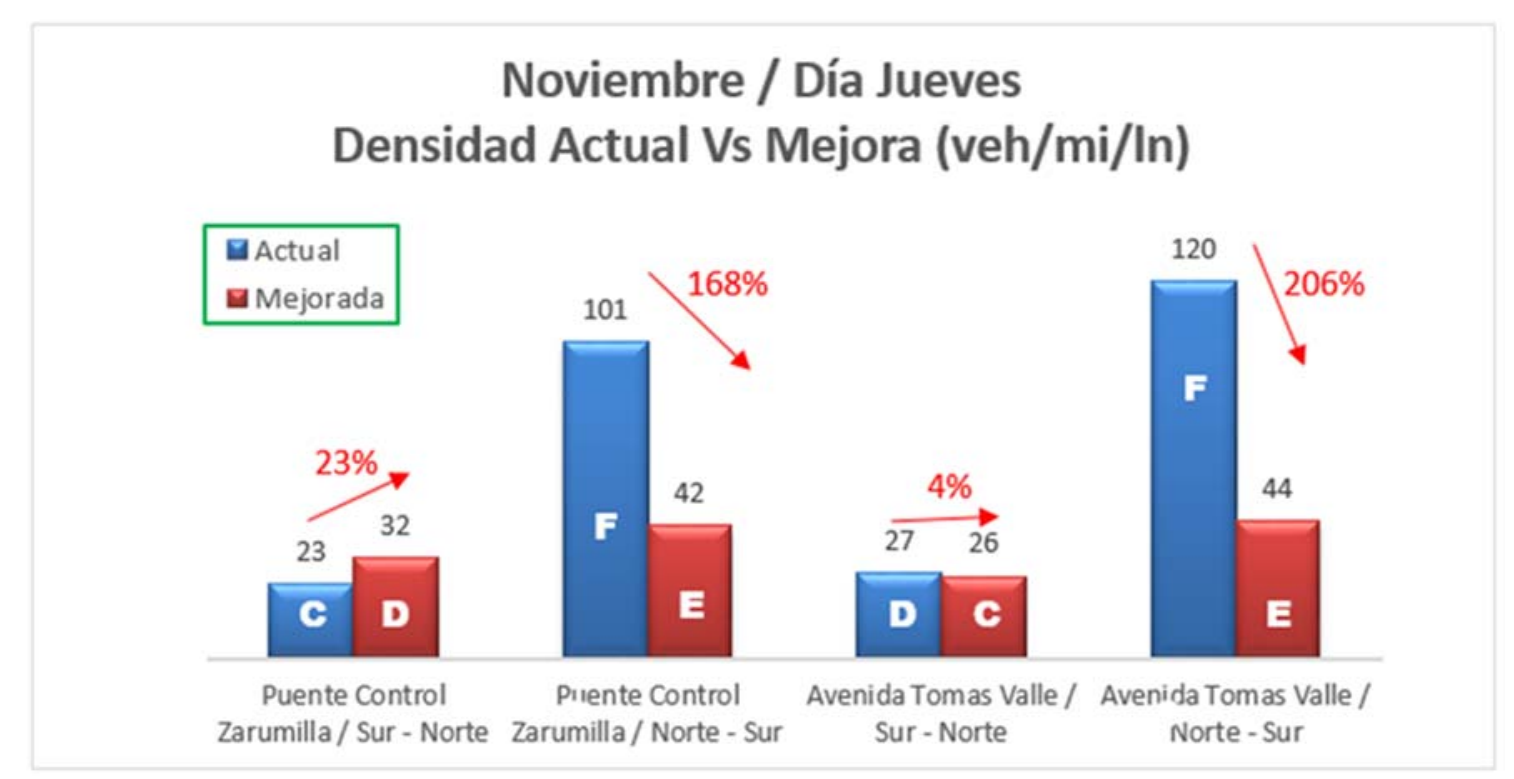

Imagen 39: Densidad Mejorada vs Densidad Proyectada (Nov/ día martes) 


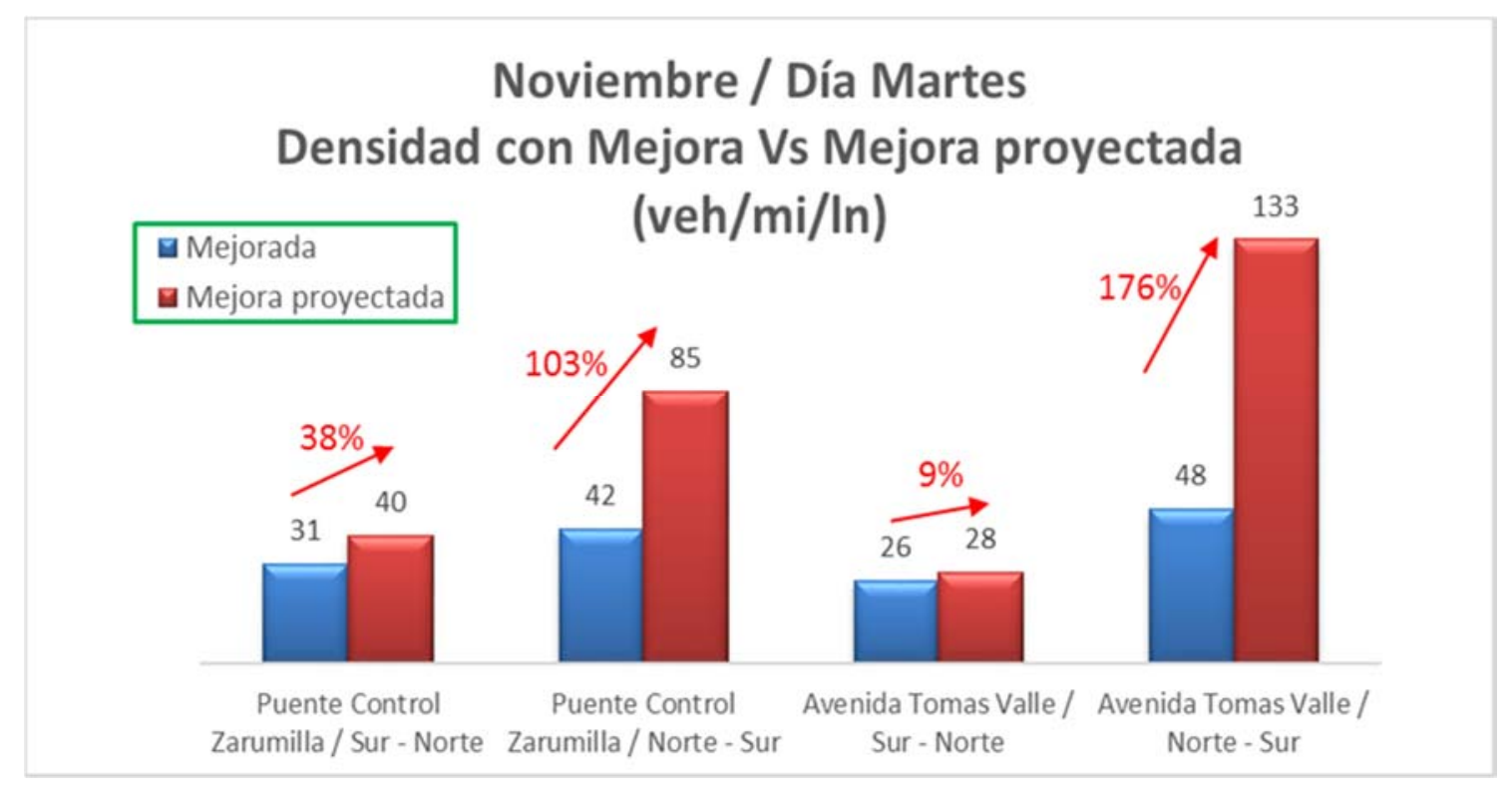

Imagen 40: Densidad Mejorada vs Densidad Proyectada (Nov/ día jueves)

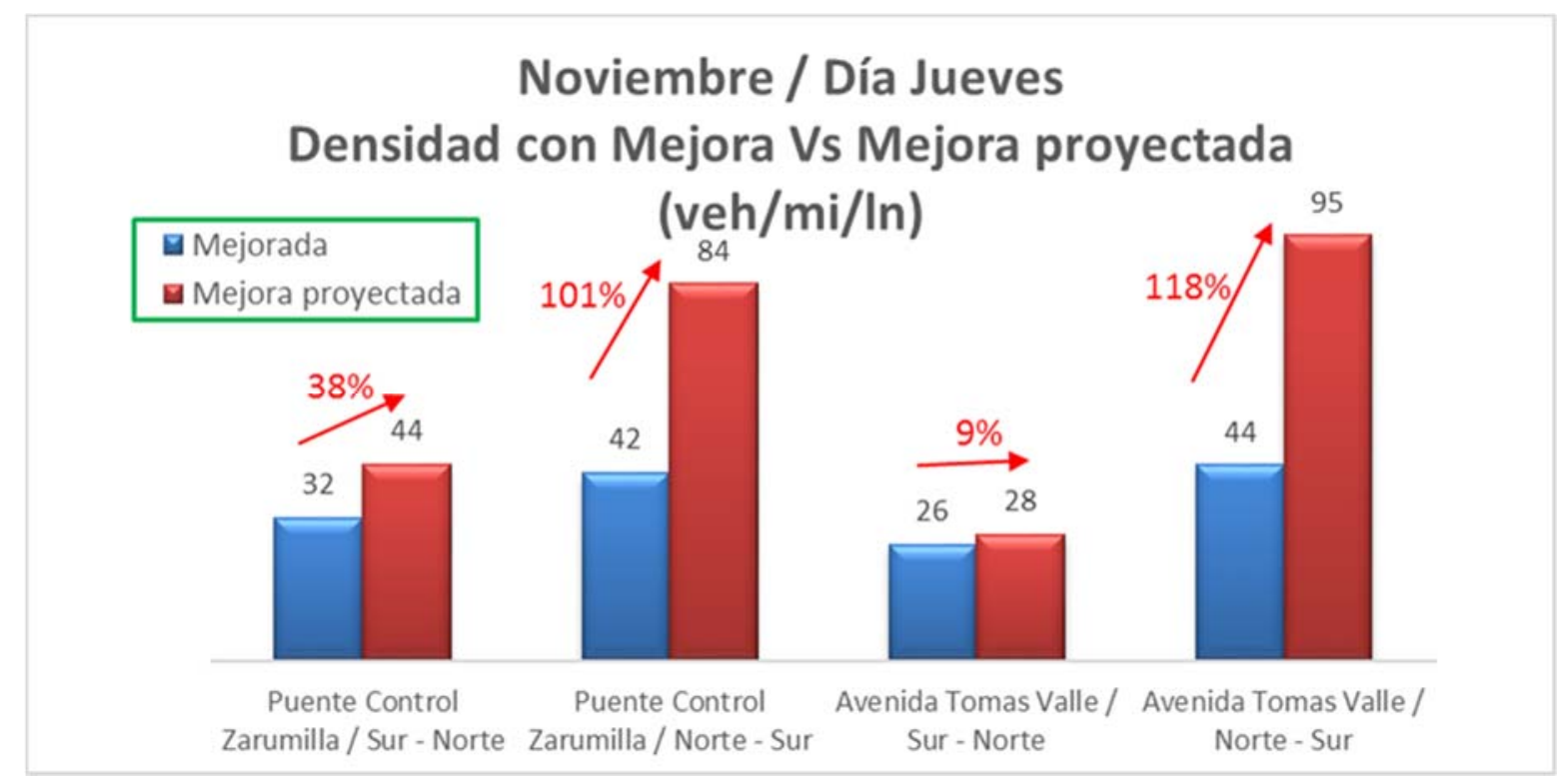

\subsection{ESTUDIO DE IMPACTO VIAL}

El presente trabajo de tesis tiene como objetivo realizar el desarrollo de un Estudio de Impacto Vial (EIV). El área inicial de estudio involucraba la arteria principal de la carretera panamericana norte, en los puntos del intercambio vial tomas valle hasta el puente control Zarumilla estos puntos pertenecientes al distrito de San Martin de Porras. Este estudio de impacto vial se basa en los principios desarrollados por Wardrop. 


\subsection{1 Área de influencia}

Para realizar un estudio de Impacto Vial es necesario primero reconocer y definir las vías aledañas a la arteria principal, dándoles alternativas de viajes a los transportistas la cual mitigue la densidad del parque automotor. A continuación, en la imagen 41 se ha identificado las vías alternas que generan menor congestionamiento vehicular entre los tramos de tomas valle y el puente control Zarumilla

En el tramo de Sur a Norte la ruta a seguir será: entrar por el Jr. Alicia Alarcon, seguir por Av. Isidro Alcibar, continuar por Jr. Miguel Fort y salir a la Av. Eduardo de Habich, posteriormente dirigirse por toda la Av. Tupac Amaru hasta llegar a la Av. Tomas Valle seguir en dirección al ovalo y tomar la primera salida a la Panamericana Norte.

En el tramo de Norte a Sur: Ingresando por la Tomas Valle virar a la derecha y tomar la Av. Alfredo Mendiola hasta el ovalo Eduardo de Habich, tomar la primera salida y dirigirse hasta la primera salida en la Av. Pierola, posteriormente tomar la Av. Aguilar hasta la Av. Zarumilla la cual tiene como salida al Puente Control Zarumilla.

Estos desvíos de rutas alternas se han tenido en consideración con el tiempo de llegada más próxima entre los puntos de Tomas Valle y el Puente Control Zarumilla, tal como se muestra en la imagen 41 a continuación. La ruta alterna de Sur a Norte está representada por una línea verde, mientras que la ruta alterna de Norte a Sur está representada por una línea roja. El tramo de estudio donde se aplicará la reversibilidad de carril está comprendido desde el intercambio vial Tomas Valle y el Puente Control Zarumilla la cual se representará con una línea color magenta. 
Imagen 41: Rutas alternas a la vía donde se aplicará la reversibilidad de carril

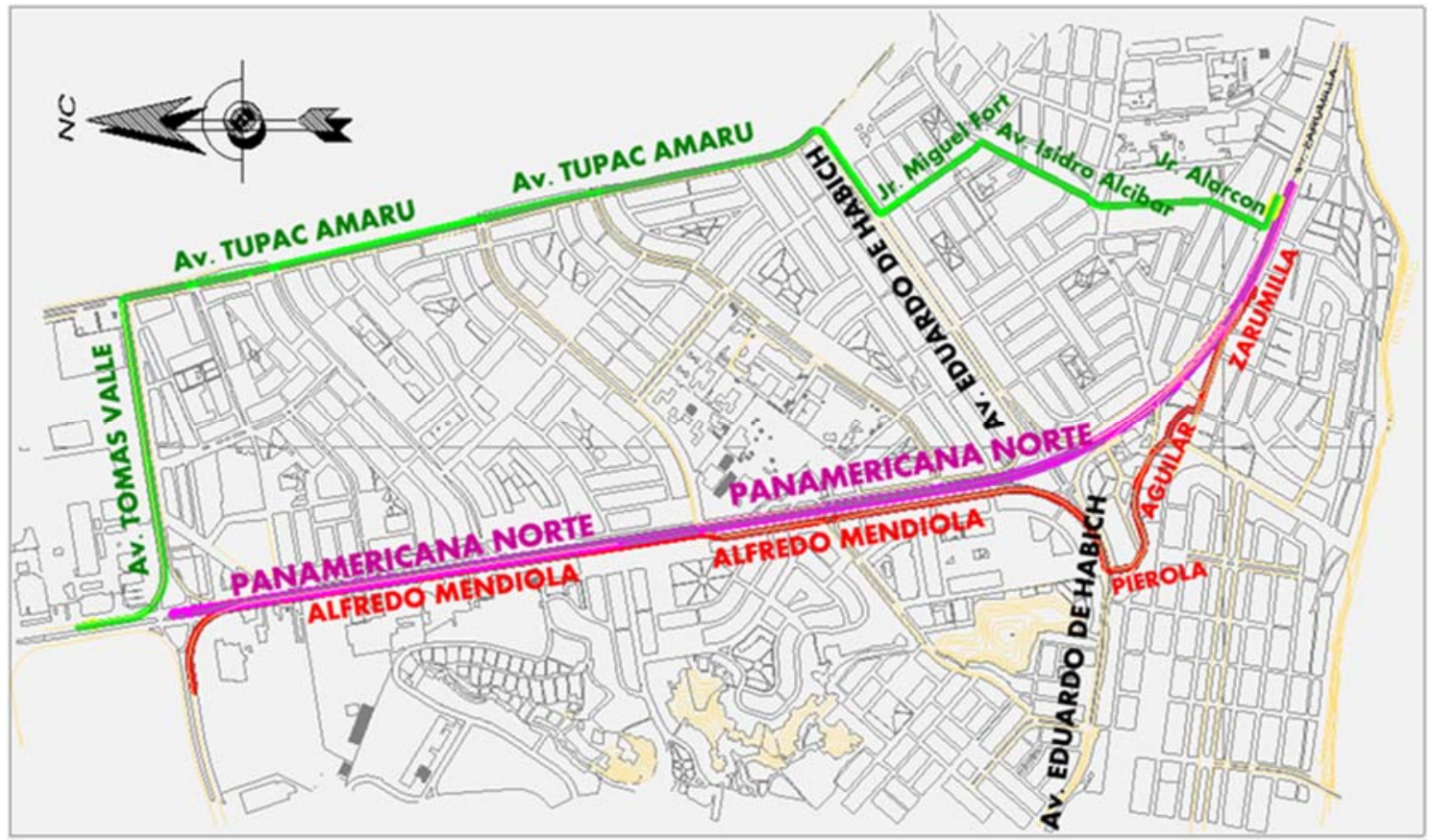

Fuente: Propio 


\section{CAPITULO VI: VALIDACIÓN DEL PROYECTO}

\subsection{CREACIÓN DE LA RED VIAL DEL CASO DE ESTUDIO}

Como complemento del proyecto de tesis, se realizará una guía del uso de la plataforma Aimsun 8.02 adecuado y aplicado a nuestro caso de estudio para el análisis a nivel mesoscópico, para finalizar se establecerá una serie de conclusiones y recomendaciones.

\subsubsection{Importar base cartográfica desde Open Street Maps}

Una vez abierta el software, se selecciona nuevo proyecto y se escoge la plantilla "002 TSS Template ESP", nos ubicamos dentro de la zona que deseamos importar a nivel mesoscópico: país, provincia, cuidad, sector, desactivamos las opciones de "micro" "macro" y "estático", luego en la barra de herramientas inferior seleccionamos "importar desde internet" e inmediatamente pulsamos la opción "crear". Luego de unos segundos de que se realizó el proceso anterior, el programa indica un cuadro de dialogo para el "importador de Open Stret Maps - OSM", seleccionamos las opciones de "importar carreteras: como una red", aceptamos y luego se realiza la importación de la red vial de la zona del distrito de San Martín de Porres acotando el caso de estudio, que se encuentra en la arteria principal de la Panamericana Norte entre los intercambios viales Tomas Valle y Eduardo de Habich, tal como se aprecia en la Imagen 42. 
Imagen 42: Red Vial del distrito de San Martín de Porres en OSM

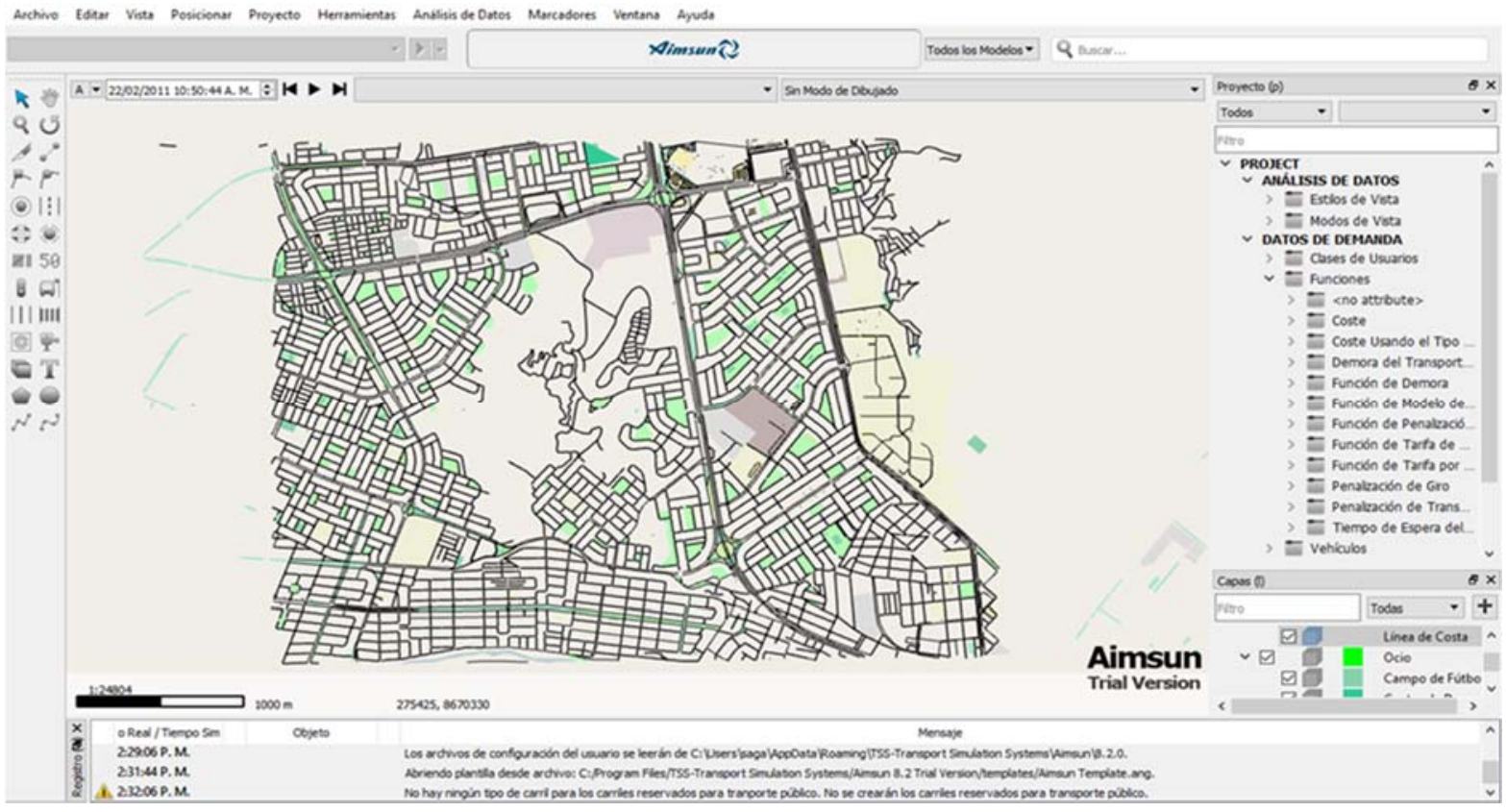

\subsubsection{Creación de Secciones:}

Una vez realizado el paso anterior para importar la red vial, desde OSM, procedemos a realizar las secciones de nuestro caso de estudio con las redes adecuadas, anchos de carriles, intersecciones, pasos a desnivel. Para ello utilizamos las opciones localizadas en la barra de trabajo izquierda.

Imagen 43: Realización de secciones de nuestro caso de estudio

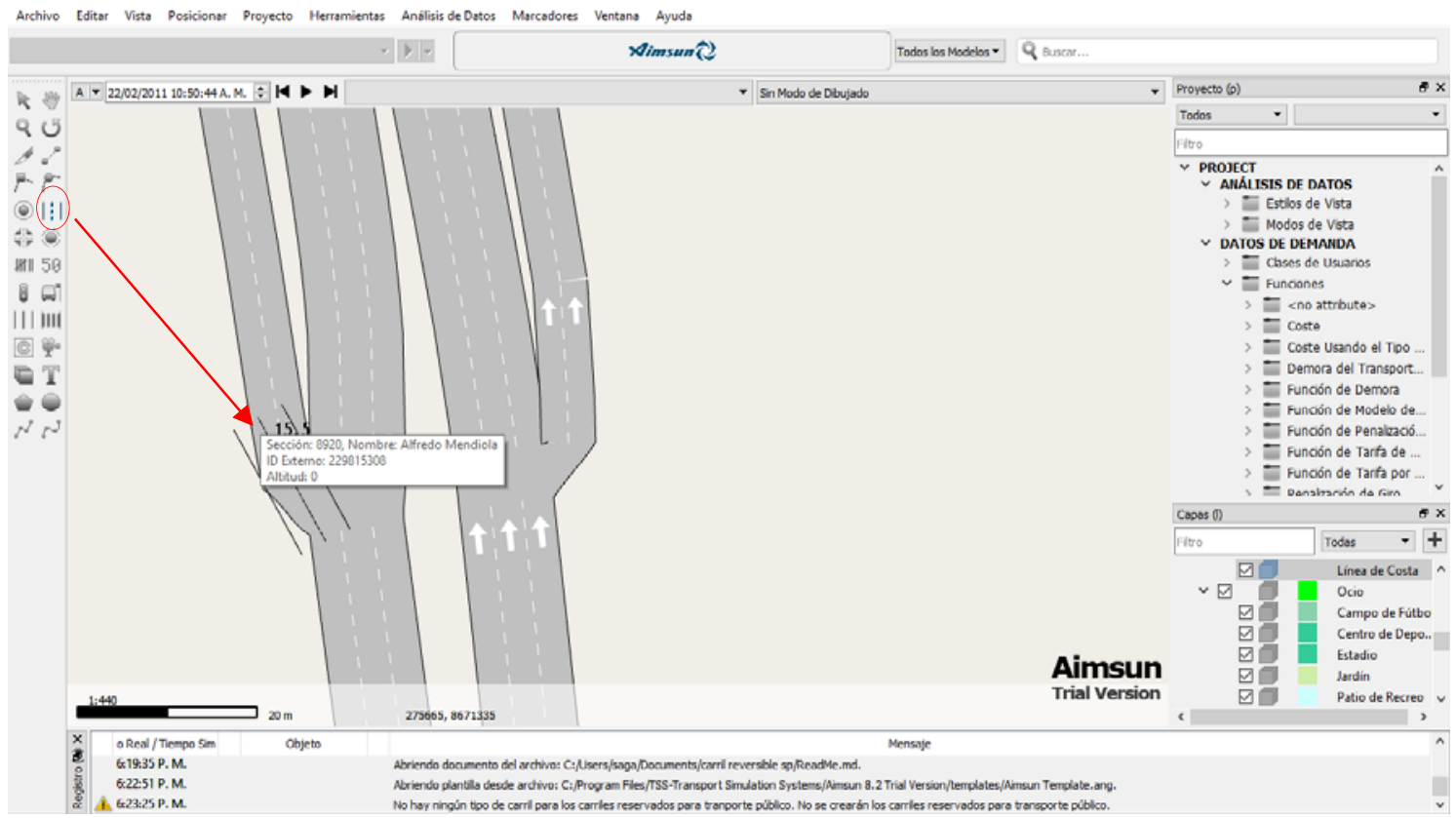


En la imagen 43, se indicará como realizar las secciones utilizando como referencia una imagen importada desde Autocad sobre la cual se dibujará la red para modelar nuestro caso de estudio, para lo cual se utilizó la opción "Crear una sección". Como se puede observar en la imagen anterior, no solo se puede construir carriles de vías rectas, se pueden realizar: rotondas o redondeles, pasos a desnivel elevados, curvas de acceso, distribuidores. para ello es necesario utilizar las opciones de trazo ubicadas en la barra lateral izquierda.

Luego de creadas todas las secciones, procedemos a realizar las uniones de las mismas para indicar a la herramienta informática cuales son las vías conectadas, para ello se utiliza la opción "Crear una conexión entre dos objetos", es importante tener en cuenta los sentidos de circulación para ello damos click sobre la sección origen y mantenemos pulsado hasta posicionarnos sobre la sección destino donde dejamos de dar click.

Si se tienes más de dos carriles y sentidos de circulación que se conectan como en nuestro caso de estudio, lo mejor es dar click dentro de la intersección "NODO" y realizar todas las uniones con el nodo seleccionado, así la herramienta informática entiende que es una intersección.

\subsubsection{Creación de Nodos:}

Los nodos en la red vial, representan las intersecciones consideradas como un grupo de giros y se realiza desde el cuadro de dialogo del nodo y asignando los giros de unión entre los carriles uno a uno, tal como se indica en la siguiente figura. A continuación, en la Imagen 44 se ilustra toda la red Vial terminada: 
Imagen 44: Red vial definida de nuestro caso de Estudio

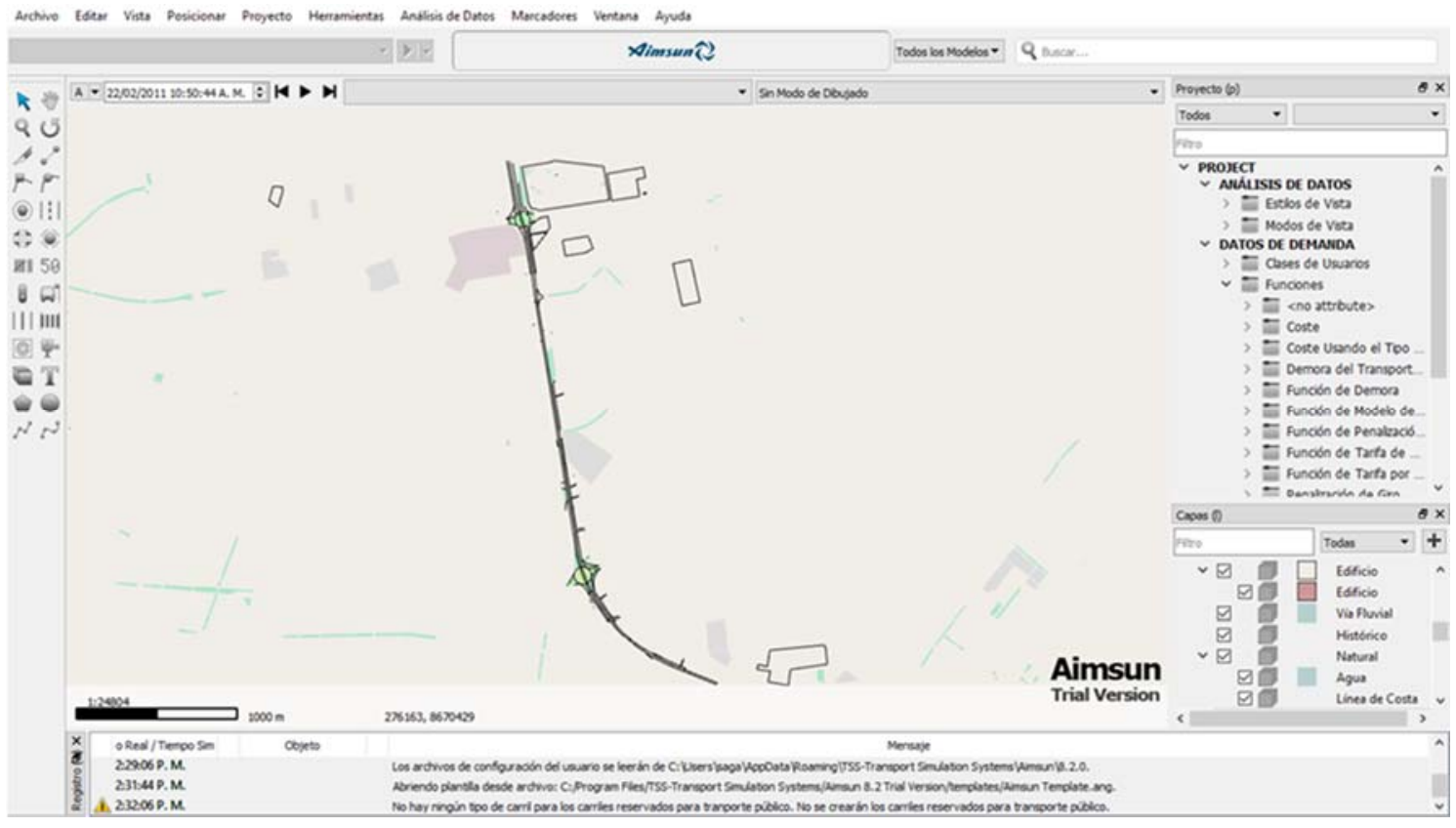

\subsubsection{Jerarquización Vial, ancho de carriles, número de carriles y velocidades:}

Una vez terminada la red vial es necesario definir su jerarquía, en función al tipo de vía, en nuestro caso de estudio existe 3 tipos de vías: autopista, carril secundario, rampa de entrada/salida , para definir la jerarquía seleccionamos la sección y damos click derecho y en la opción "tipo de vía" escogemos la deseada, destacamos que al escoger el tipo de vía por default se establece un ancho de carril y velocidad de circulación, pero se las puede modificar en función de los requerimientos particulares. El número de carriles se lo cambia en función a las necesidades, pero hay un método abreviado que consiste en seleccionar la sección y pulsar la tecla ctrl" número de carriles" por ejemplo para cuatro carriles en la sección se digita ctrl4. En la Imagen 45, 46 y 47 se muestra las jerarquías de vías del caso de estudio, así como la definición del ancho de carril y las velocidades de los tramos respectivamente. 
Imagen 45: Definiendo jerarquías de vía en nuestro caso de estudio

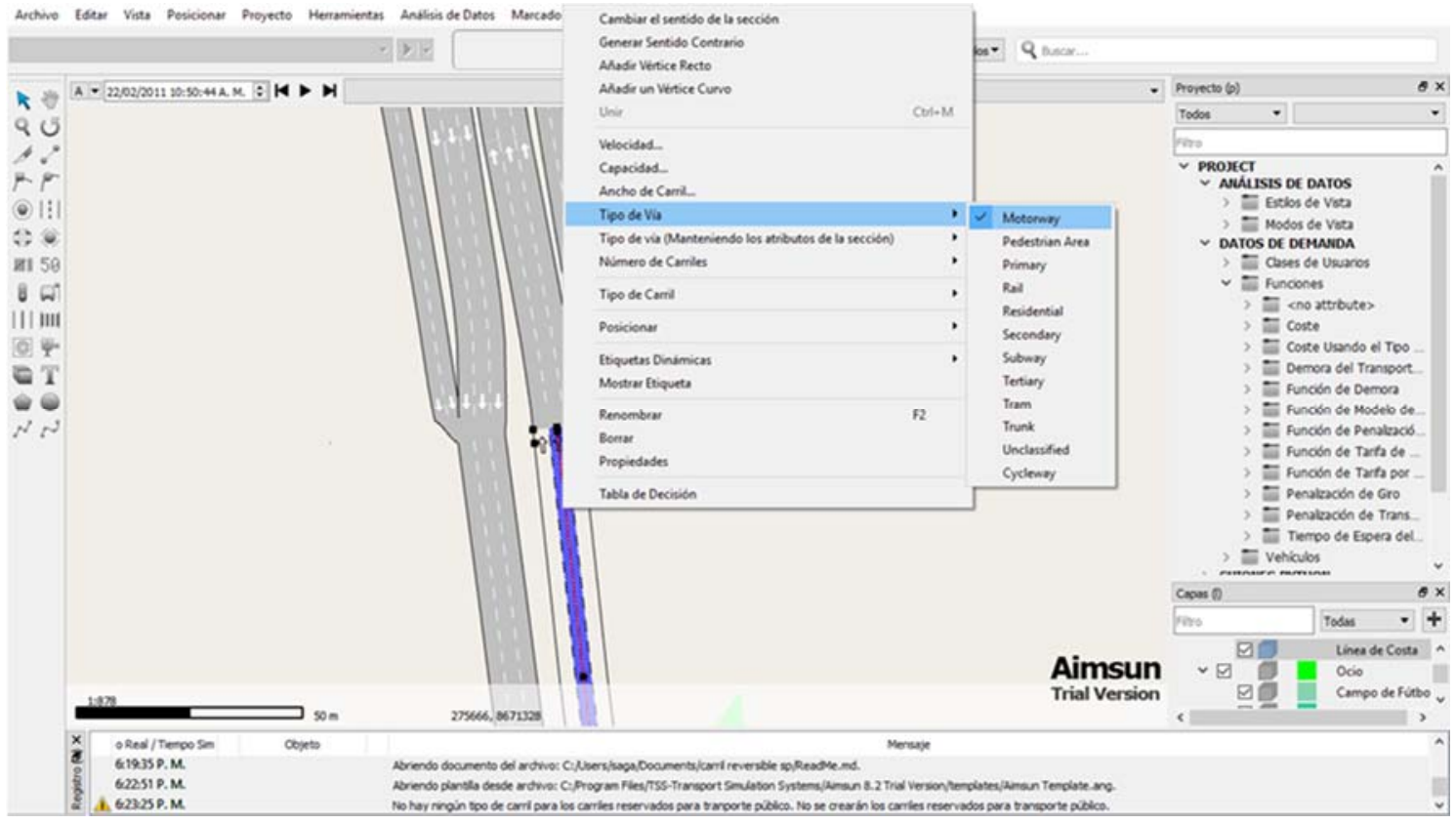

Imagen 46: Definiendo ancho de carril en las vías de nuestro caso de estudio

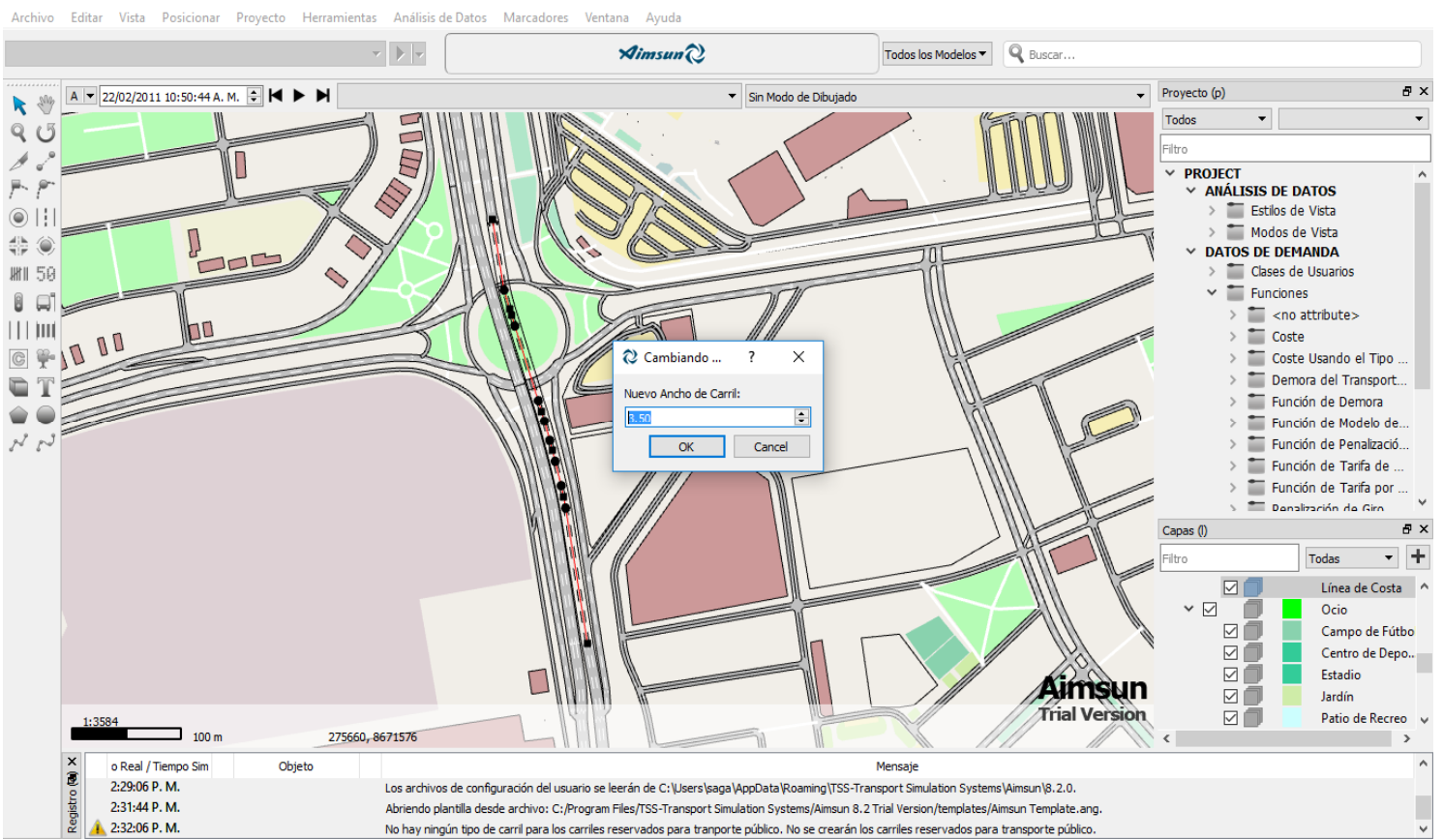


Imagen 47: Definiendo velocidades a las vías de nuestro caso de estudio

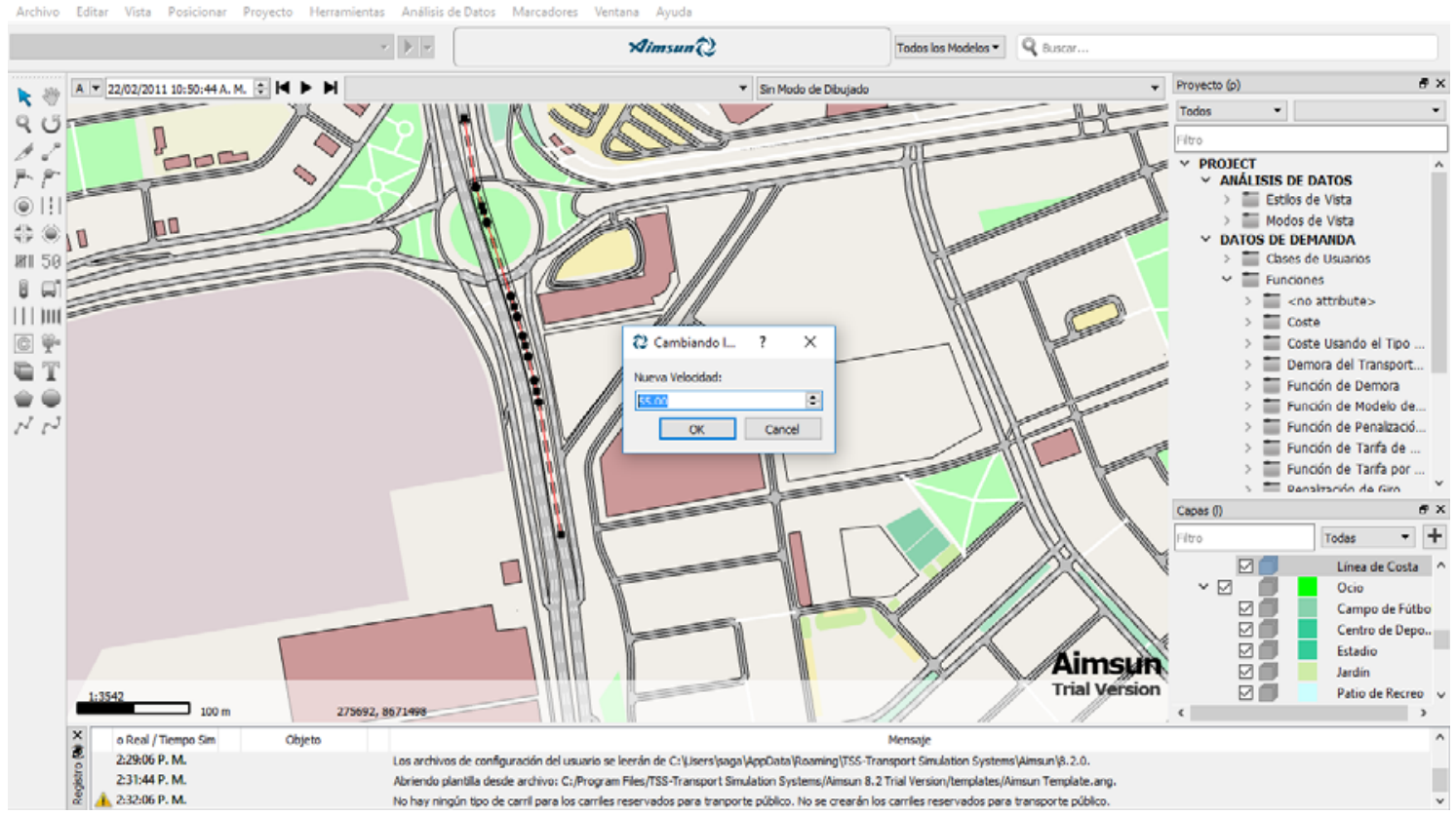

\subsection{ASIGNACIÓN DEL TRAFICO}

Una vez realizada la red, jerarquizada y acoplada en función de los requisitos de números, anchos y velocidades de los carriles de nuestro caso de estudio, es necesario asignar el tráfico que circulará por ella, para realizarlo existe el método de estado de tráfico, esta metodología sirve cuando hemos realizados aforos vehiculares, donde se han determinado los porcentajes de vehículos que ingresaron en la circulación de nuestro caso de estudio, y los tipos de vehículos que transitaron sobre el caso de estudio.

Por lo general la red se corre para las condiciones más desfavorables de circulación, ya que se asume que, si se soluciona los conflictos en la hora de máxima demanda o movimiento, se solucionará para las horas donde los flujos vehiculares son menores y por consecuencia los conflictos.

En el presente caso se ha identificado en el estudio de tráfico, que la hora de máximo movimiento en el tramo más congestionado en el aforo del día martes del mes de noviembre es de 9:15 - 10:15am, por lo que identificaremos a los estados de tráfico en función de su hora de análisis.

Para crear un "Estado de Tráfico" se realiza la siguiente ruta, en la barra de herramientas superior debemos hacer Proyecto- Nuevo-Datos de Demanda-Estado de Tráfico. 
Imagen 48: Estado del tráfico de acuerdo al tipo de vehículo.

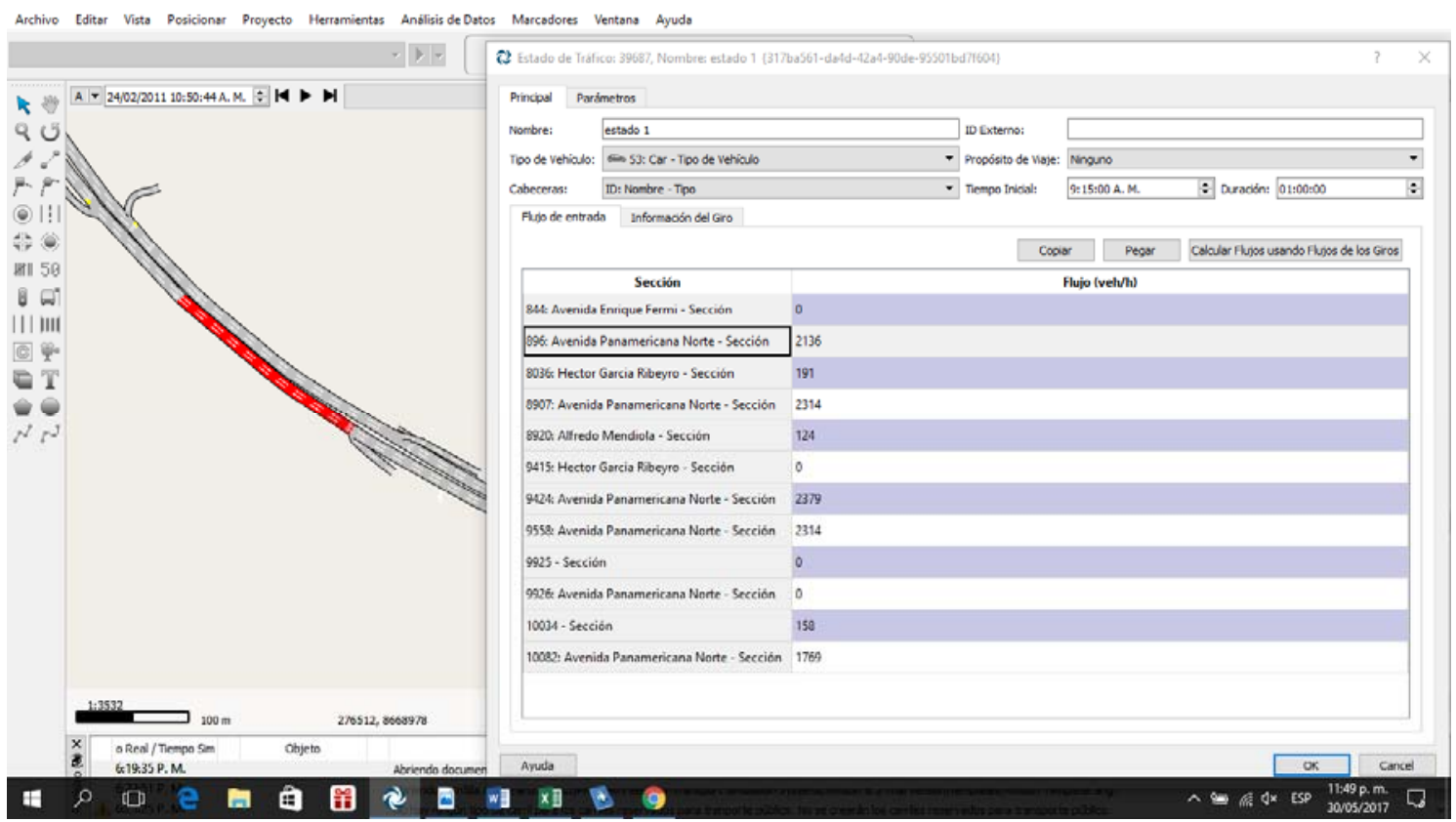

Tal como se muestra en la imagen 48, el estado de tráfico está relacionado con el tipo de vehículo, según el formato de aforo de nuestro caso de estudio se ha realizado tres estados uno para automóviles, otro para camiones y por último el de buses. Cada Estado de tráfíco está programado para la hora de máxima demanda es decir 9:15 - 10:15am.

En los diversos casos se consideran los porcentajes de flujos para cada giro, los cuales se registran en la opción "Flujo de entrada" en valores absolutos y en "Información del giro" los porcentajes de volumen en cada movimiento. Existe una opción de resaltar definiciones incorrectas.

\subsubsection{Demanda de tráfico para estados}

Con el objeto de generar una demanda de tráfico en función a los estados de tráfico que hemos creado, pulsamos botón derecho sobre "Demanda de Trafico" y seleccionamos crear demanda de tráfico, en el presente caso de estudio de nuestro proyecto hemos establecido dos demandas de tráfico, una para la hora de máximo movimiento $(100 \%$ de flujo máximo tal como se muestra en la imagen 49$)$ y otra para las horas valle $(60 \%$ del flujo máximo). Destacamos que es necesario colocar la hora de inicio: "9:15am”, el tiempo de duración: "1 hora", el tipo: "Estados" y el factor: 100\% en el caso de la demanda de hora pico, hecho esto procedemos a "añadir elemento de demanda" y cargamos los estados creados tanto para coches como para camiones. 
Imagen 49: Demanda de tráfico para nuestro caso de estudio

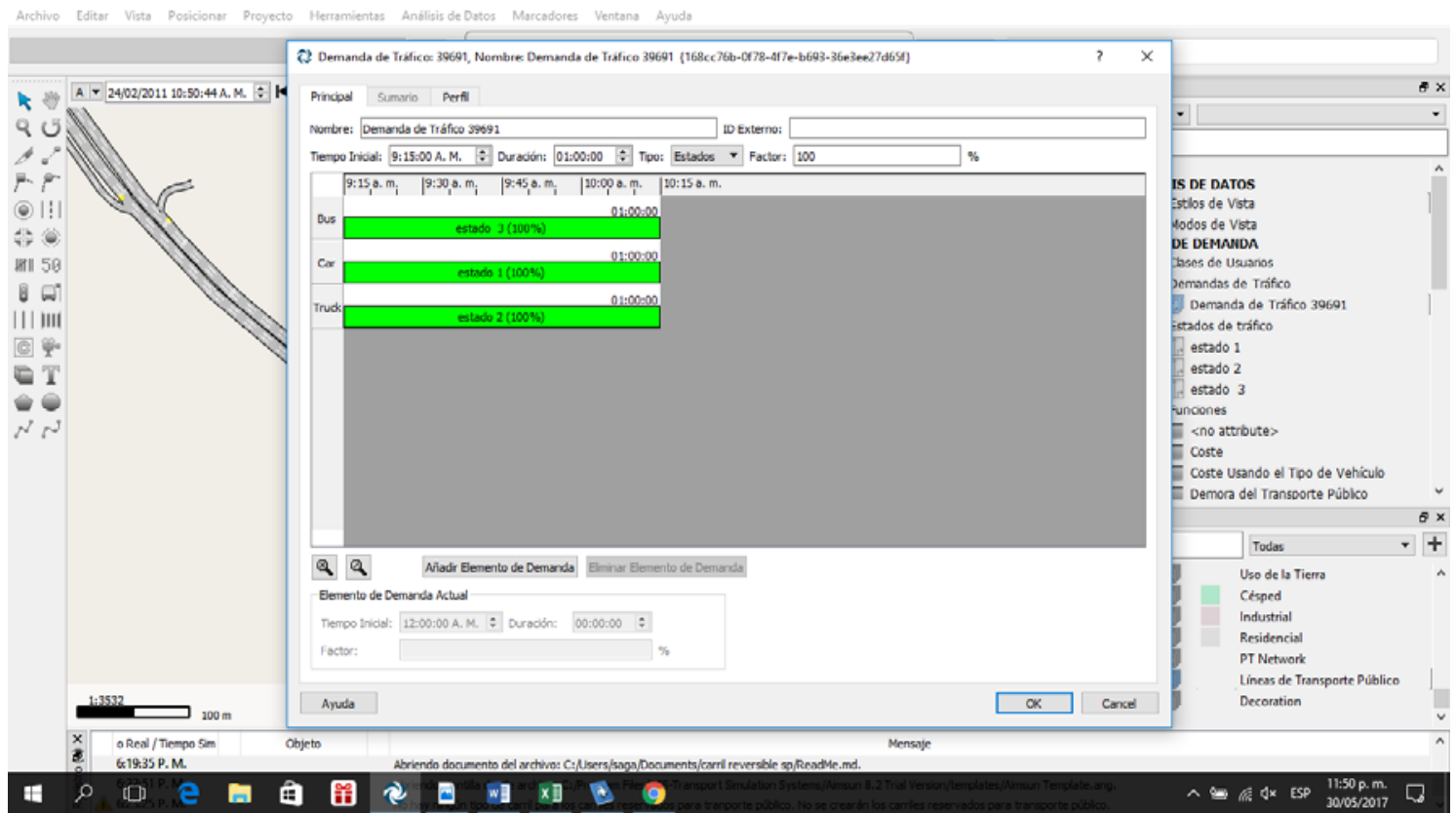

\subsubsection{Paraderos}

Antes de crear las líneas de transporte público debemos ubicar las paradas utilizando la opción "Crear una parada de transporte público" en la barra lateral izquierda. Tal como se aprecia en la imagen 50, Las paradas pueden ser normales o segregadas (desplazadas fuera de la vía), por default la parada es "tipo: normal”, pero para cambiarla a segregada se selecciona la parada y se da click derecho. 
Imagen 50: Ubicación de paraderos del transporte público en nuestro caso de estudio

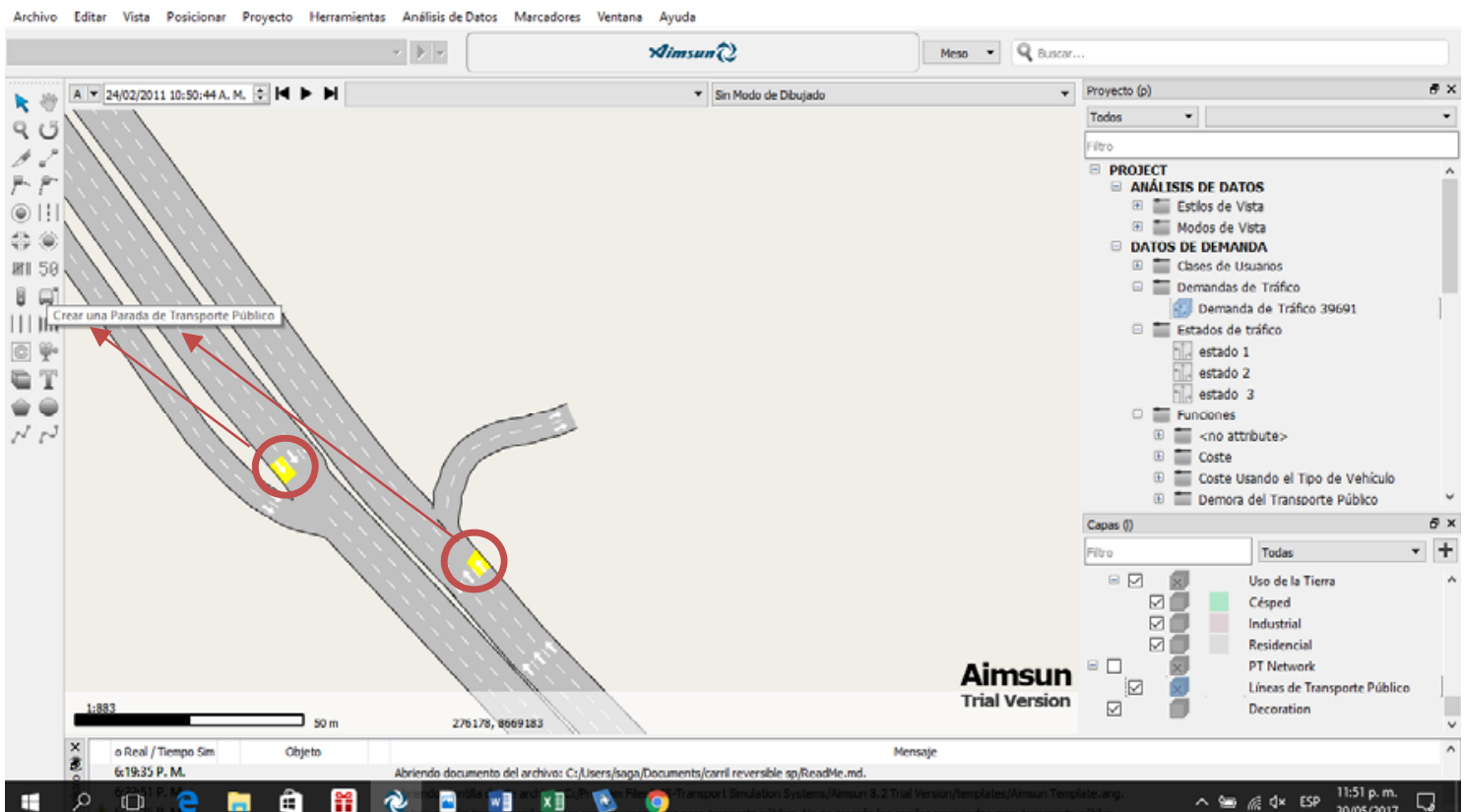

\subsubsection{Líneas de transporte público}

Sirven para modelar las líneas de transporte masivo o convencional que circulan por una determinada Red Vial, para realizar esto seguimos la siguiente ruta: Proyecto - Nuevo Transporte Público - Línea de transporte público, tal como se muestra en la imagen 51.

Imagen 51: modelación de las líneas de transporte público

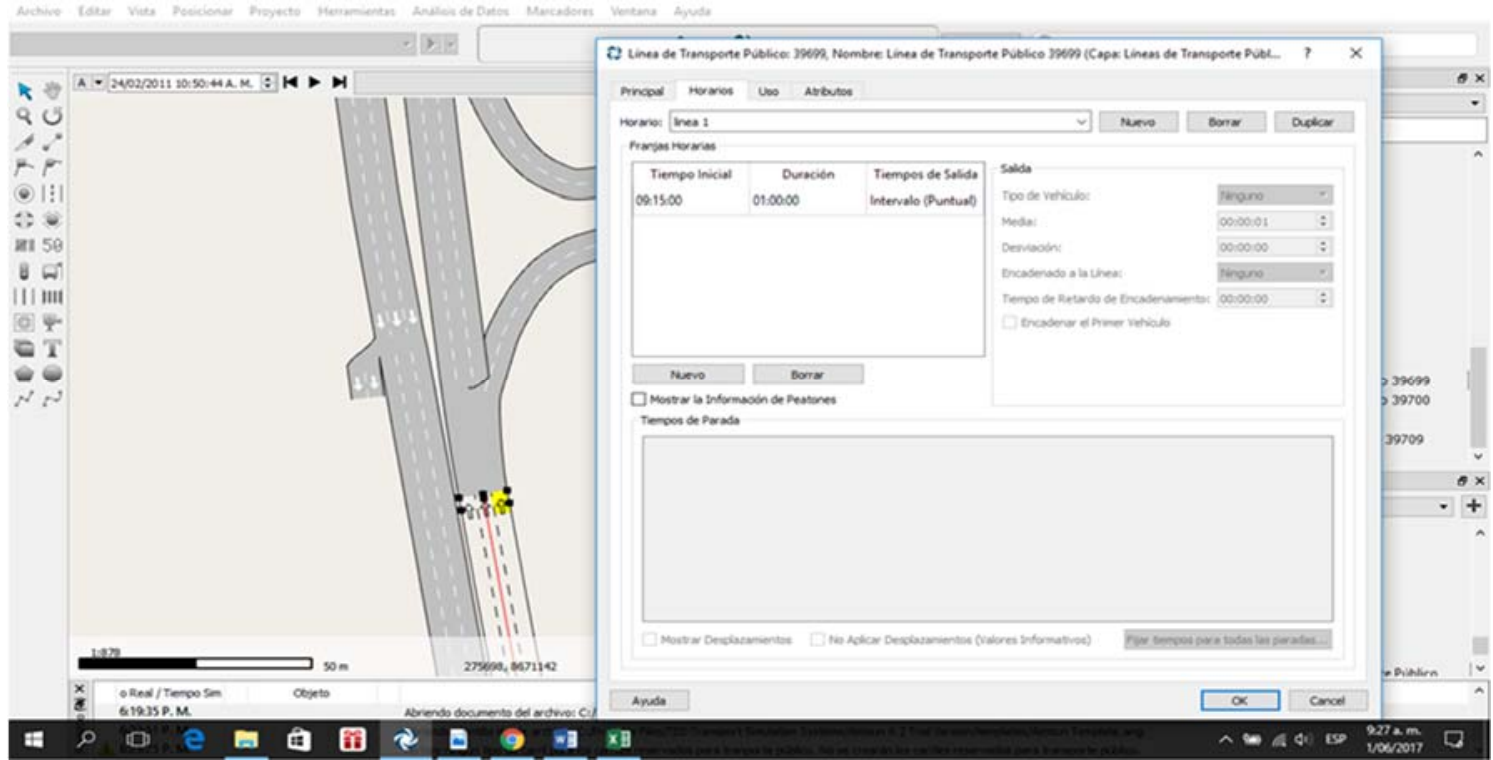

\subsubsection{Plan de transporte público}

Para cargar a la red de modelación las líneas de transporte público, debemos crear un "Plan de Transporte Publico" con la siguiente ruta: Proyecto - Nuevo - Transporte Publico 
- Plan de Transporte público. Al generar el plan de transporte público se agrupan todas las líneas de transporte con el propósito de incluir la influencia del tráfico de buses, tal como se aprecia en la Imagen 52.

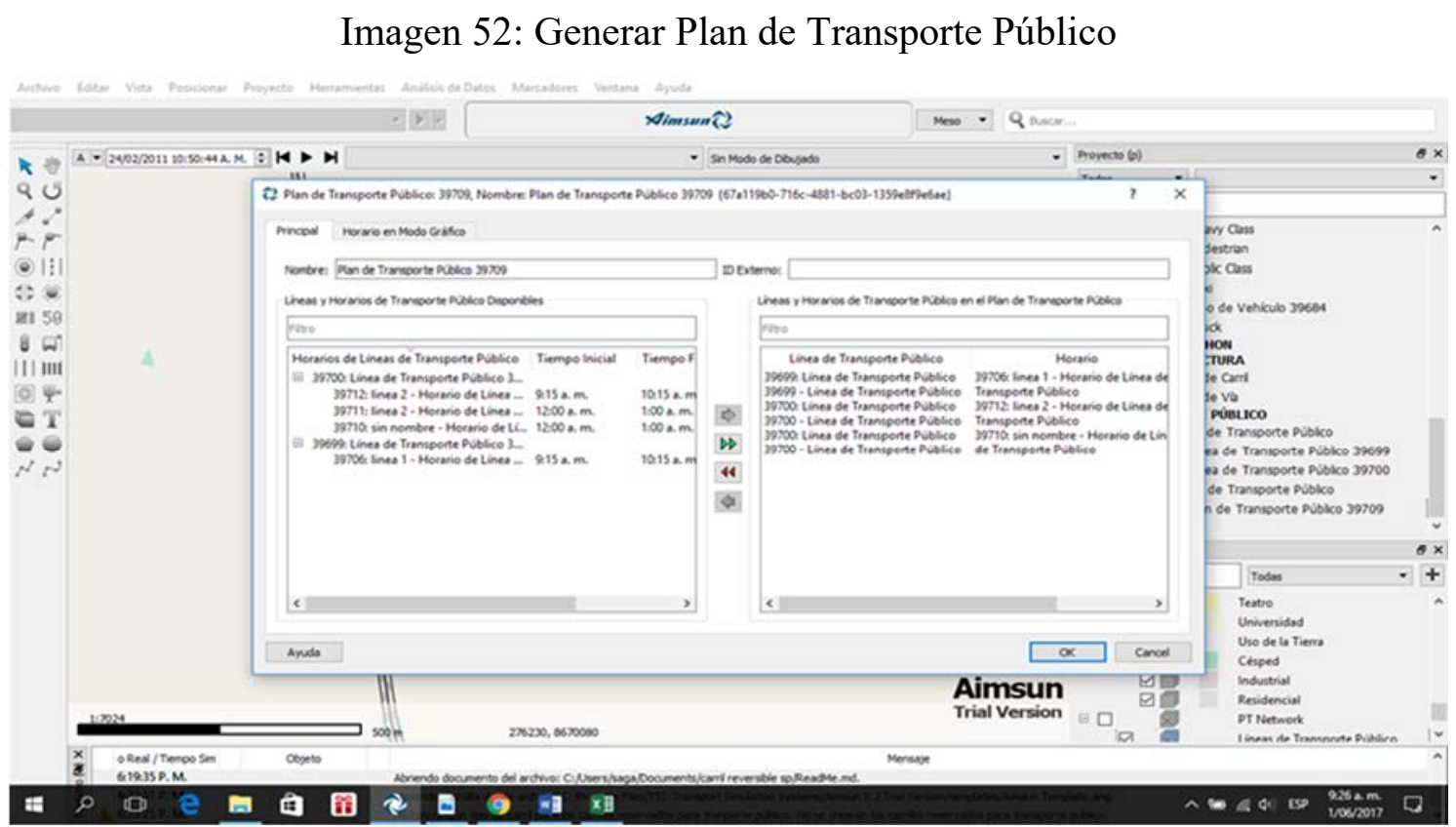

\subsubsection{Escenarios}

Una vez realizadas el ingreso de toda la información, se procede a realizar la modelación propiamente dicha, para ello es necesario crear el escenario a evaluar para estado de tráfico. Para realizar ello se tiene que primero crear el escenario según la siguiente ruta: proyecto-nuevo -escenario-escenario dinámico. El escenario se utiliza para proporcionar todos los datos de entradas y salidas realizadas referentes a la asignación de tráfico, transporte público, etc. Según la imagen 53 se ha realizado un escenario para el análisis mesoscópico con estados de tráfico. 


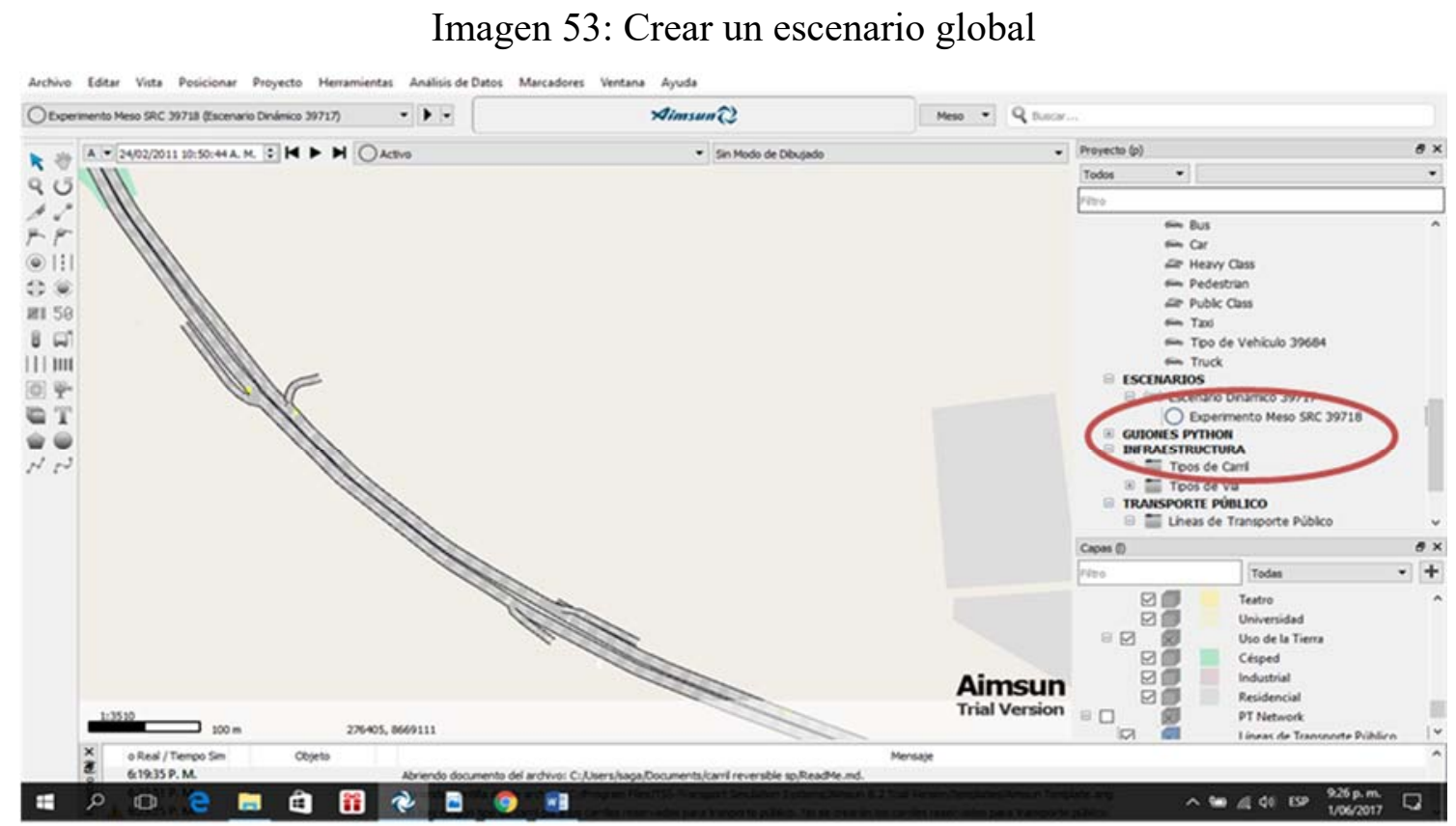

\subsubsection{Experimentos:}

Para establecer los distintos niveles de análisis, se tiene que generar un experimento en función al tipo de análisis a desarrollar, se selecciona con botón derecho sobre el escenario a analizar y se escoge "nuevo experimento".

\subsubsection{Análisis mesoscópico}

En este tipo de análisis las restricciones de aceleración y desaceleración no se usan y eso permite pasar de un modelo basado en un paso de simulación (microscópico) a un modelo basado en eventos ya que sólo se calculan los tiempos de entrada y salida de la sección.

La herramienta informática no muestra la simulación ya que utiliza datos generales y ofrece como resultado varios indicadores del estado de tráfico agregados según un intervalo de tiempo y tipo de vehículo, para todo el modelo, secciones, carriles, giros, sub-caminos y líneas de transporte público.

Para realizar el análisis, primero creamos un escenario mesoscópico, luego de escoger el tipo de experimento con "simulador mesoscópico", damos click en el experimento creado, y en la pestaña "Inicial” escogemos la opción "Estado inicial de simulación Usando calentamiento - Demanda de escenario - 10 minutos" 


\subsubsection{Número de Replicaciones}

Realizado lo anterior ahora hay que generar una replicación o un conjunto de replicaciones y calcular la media. Cada replicación es aleatoria y diferente por lo que produce resultados diferentes.

Para crear las replicaciones pulsamos el botón derecho sobre el experimento y seleccionamos "Nuevo - replicación" y en número de replicaciones.

Para correr la simulación, debemos dar click en el botón derecho y seleccionar: "Ejecutar Simulación en batch"

Imagen 54: Replicaciones en el caso de estudio

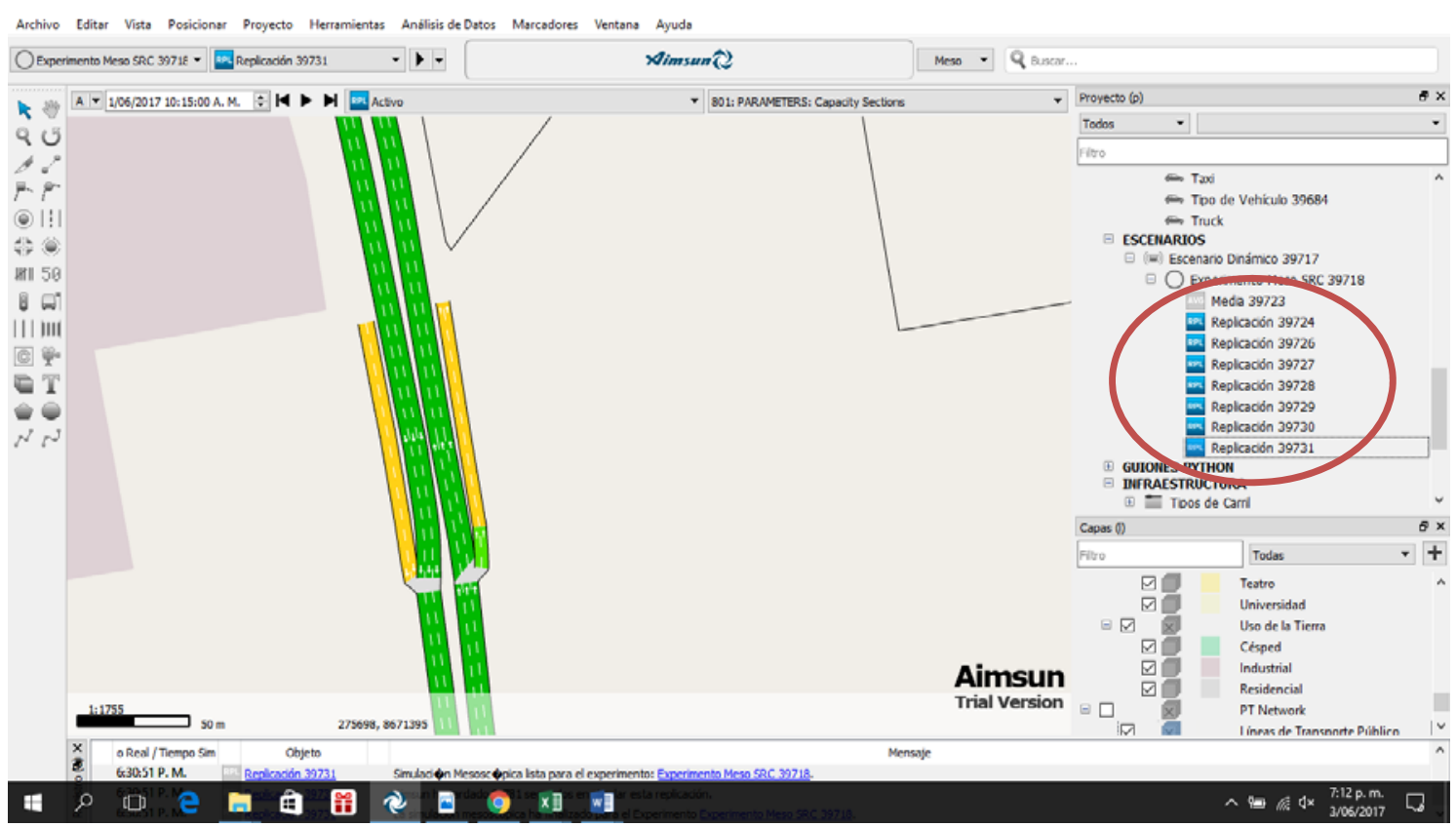

\subsubsection{Datos Obtenidos}

Los resultados de la simulación mesoscópica se detallan a continuación en el siguiente Imagen 55. Como se puede apreciar, el tramo Norte-Sur presenta una densidad de 63.32 $\mathrm{veh} / \mathrm{km} / \mathrm{c}$, lo cual implica que estamos a un nivel de servicio $\mathrm{F}$, mientras que en el tramo Sur-Norte( Ver imagen 56), la densidad llega a $16.09 \mathrm{veh} / \mathrm{km} / \mathrm{c}$ obteniendo un nivel de servicio C. 
Imagen 55: Datos Obtenidos Tramo Sur - Norte

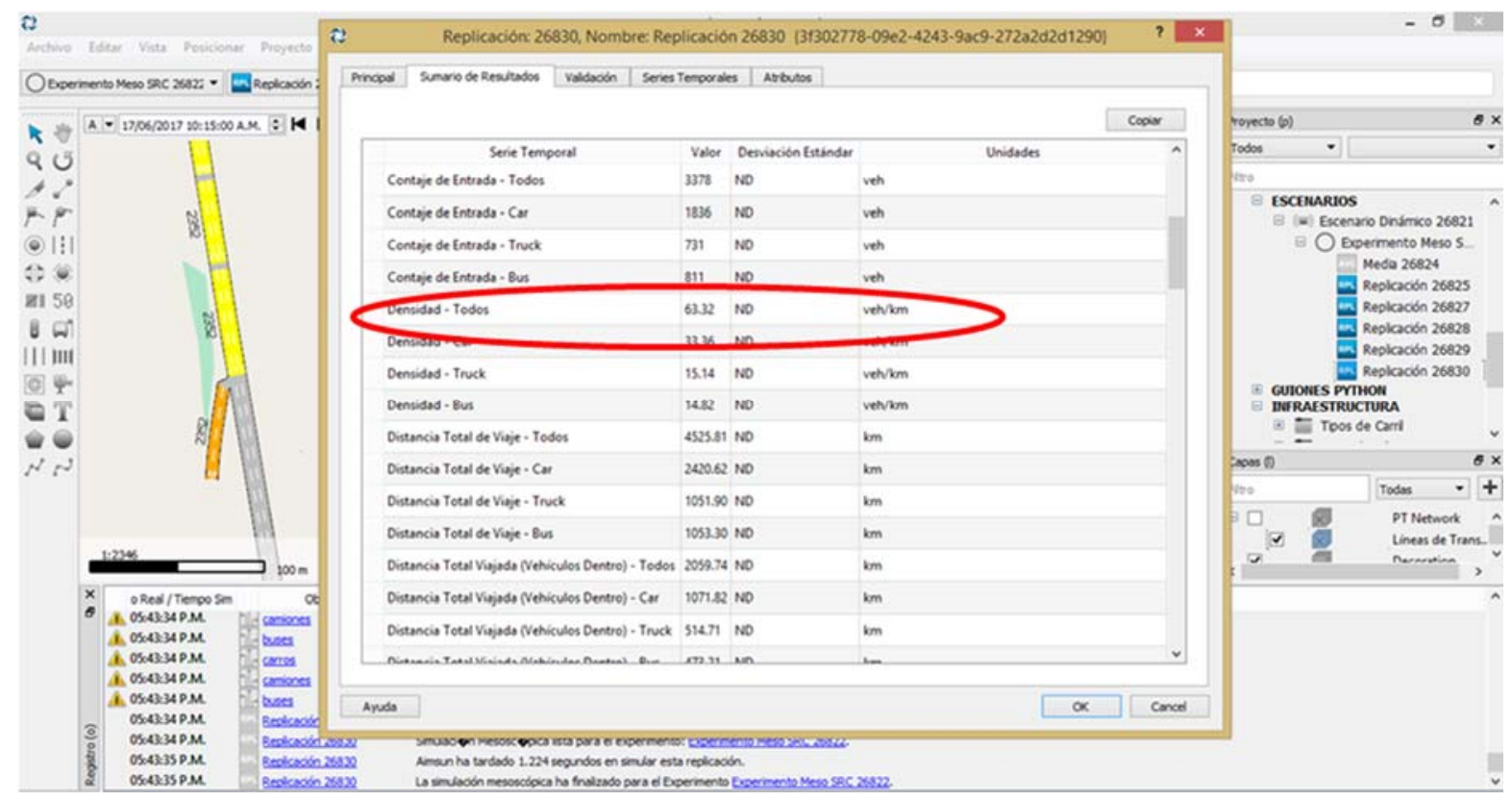

Imagen 56: Datos obtenidos en el Tramo Norte - Sur

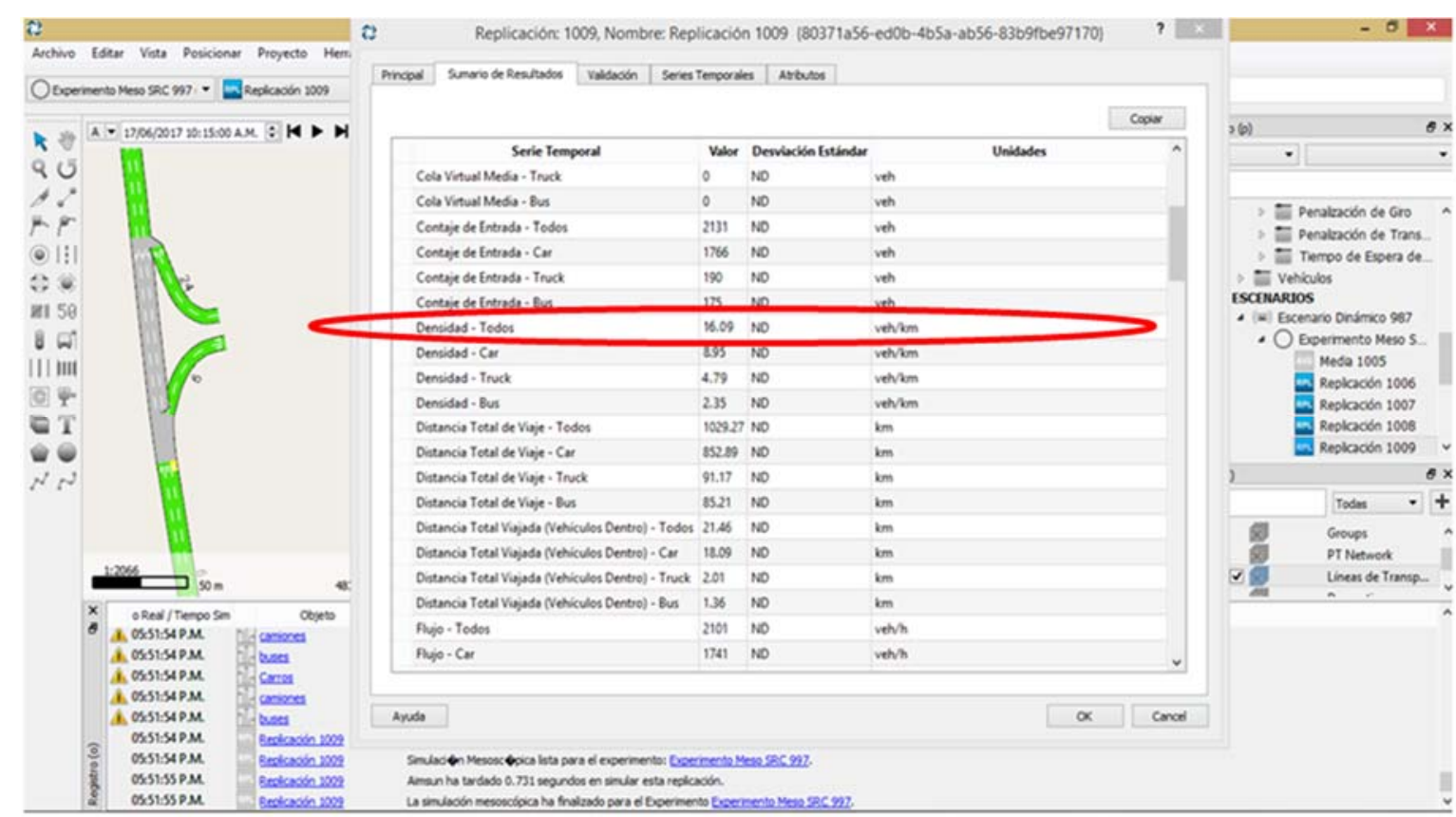

\subsection{ANALISIS Y COMPARACION DE LOS RESULTADOS CON LOS DATOS DE CAMPO:}

La herramienta permite analizar las variaciones de todos los parámetros de salida (velocidad, flujo, densidad, demoras, etc) dentro de la hora de demanda establecida, por ejemplo, para el análisis mesoscópico podemos ver como varia la densidad y el flujo 
dentro de la hora de máxima demanda dependiendo de los aforos vehiculares tomados en el caso de estudio y aplicando la gestión de reversibilidad en el tramo más congestionado.

Una vez obtenido los datos actuales del caso de estudio, se procede a rediseñar las rutas de tal forma que el tramo Norte - Sur presente 4 carriles y en el tramo Sur-Norte presente 2 carriles. En las Imágenes 57 y 58 podemos apreciar las rutas de nuestro caso de estudio diseñado en el programa Aimsun V8, en las que una vez rediseñadas para la gestión de reversibilidad tenemos resultados de densidades totales de $25.52 \mathrm{veh} / \mathrm{km} / \mathrm{c}$ con un nivel de servicio E y $20.51 \mathrm{veh} / \mathrm{km} / \mathrm{c}$ con un nivel de servicio D respectivamente, demostrando así que una vez aplicada esta gestión de reversibilidad en ambos tramos exista un balance de densidades en ambas vías.

Imagen 57: Tramo Eduardo de Habich, diseñado con la gestión de reversibilidad

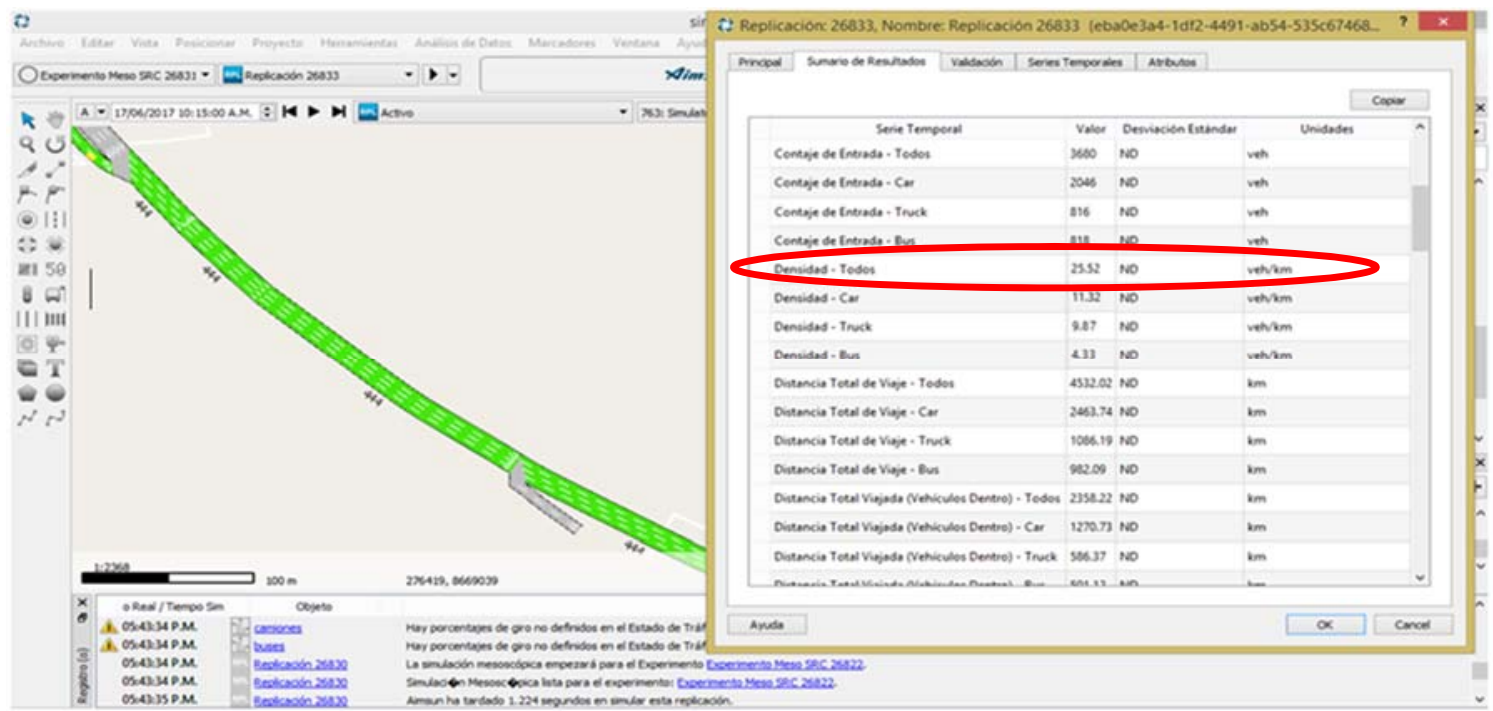


Imagen 58: Tramo cerca de Tomas Valle aplicando el diseño de reversibilidad.

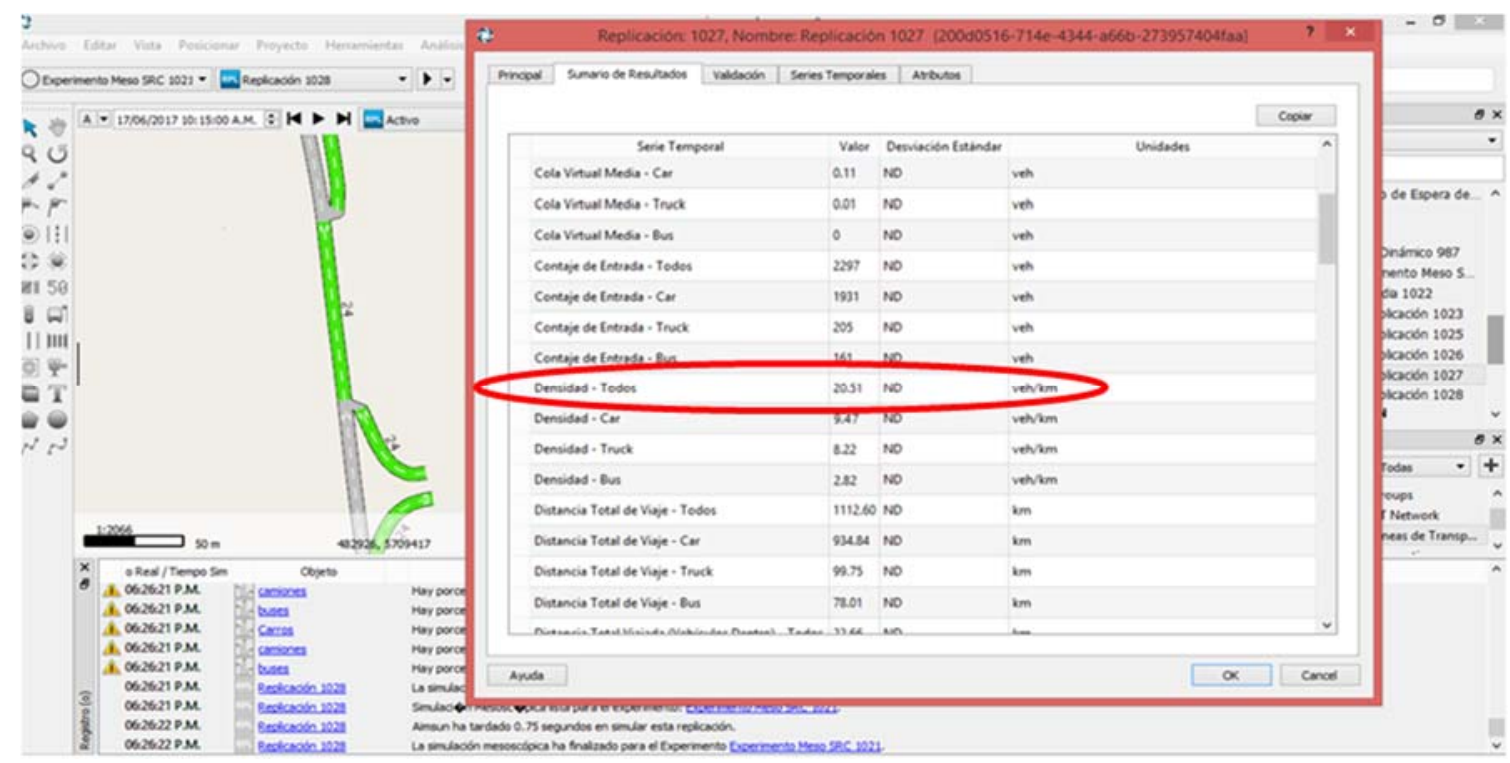

También podemos ver como varia la velocidad en la demanda analizada según las imágenes 59 y 60. En el tramo Sur - Norte se muestra que a medida que se aplica la gestión de reversibilidad, la densidad vehicular aumenta entre las (9:15 - 10:15am) y la velocidad empieza a disminuir, corroborando lo establecido en el concepto fundamental del tránsito.

Imagen 59: Tramo Norte - Sur con gestión de reversibilidad

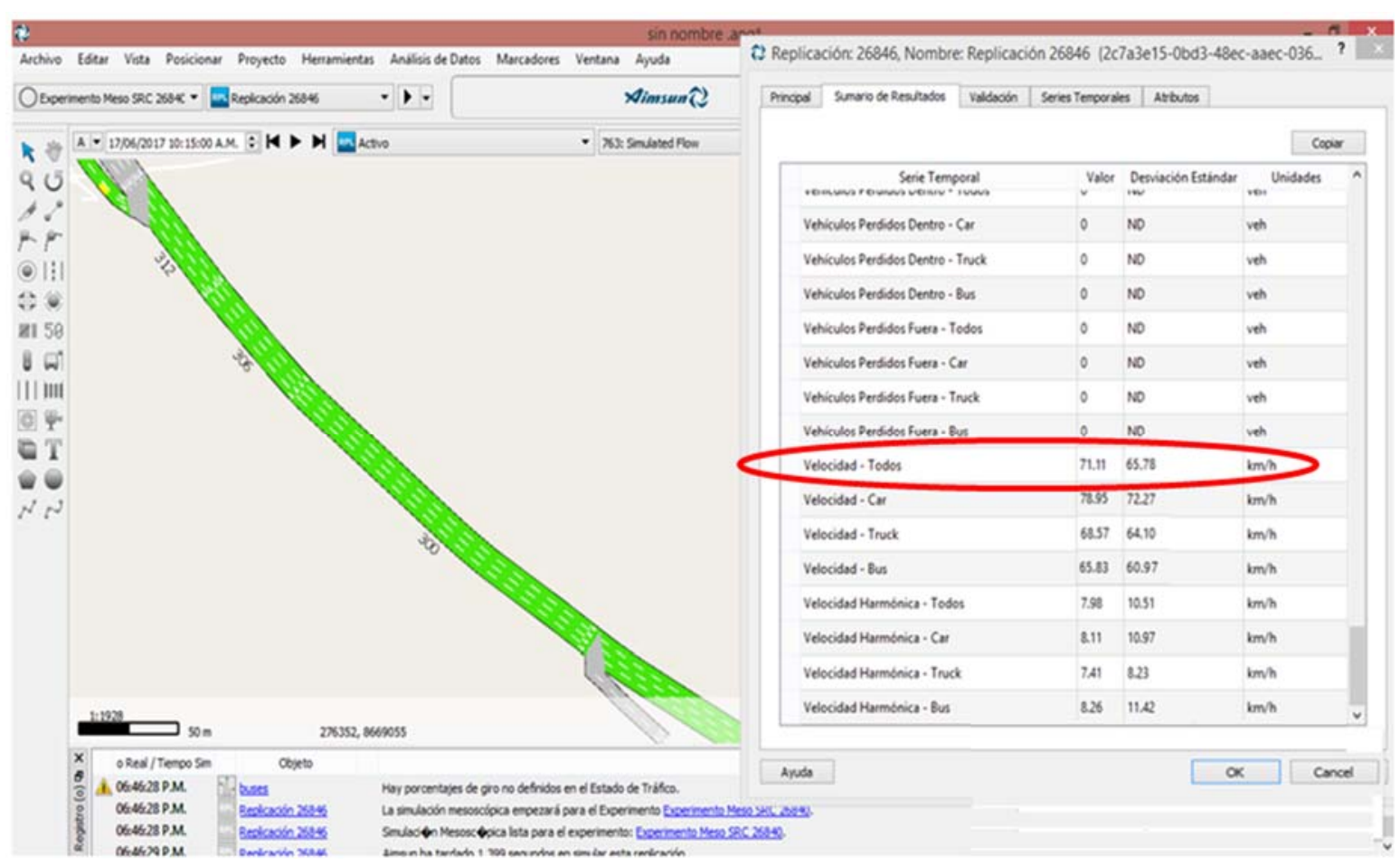


Imagen 60: Tramo Sur - Norte con gestión de reversibilidad

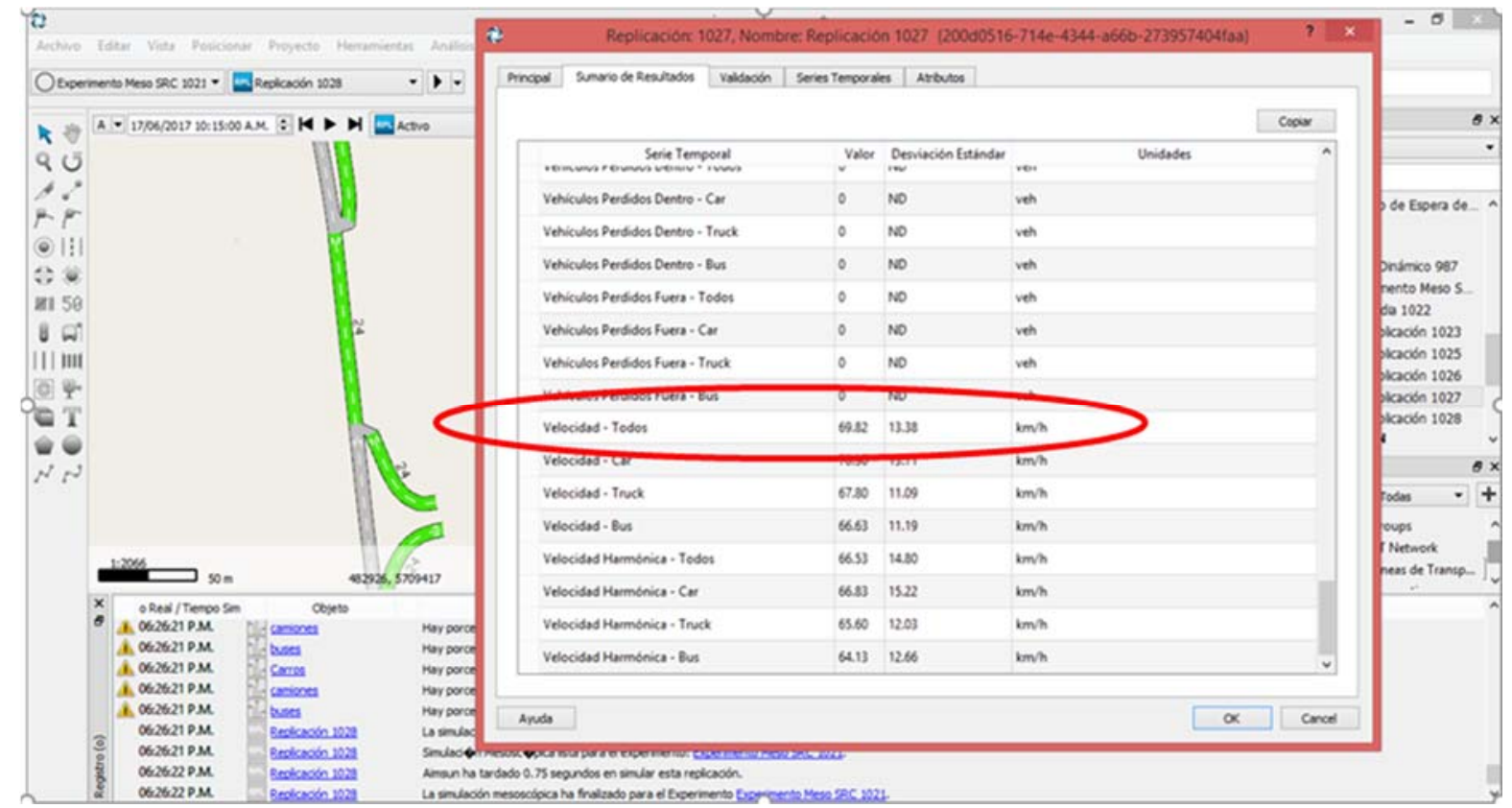

\subsection{CALIBRACION DEL MODELO CON DATOS DE CAMPO:}

Para poder calibrar los resultados obtenidos a través del software Aimsun V.8 se buscó un resultado que refleje la realidad con los datos obtenidos en campo, para ellos se compararon las densidades y velocidades.

A continuación se presentan los resultados obtenidos por el flujograma en el punto de control de aforo, en el puente control zarumilla. 
Imagen 61: Datos de Flujograma en un punto de aforo (Pte, Control Zarumilla)

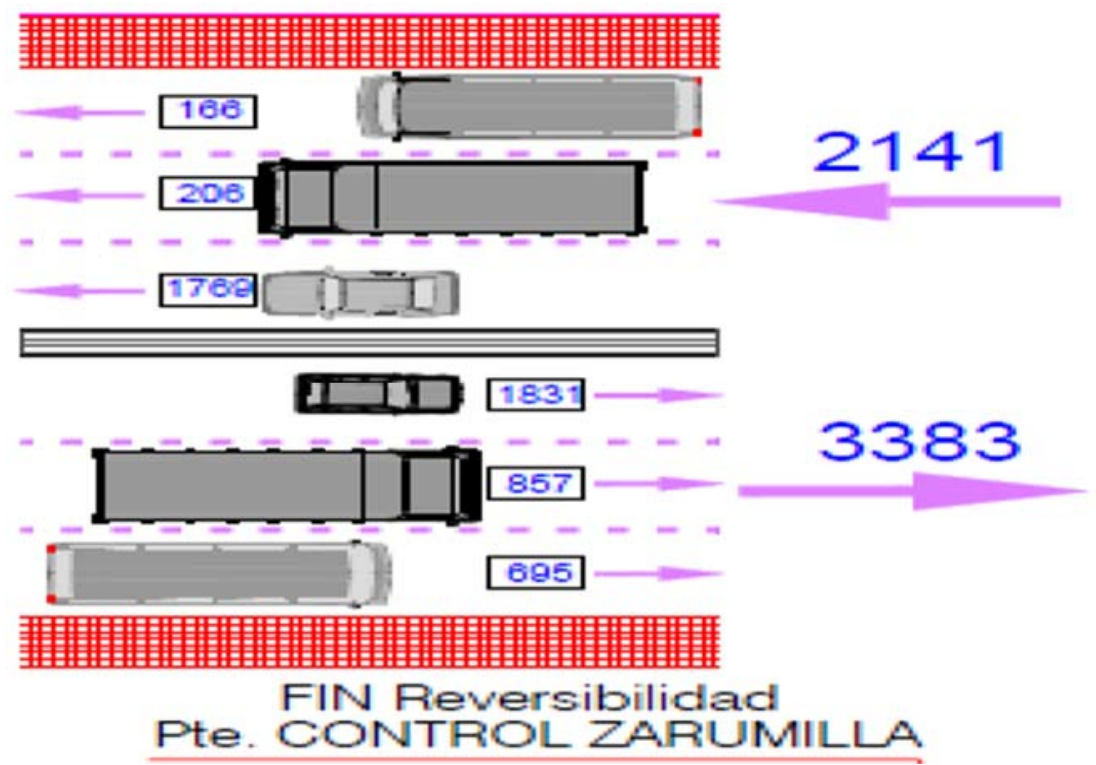

Posteriormente se presentan los resultados obtenidos en campo.

Imagen 62: Resultados de Campo (Pte, Control Zarumilla)

\begin{tabular}{|c|c|c|c|}
\hline \multirow{2}{*}{\multicolumn{2}{|c|}{$\frac{\text { TRAMO N-S }}{\text { Total de veh por } 3 \text { carriles } 3383 \text { Veh }}$}} & \multicolumn{2}{|c|}{ TRAMO S-N } \\
\hline & & Total de veh por 3 carrile & $2141 \mathrm{Veh}$ \\
\hline VMD por carril & $1128 \mathrm{Veh} / \mathrm{h}$ & VMD por carril & 714 Veh/h \\
\hline Distancia & $3.4 \mathrm{Km}$ & Distancia & $3.4 \mathrm{Km}$ \\
\hline Trecorrido & $0.2 \mathrm{~h}$ & T recorrido & $0.08 \mathrm{~h}$ \\
\hline V recorrida & $17 \mathrm{Km} / \mathrm{h}$ & V recorrida & $41 \mathrm{Km} / \mathrm{h}$ \\
\hline Densidad & $66 \mathrm{Veh} / \mathrm{h} / \mathrm{c}$ & Densidad & $17 \mathrm{Veh} / \mathrm{h} / \mathrm{c}$ \\
\hline
\end{tabular}

Se concluye con un comparativo entre el software Aimsun V8 con los datos obtenidos en campo para calibrar el modelo computacional, el cual valida resultados que se asemejen a la realidad. 
Imagen 63: Comparativo de datos (Pte, Control Zarumilla)

COMPARACION DE AIMSUN V8 CON DATOS DE CAMPO

\begin{tabular}{|lll|}
\hline DENSIDADES & & \\
\hline & Tramo N-S & Tramo S-N \\
AIMSUN V8 & $63 \mathrm{Veh} / \mathrm{h} / \mathrm{c}$ & $16 \mathrm{Veh} / \mathrm{h} / \mathrm{c}$ \\
DATOS DE CAMPO & $66 \mathrm{Veh} / \mathrm{h} / \mathrm{c}$ & $17 \mathrm{Veh} / \mathrm{h} / \mathrm{c}$ \\
\hline VELICIDADES & & \\
\hline & & \\
AIMSUN V8 & Tramo N-S & Tramo S-N \\
DATOS DE CAMPO & $20 \mathrm{Km} / \mathrm{h}$ & $50 \mathrm{Km} / \mathrm{h}$ \\
\hline
\end{tabular}




\section{CAPITULO VII: CONCLUSIONES Y RECOMENDACIONES}

\section{CONCLUSIONES:}

Nuestro caso de estudio se encuentra dentro de dos grandes proyectos hechos por la municipalidad de Lima, uno es el Intercambio Vial de Tomas Valle y el otro es el Intercambio Vial Eduardo de Habich, ambos con una gran inversión de 80 millones y 120 millones de soles respectivamente. Además, verificamos que en el caso de estudio existen tres tipos de vías como autopistas, carriles primarios y carriles secundarios. Todos estos tipos tienen como ancho de carril de 3.5m y las capacidades varían entre 2200, 1500 y $800 \mathrm{veh} / \mathrm{hr}$ respectivamente.

Se puede observar que el mayor congestionamiento vehicular se da en las mañanas, en el tramo de Norte a Sur, habiendo cerca de 7000 vehículos que pasan entre las 6am y $11 \mathrm{am}$, estos vehículos conforman un $88 \%$ de vehículos de 2 ejes y un $12 \%$ de vehículos de 3 o más ejes, mientras que en el tramo de Sur a Norte, pasan a esa misma hora un aproximado de 3000 vehículos siendo su composición un 95\% de vehículos de 2 ejes y un 5\% de vehículos de 3. Esto evidencia que el conjunto de la via del tramo de Norte a Sur se encuentre en un nivel de servicio F, mientras que en el tramo de Sur a Norte existe un nivel de servicio $\mathrm{C}$.

Con respecto a los resultados obtenidos en los distintos tramos con la ayuda del HCM 2010, se observa que la capacidad vehicular es superada por la demanda en el tramo Norte - Sur por las mañanas, ya que llega a un nivel de servicio F con unas densidades que superan los $100 \mathrm{veh} / \mathrm{mi} / \mathrm{c}(160 \mathrm{veh} / \mathrm{km} / \mathrm{c})$. Caso contrario pasa con el tramo de Sur a Norte, donde se tiene una densidad de $25 \mathrm{veh} / \mathrm{mi} / \mathrm{c}$ (40 veh/mi/c) dando un nivel de servicio C.

En la presente tesis se pudo evidenciar los puntos críticos donde se muestra una aglomeración de vehículos formándose nudos vehiculares en las ramales de entrada a la vía panamericana norte, en el tramo de Norte a Sur al término de intercambio vial tomas valle el cual se intercepta con los vehículos precedentes de la avenida Alfredo Mendiola y de la entrada de la avenida tomas valle, también se evidencio un nudo critico al termino 
del intercambio vial Eduardo de Habich la cual intercepta a los vehículos provenientes de la avenida Alfredo Mendiola. Por el contrario, en el tramo de Sur a Norte no se evidencia una gran aglomeración de vehículos.

Al aplicar el carril reversible los niveles de servicio del tramo más congestionado, que pasa de 3 a 4 carriles, disminuyen en un E, obteniendo densidades de $40 \mathrm{veh} / \mathrm{mi} / \mathrm{c}$ (64 $\mathrm{veh} / \mathrm{km} / \mathrm{c}$ ), mientras que el tramo que se queda con 2 carriles obtiene un nivel de servicio D con densidad de $32 \mathrm{veh} / \mathrm{mi} / \mathrm{c}(51 \mathrm{veh} / \mathrm{km} / \mathrm{c})$. Para verificar si esta gestión de reversibilidad es sostenible con el tiempo, se hizo una proyección de flujos vehiculares a 5 años para ver las nuevas densidades de los vehículos en el caso de estudio. En estos resultados se plasma en un $27 \%$ más de vehículos en ambos tramos, sobre todo en el del tramo Norte - Sur. Sin embargo, estos no pasan las densidades que se tiene actualmente sin aplicar ninguna gestión de reversibilidad.

Es evidente que el uso de la herramienta informática Aimsun 8.02, es sumamente versátil y útil al momento de realizar el trazado de la red y la carga de información de la misma, la cual se tiene que realizar con absoluto cuidado para que el modelo represente lo que ocurre en la realidad.

\section{RECOMENDACIONES:}

Se recomienda utilizar Aimsun v8 para futuros trabajos de gestión de reversibilidad, ya que en el presente trabajo ha contribuido para poder comparar los resultados del HCM 2010 a un nivel mesoscópico.

En cuanto a los datos obtenidos en campo, se recomienda continuar con el monitoreo de los aforos tomándolos toda la semana y con contadores mecánicos ubicados en puntos claves de la vía en estudio, actualizando los resultados de esta gestión para futuras mejoras.

Una vez implementado el carril reversible se recomienda tener en buen estado el funcionamiento de la vía, las señalizaciones y el buen reordenamiento del tráfico por parte de las autoridades correspondientes.

Se recomienda utilizar medidas de prevención para el diseño de los paraderos del caso de estudio, tal como se planteó en el puente Caqueta y la avenida Abancay las cuales se evidenciaba un caos vehicular, generándose nudos vehiculares. 
Se recomienda realizar un análisis comparativo de resultados, generando otras demandas de tráfico correspondientes a periodos y situaciones diferentes a la del presente documento ya que esto permitirá analizar mejor otras situaciones.

Se recomienda adecuar los parámetros del HCM 2010 a la realidad peruana, ya que hay factores de dicho manual que son implementados a una realidad americana, como es el caso del fp (Factor de comportamiento de conductor). Esto influencia mucho al momento de hallar los niveles de servicios, pues estos factores están relacionado directa e indirectamente al hallar las densidades.

Se pueden desarrollar modelos macro y micro utilizado la misma demanda y añadiendo más datos, lo cual se ahorraría tiempo en el proceso de ingreso de datos. 


\section{BIBLIOGRAFÍA}

BULL ALBERTO. (2003). Congestión de tránsito, El problema y como enfrentarlo. Santiago de Chile: CEPAL.

WALECZEK HELEN1, GEISTEFELDT JUSTIN2, (2016). Traffic Flow at a Freeway Work Zone with Reversible Median Lane. Transportation Research Procedia, 15, 257266. 25 de Agosto 2016, De ScienceDirect Base de datos.

MINISTERIO DE TRANSPORTES Y COMUNICACIONES: Manual de Dispositivos de Control del Tránsito Automotor para Calles y Carreteras - PERU (2000).

MINISTERIO DE TRANSPORTE Y COMUNICACIÓN. (Diciembre 2011). Perfil de tránsito en las principales vías del área metropolitana de Lima y Callao: Flujos Vehiculares (Años 2004- 2009 - 2011) Secretaría Técnica del Consejo de Transporte de Lima y Callao, 11, 1 - 24.

GABINETE DE ESTUDIOS TÉCNICOS INGENIERIA S.A. (2003). Estudios Técnicos y Ambientales del Corredor Segregado de Alta Capacidad (COSAC I) y sus terminales de transferencia. Lima - Perú: Grupo Consultor TarYet.

SIGMA GP - LOGIT.(2010). "Estudio del impacto vial en la red metropolitana de Lima y Callao por el flujo de carga del puerto, aeropuerto y zona de actividad logística". Lima - Perú: UT SIGMA GP-LOGIT.

SUB-COMITÉ DE INFRAESTRUCTURA. (2016). "Manual de dispositivos de control del tránsito automotor para calles y carreteras". Lima -Perú: Aprobado por la Resolución Ministerial No 210-2000-MTC /15.02.

ASOSIACIÓN TECNICA DE CARRETERAS. (2000). Manual de Capacidad de Carreteras, versión española de la Highway Capacity Manual. Madrid - España: Dirección General de Carreteras del MOPU.

SUB GERENCIA DE TRANSPORTE, LA MOLINA. (2011). Por tu tiempo...Soluciones Inteligentes. 28 de Agosto, de Municipalidad de la Molina Sitio web: http://www.munimolina.gob.pe/.

Cooperación Alemana, implementada por la Deutsche Gesellschaft für Internationale Zusammenarbeit (GIZ). (2016). Autoridad de Transporte Urbano para Lima y Callao: propuesta conceptual. 10 de Setiembre, de Ministerio de transportes y Comunicaciones de la República del Perú Sitio web: http://www.mtc.gob.pe/estadisticas/index. 
TRANSPORTATION RESEARCH BOARD. (2010). Highway Capacity Manual. Washington, DC: National Academies.

TRANSPORTATION RESEARCH BOARD EXECUTIVE COMMITEE. (2004). Convertible Roadways and Lanes. Washington: Academy of Sciences.

PEREYRA, GLADYS. (2016). By - pass de Habich: 8 años de congestión por mal diseño. 13 de Nobiembre 2016, de El Comercio Sitio web: http://elcomercio.pe/sociedad/lima/by-pass-habich-8-anos-congestion-mal-diseno-dronnoticia-1945735? flsm=1

MINISTERIO DE TRANSPORTES Y COMUNICACIONES . (Mayo, 2016). Manual de dispositivos de control del transito automotor para calles y carreteras. Lima: Dirección General de Caminos y Ferrocarriles.

CERQUERA, ESCOBAR, F. Á. (2007). CAPACIDAD Y NIVELES DE SERVICIO DE LA INFRAESTRUCTURA VIAL. Tunja: Banco de Objetos Institucional de la Universidad Pedagógica y Tecnológica de Colombia .

GALARRAGA, X. (1995). PEAJES URBANOS: Una reflexión actual sobre la congestión del tráfico en nuestras ciudades (Argumentos sociales, tecnológicos y económicos). Revista Internacional de los Estudios Vascos, 311.

TEJEDA, JULIO. Estudio de la capacidad de carreteras de dos carriles y su aplicación en Guatemala. Tesis Ingeniería Civil Guatemala, Universidad de San Carlos de Guatemala, Facultad de Ingeniería, 1975. pp. 1-69.

WILliaM R. McSHANE, ROGER P. ROESS, ELENA S. PRASSAS. Traffic Engineering. Segundo edición en idioma ingles, McGraw Hill 1999. pp. 206-216, 309333.

RADELAT G. (2003) Principios de Ingeniería de Tránsito, Washington D.C.: ITEInstitute of Transportation Engineers.

DAGANZO C. F. (1983) Derivation of Delays Based on Input-Output Analysis, Transpn Res. 17A, 341-342.

Arrieta, K. (2013). Modelación del tráfico vehicular con el software PTV Vissim -Tramo Bomba El gallo - Bomba El Amparo. Cartagena: Universidad de Cartagena

Aguirre, M. (2015). Sistemas, modelos y simulación [Fecha de consulta: 08 de Setiembre]. Recuperado de: http://arantxa.ii.uam.es/ aguirre/OS/sms.pdf Carvalho,dM.d(2015).dDiscrete and continuous simulation.[Diapositivas de power point].Recuperado de:http://es.slideshare.net/chienq1/discrete-and-continuoussimulationpresentation. 
Howard,dM. \& Karlin,dS.d(1998).kAn introduction to stochastic modeling (3rd edition). San Diego: Academic press.

Comite de Gestión Iniciativa Aire Limpio. (2010). II PLAN INTEGRAL DE SANEAMIENTO ATMOSFERICO PARA LIMA - CALLAO PISA 2011 - 2015. LIMA: Ministerio de Vivienda.

Geistefeldt, Justin. (2016). Assessment of Basic Freeway Segments in the German Highway Capacity Manual HBS 2015 and Beyond. Transportation Research Procedia, 15, 417-425. 27 de mayo del 2017, De ScienceDirect Base de datos.

Ning, Wu. (2013). A Stochastic Model for Reliability Analysis in Freeway Networks. 13th COTA International Conference of Transportation Professionals, 96, 2823 - 2834. 28 de mayo del 2017, De Procedia - Social and Behavioral Sciences Base de datos.

Chen, Ju; Wang Hao. (2013). Study on Flow Direction Changing Method of Reversible Lanes on Urban Arterial Roadways in China. 13th COTA International Conference of Transportation Professionals, 96, 807 - 816. 25 de mayo del 2017, De Procedia - Social and Behavioral Sciences Base de datos.

Casas, Jordi . Alex Torday. (2011). The need to combine different traffic modelling levels for effectively tackling large-scale projects adding a hybrid meso/micro approach. 14th EWGT \& 26th MEC \& 1st RH, 20, 251-262. 22 de mayo del 2017, De Procedia Social and Behavioral Sciences Base de datos.

Moyano, Christian . (2014). GUIA DE USO DE AIMSUN 8.02 PARA ANALISIS

MICROSCOPICO, MESOSCOPICO, HIBRIDO Y MACROSCOPICO. cuenca: UNIVERSIDAD DEL AZUAY FACULTAD DE CIENCIA Y TECNOLOGIA INGENIERIA CIVIL Y GERENCIA EN CONSTRUCCIONES TRANSITO Y TRANSPORTE. 